University of Tennessee Health Science Center UTHSC Digital Commons

\title{
Humanized Chimeric Receptors in the Therapy of Multiple Sclerosis
}

Ioana Moisini

University of Tennessee Health Science Center

Follow this and additional works at: https://dc.uthsc.edu/dissertations

Part of the Immune System Diseases Commons, and the Medical Sciences Commons

\section{Recommended Citation}

Moisini, loana , "Humanized Chimeric Receptors in the Therapy of Multiple Sclerosis" (2007). Theses and Dissertations (ETD). Paper 184. http://dx.doi.org/10.21007/etd.cghs.2007.0215. 


\title{
Humanized Chimeric Receptors in the Therapy of Multiple Sclerosis
}

\author{
Abstract \\ The role of autoreactive, antigen-specific T-cells in the development of autoimmunity has long been \\ documented. T-cells expressing chimeric receptors are specifically redirected against such cells and have \\ been proven to suppress autoimmune encephalomyelitis, the murine model of multiple sclerosis. We here \\ demonstrate the ability of humanized chimeric receptors to suppress experimental autoimmune \\ encephalomyelitis (EAE) in a humanized mouse model by redirecting $T$ lymphocytes against autoreactive \\ T-cells. The receptors were synthesized by linking the 84-102 epitope of human myelin basic protein \\ (MBP) to the extracellular and transmembrane domains of the beta chain of human major \\ histocompatibility complex (MHC) class II molecule and the cytoplasmic zeta chain of T cell receptor and \\ pairing it to the alpha chain linked to zeta. $\mathrm{CD} 8^{+}$receptor-modified T-cells (RMTC) were able to recognize \\ the cognate TCR receptor of antigen-specific cells and induce cytokine secretion, proliferation, and \\ cytolysis upon engagement. Most importantly, the RMTC were able to specifically kill antigen-specific \\ cells both in vitro and in vivo and prevent EAE disease. We hypothesize that the humanized chimeric \\ receptors could be used as a therapeutic approach for multiple sclerosis in the future.

\section{Document Type} \\ Dissertation \\ Degree Name \\ Doctor of Philosophy (PhD) \\ Program \\ Pathology \\ Research Advisor \\ Terrence L. Geiger, MD, Ph.D.

\section{Keywords} \\ multiple sclerosis, autoimmunity, chimeric receptors, receptor-modified T-cells, experimental autoimmune \\ encephalomyelitis, major histocompatibility complex, zeta chain, human myelin basic protein

\section{Subject Categories} \\ Diseases | Immune System Diseases | Medical Sciences | Medicine and Health Sciences
}




\title{
HUMANIZED CHIMERIC RECEPTORS IN THE THERAPY OF MULTIPLE SCLEROSIS
}

\author{
A Dissertation \\ Presented for \\ The Graduate Studies Council \\ The University of Tennessee \\ Health Science Center
}

In Partial Fulfillment

Of the Requirements for the Degree

Doctor of Philosophy

From The University of Tennessee

\author{
By \\ Ioana Moisini \\ December 2007
}


Copyright (C 2007 by Ioana Moisini

All rights reserved 


\section{DEDICATION}

This dissertation is dedicated to my mom, Valeria Moisini, who gave me endless love and support, and was there for me, come rain or come shine. 


\section{ACKNOWLEDGEMENTS}

I would like to thank my mentor, Dr. Terrence Geiger, for the trust, support, guidance, and most of all the patience that he has shown during my scientific struggle. He always knew how to ask things without demanding them and how to soothe my scientific frustrations. His particular way of approaching people and situations in and out of work has reshaped my character and my career. He is not only a prodigious contributor in his field, but also a very caring and considerate person. I appreciate and will always be grateful for the chance he took when he picked me out of three other colleagues to be his first graduate student.

I am also extremely thankful to Drs. Lawrence Pfeffer and Edward Schneider. The first one changed my life when he sent me the acceptance letter and has been like a father figure to me ever since I came here. He and Dr. Schneider have been the most "pro-student" figures at the University of Tennessee, supporting me and always being by my side when I felt that maybe research was not my true calling. Both of them "have sent" me back to the bench and boosted my spirit when I needed it the most.

Drs. Michael Levin and Elisabeth Fitzpatrick gave me a helpful and well-timed hand when my committee panel was changed; they did not hesitate for a moment to jump in and catch up with my research.

I am thankful beyond words to Dr. Richard Cross. My whole project would have turned to ashes if it had not been for his help with cell sorting. But he did more than provide scientific help and advice; he was the dearest friend and the strongest shoulder I could ever ask for and lean on. 
Last, but definitely not least, I have to acknowledge my colleagues and friends. Dr. Alina Nico West, Cynthia Lancaster, Dr. Robert Borgon, Yu Fukuda, my closest friend Dr. Weili Sun, Dr. Jean-Hugues Parmentier, Dr. Noel Lenny, Dr. Rajshekhar Alli, Dr. Donald Yergeau, Dr. Michelle Hamlet, Dr. Jana Radin, Dr. Kerim Babaoglu and so many more who have been close to me and supported me throughout these years. Our St. Jude lunches, parties, "complaints sessions", lab frustrations and so on are the best memories that I will carry on with me. I cannot thank you all enough. 


\begin{abstract}
The role of autoreactive, antigen-specific T-cells in the development of autoimmunity has long been documented. T-cells expressing chimeric receptors are specifically redirected against such cells and have been proven to suppress autoimmune encephalomyelitis, the murine model of multiple sclerosis. We here demonstrate the ability of humanized chimeric receptors to suppress experimental autoimmune encephalomyelitis (EAE) in a humanized mouse model by redirecting T lymphocytes against autoreactive T-cells. The receptors were synthesized by linking the $84-102$ epitope of human myelin basic protein (MBP) to the extracellular and transmembrane domains of the beta chain of human major histocompatibility complex (MHC) class II molecule and the cytoplasmic zeta chain of T cell receptor and pairing it to the alpha chain linked to zeta. $\mathrm{CD}^{+}$receptor-modified T-cells (RMTC) were able to recognize the cognate TCR receptor of antigen-specific cells and induce cytokine secretion, proliferation, and cytolysis upon engagement. Most importantly, the RMTC were able to specifically kill antigen-specific cells both in vitro and in vivo and prevent EAE disease. We hypothesize that the humanized chimeric receptors could be used as a therapeutic approach for multiple sclerosis in the future.
\end{abstract}




\section{TABLE OF CONTENTS}

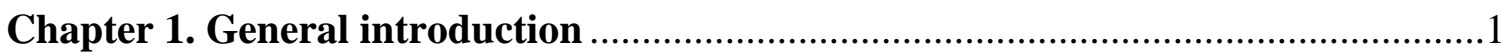

1.1 Historical perspective of multiple sclerosis ...............................................................

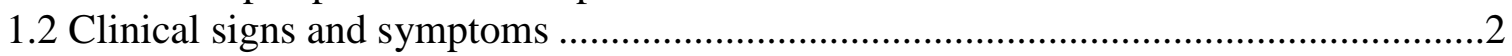

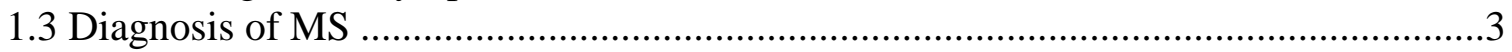

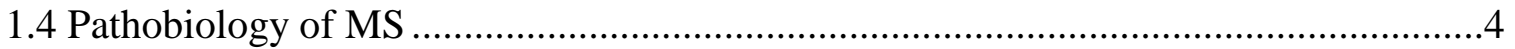

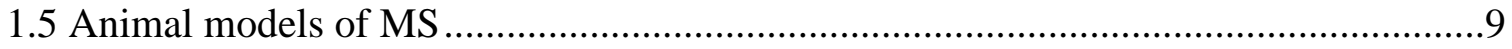

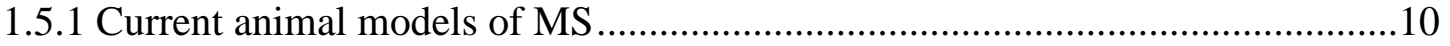

1.5.2 Adoptive transfer EAE................................................................................ 12

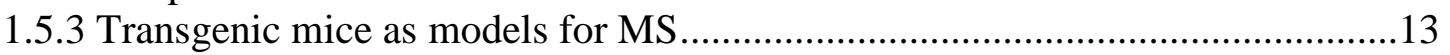

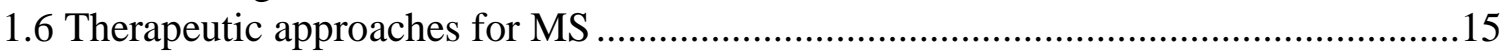

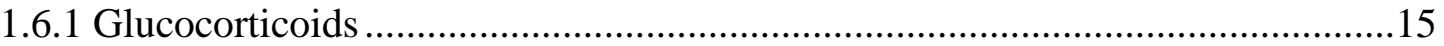

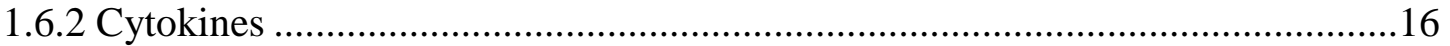

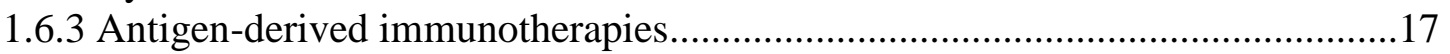

1.6.4 Altered peptide ligands .............................................................................. 18

1.6.5 Synthetic copolymers....................................................................................19

1.6.6 Mucosal administration of antigen............................................................20

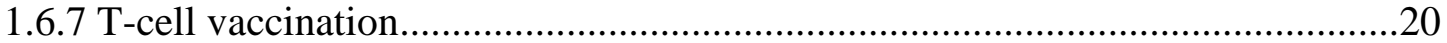

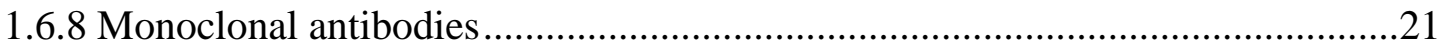

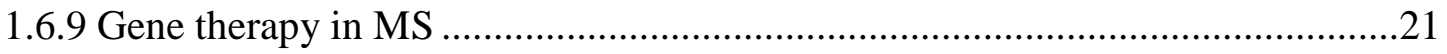

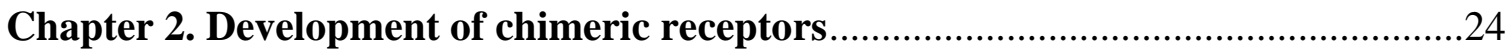

Chapter 3. Significance of a dileucine motif in CD28-zeta $(\zeta)$ containing chimeric receptors....................................................................................

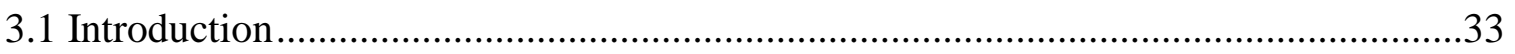

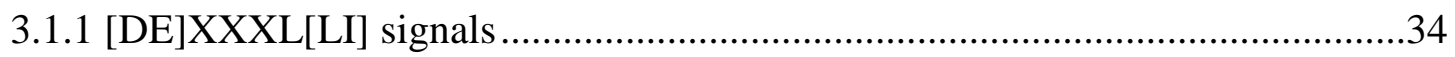

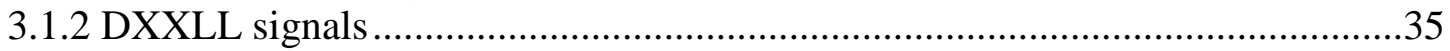

3.2 Identification of a murine CD28 dileucine motif that suppresses

single-chain chimeric T-cell receptor expression and function (1)..............................36

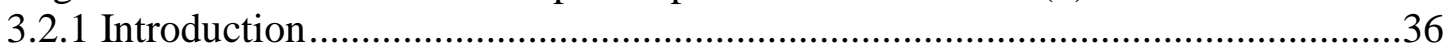

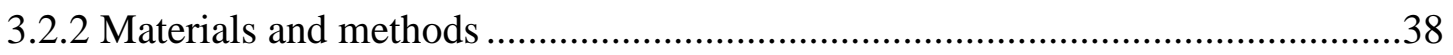

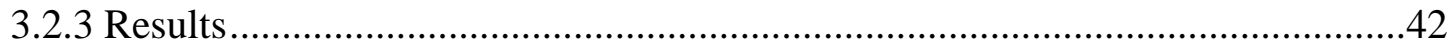

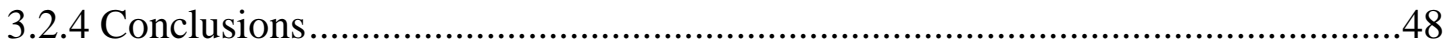




\section{Chapter 4. Development and function of humanized chimeric}

4.1 Introduction to the development of humanized chimeric receptors.............................53

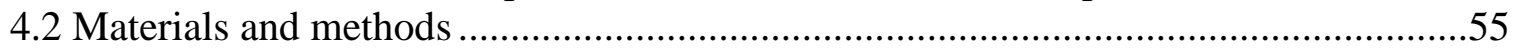

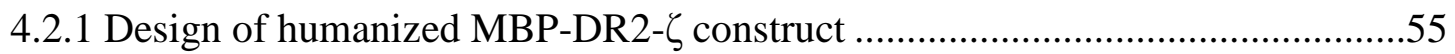

4.2.2 Design of humanized tailless MBP-DR2 construct ............................................65

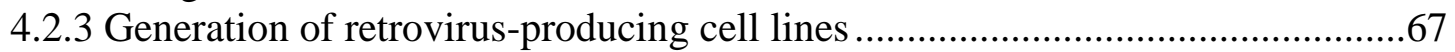

4.2.4 $\mathrm{Ob}$ and 6F11 target hybridoma cell lines ........................................................ 71

4.2.5 Stimulation of TCR/DR2 T lymphocytes …………......................................72

4.2.6 Stimulation of RMTC with HLA-DRB antibody ..............................................72

4.2.7 IL-2 secretion by RMTC in response to recognition of Ob hybridoma ……........................................................................................

4.2.8 IFN- $\gamma$ secretion by RMTC in response to recognition of $\mathrm{Ob}$

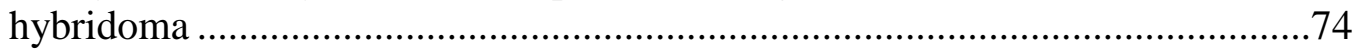

4.2.9 Proliferation of RMTC in response to stimulation by $\mathrm{Ob}$

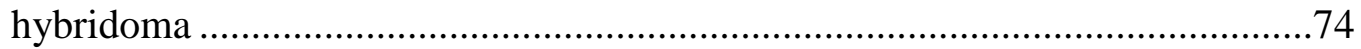

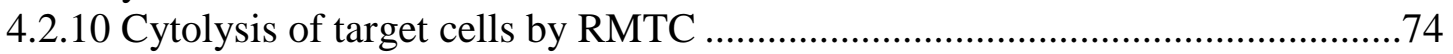

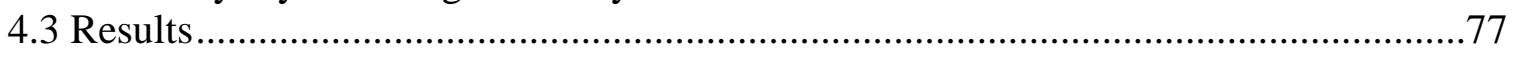

4.3.1 Design of chimeric receptors ...........................................................................77

4.3.2 Transfection of CRs into transient and permanent viral-

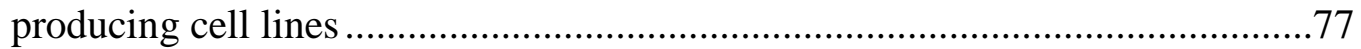

4.3.3 Transduction of 4G4 T-cell hybridoma and primary murine

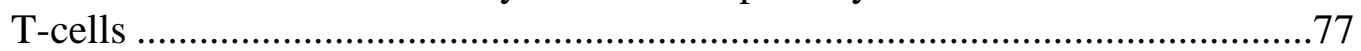

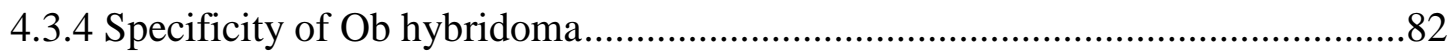

4.3.5 Specificity of T lymphocytes from TCR/DR2 transgenic mice...........................85

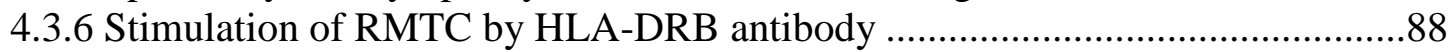

4.3.7 Specific recognition and stimulation of target cell lines by

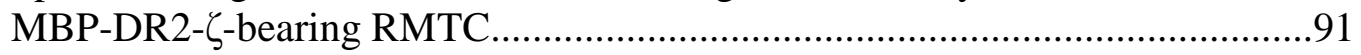

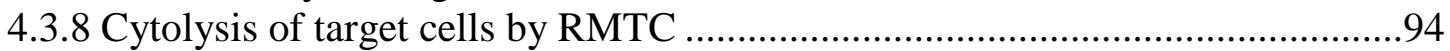

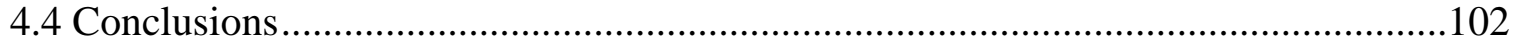

Chapter 5. In vivo activity of RMTC in humanized mice .........................................107

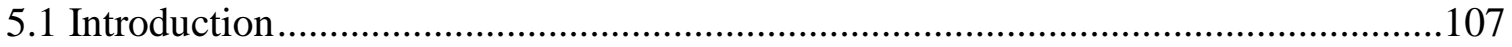

5.1.1 Humanized transgenic mice as MS models ......................................................107

5.1.2 Humanized HLA-DR2, TCR, and CD4 mice ………....................................108

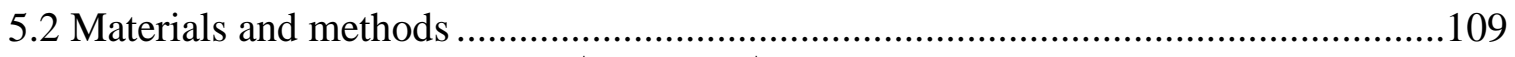

5.2.1 In vivo cytolysis of $\mathrm{CD}^{+}$and $\mathrm{CD} 8^{+} \mathrm{hMBP}$-specific cell lines

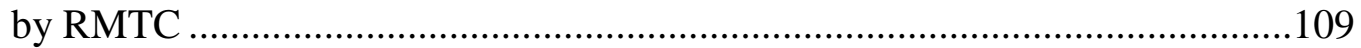

5.2.2 In vivo cytolysis of naïve antigen-specific T-cells by RMTC ...........................110

5.2.3 Prevention of EAE induced by naïve $\mathrm{CD} 4^{+} \mathrm{T}$-cells from TCR/DR2/hCD4 using RMTC .110

5.2.4 Prevention of hMBP84-102-induced EAE disease in TCR/DR2 double transgenic mice using therapeutic cells 


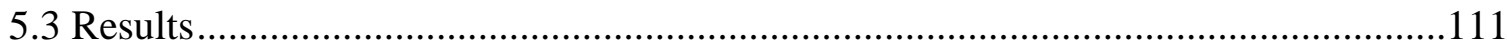

5.3.1 In vivo cytolysis of $\mathrm{CD}^{+}$and $\mathrm{CD} 8^{+} \mathrm{hMBP}$-specific cell lines

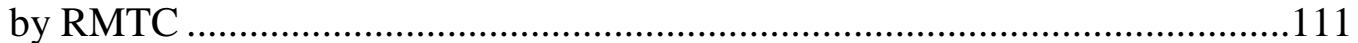

5.3.2 In vivo cytolysis of naïve antigen-specific T-cells by RMTC .........................113

5.3.3 Prevention of EAE induced by naïve $\mathrm{CD}^{+} \mathrm{T}$-cells from TCR/DR2/hCD4 using RMTC

5.3.4 Prevention of hMBP84-102-induced EAE disease in

TCR/DR2 double transgenic mice using therapeutic cells

5.4 Conclusions

Chapter 6. Discussion

.125

LIST OF REFERENCES 140

VITA 


\section{LIST OF TABLES}

Table 1-1. Clinical EAE scores ..................................................................... 11

Table 5-1. Clinical manifestations in $\mathrm{CD}^{+}$adoptive transfer EAE treated with RMTC

Table 5-2. Clinical manifestations in direct induction of EAE with hMBP and treatment with MSCV vector control or $\zeta$-bearing RMTC

Table 5-3. Clinical manifestations in direct induction of EAE with hMBP and treatment with MSCV control, $\zeta$-bearing, or tailless RMTC 


\section{LIST OF FIGURES}

Figure 1-1. H\&E staining of perivascular infiltrate in active MS plaque (top) and Prussian-blue staining of a MS plaque (bottom).

Figure 1-2. Immunopathogenesis of the MS lesion....................................................

Figure 2-1. Structure of the T-cell receptor and a chimeric receptor ...........................25

Figure 3-1. Chimeric receptor structure and sequence of the dileucine motif

Figure 3-2. Increased surface expression of dileucine-mutated chimeric receptor

Figure 3-3. Proliferative response of $\mathrm{K}^{\mathrm{b}}-\mathrm{CD} 28-\zeta$ and $\mathrm{K}^{\mathrm{b}}-\mathrm{CD} 28$

$[\mathrm{L} \rightarrow \mathrm{G}]-\zeta \mathrm{RMTC}$

Figure 3-4. IFN- $\gamma$ production by $\mathrm{K}^{\mathrm{b}}-\mathrm{CD} 28-\zeta$ and $\mathrm{K}^{\mathrm{b}}-\mathrm{CD} 28[\mathrm{~L} \rightarrow \mathrm{G}]-\xi$

RMTC

Figure 3-5. Cytolysis of antigen-specific T-cells by $\mathrm{K}^{\mathrm{b}}-\mathrm{CD} 28-\zeta$ and

$\mathrm{K}^{\mathrm{b}}-\mathrm{CD} 28[\mathrm{~L} \rightarrow \mathrm{G}]-\measuredangle \mathrm{RMTC}$

Figure 3-6. In vivo killing of antigen-specific T lymphocytes using

$\mathrm{K}^{\mathrm{b}}-\mathrm{CD} 28-\zeta$ and $\mathrm{K}^{\mathrm{b}}-\mathrm{CD} 28[\mathrm{~L} \rightarrow \mathrm{G}]-\xi \mathrm{RMTC}$

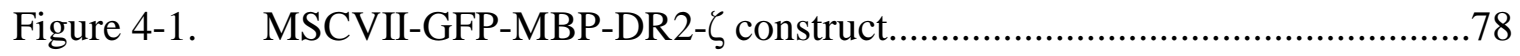

Figure 4-2. MSCV II-GFP-MBP-DR2 construct ………………………………....79

Figure 4-3. Transient retroviral transfection in 293T cells..........................................80

Figure 4-4. Retroviral transfection in GP+E86 cells .................................................

Figure 4-5. Expression of HLA-DR2 on 4G4 T-cell hybridoma

transduced with chimeric receptors

Figure 4-6. Expression of HLA-DR2 on primary murine CD8 ${ }^{+}$

T-cells transduced with chimeric receptors ...............................................84

Figure 4-7. Ob cells respond to stimulation by producing IL-2 …............................86 
Figure 4-8. Proliferation of TCR/DR2 cells in response to hMBP peptide.

Figure 4-9. $\quad \mathrm{CD}^{+}$and $\mathrm{CD} 8^{+} \mathrm{TCR} / \mathrm{DR} 2$ cells respond to stimulation by EL4-MBP-DR2

Figure 4-10. Stimulation of RMTC by anti-HLA-DR2 antibody .90

Figure 4-11. Intracellular IL-2 production by 4G4-MBP-DR2- $\zeta$ cells

Figure 4-12. IFN- $\gamma$ secretion by MBP-DR2- $\zeta$ RMTC stimulated by

Ob hybridoma

Figure 4-13. Proliferation of $\mathrm{CD}^{+} \mathrm{MBP}-\mathrm{DR} 2-\zeta \mathrm{RMTC}$ in response to Ob hybridoma

Figure 4-14. Cytolysis of Ob hybridoma by $\mathrm{CD} 8^{+}-\mathrm{MBP}-\mathrm{DR} 2-\zeta$ RMTC

Figure 4-15. $\quad \mathrm{CD}^{+} \mathrm{RMTC}$ show limited cytolysis of naïve TCR/DR2 cells from double transgenic (DTG) mice .98

Figure 4-16. Cytolysis of primary $\mathrm{CD}^{+}$DTG target cells by RMTC measured by stimulation of residual MBP-DR2-specific T-cells .100

Figure 4-17. Cytolysis of primary $\mathrm{CD}^{+}$DTG target cells by RMTC measured by stimulation of residual MBP-DR2-specific T-cells

Figure 4-18. Cytolysis of CD4 ${ }^{+}$hMBP-specific cell lines by RMTC .103

Figure 4-19. Cytolysis of CD8 ${ }^{+}$hMBP-specific cell lines by RMTC 104

Figure 5-1. In vivo killing of $\mathrm{CD}^{+} \mathrm{hMBP}$-specific cell line by RMTC .

Figure 5-2. Peptide stimulation of T-cells from mice that received RMTC and naïve antigen-specific target cells 114

Figure 5-3. Prevention of $\mathrm{CD}^{+}{ }^{+}$-adoptive transfer EAE with RMTC .116

Figure 5-4. Prevention of EAE with $\zeta$-bearing RMTC. .118

Figure 5-5. Prevention of EAE with $\zeta$-bearing or tailless RMTC .121 


\section{LIST OF ABBREVIATIONS}

\begin{tabular}{|c|c|}
\hline AICD & Activation-induced cell death \\
\hline AIDS & Acquired immunodeficiency syndrome \\
\hline AP-1 & Activating protein-1 \\
\hline APCs & Antigen presenting cells \\
\hline APL & Altered peptide ligands \\
\hline BBB & Blood brain barrier \\
\hline BCR & B cell receptor \\
\hline BDNF & Brain-derived neurotrophic factor \\
\hline BFA & Brefeldin A \\
\hline $\mathrm{Bp}$ & Base pairs \\
\hline$\beta 2 \mathrm{~m}$ & Beta2-microglobulin \\
\hline $\mathrm{CaCl}_{2}$ & Calcium chloride \\
\hline cDNA & cDNA \\
\hline CFA & Complete Freund's adjuvant \\
\hline CNS & Central nervous system \\
\hline ConA & Concanavalin A \\
\hline $\mathrm{Cr}$ & Chromium \\
\hline CRs & Chimeric receptors \\
\hline CSF & Cerebrospinal fluid \\
\hline CTL & Cytotoxic T lymphocytes \\
\hline DMEM & Dulbecco's modified Eagle's medium \\
\hline DTG & Double transgenic \\
\hline EAE & Experimental autoimmune encephalomyelitis \\
\hline Fab & Fragments of antigen binding \\
\hline FACS & Fluorescence activated cell sorting \\
\hline $\mathrm{Fc}$ & Crystalizable fraction \\
\hline FCS & Fetal calf serum \\
\hline$\gamma$ & Gamma chain \\
\hline GFP & Green fluorescent protein \\
\hline HIV & Human immunodeficiency virus \\
\hline HLA & Human leukocyte antigen \\
\hline HPBS & Hepes Phosphate Buffered Saline \\
\hline HSV-1 & Herpes simplex virus type 1 \\
\hline HsvTK & Herpes simplex virus thymidine kinase gene \\
\hline ICAM & Intercellular adhesion molecules \\
\hline IFN- $\beta$ & Interferon beta \\
\hline IFN- $\gamma$ & Interferon gamma \\
\hline $\operatorname{Ig}$ & Immunoglobulin \\
\hline Ii & Invariant chain \\
\hline IL & Interleukin \\
\hline IRES & Internal ribosomal entry site \\
\hline ITAM & Immunoreceptor tyrosine-based activation mo \\
\hline
\end{tabular}




\begin{tabular}{|c|c|}
\hline $\mathrm{KO}$ & Knockout \\
\hline LCMV & Lymphocytic choriomeningitis virus \\
\hline LN & Lymph nodes \\
\hline MBP & Myelin basic protein \\
\hline MHC & Major histocompatibility complex \\
\hline MOG & Myelin oligodendrocyte glycoprotein \\
\hline MRI & Magnetic resonance imaging \\
\hline mRNA & Messenger RNA \\
\hline MS & Multiple sclerosis \\
\hline MSCV & Murine stem cell virus \\
\hline $\mathrm{NaN}_{3}$ & Sodium azide \\
\hline NFAT & Nuclear factor of activated T-cells \\
\hline $\mathrm{NF}-\kappa \mathrm{B}$ & Nuclear factor-kappa \\
\hline OSE & Opticospinal EAE \\
\hline PBS & Phosphate buffered saline \\
\hline PCR & Polymerase chain reaction \\
\hline $\mathrm{PE}$ & Phycoerythrin-conjugated \\
\hline PLP & Proteolipid protein \\
\hline PPMS & Primary progressive multiple sclerosis \\
\hline PSG & Penicillin-streptomycin-glutamine \\
\hline PTx & Bordetella pertussis toxin \\
\hline $\operatorname{Rag} 2 \%$ & Recombination activating gene 2 knockout mice \\
\hline RMTC & Receptor-modified T-cells \\
\hline RRMS & Relapsing-remitting multiple sclerosis \\
\hline $\mathrm{scF}_{\mathrm{V}}$ & Single chain variable region \\
\hline Spl & Spleen \\
\hline SWB & Staining wash buffer \\
\hline TAA & Tumor associated antigens \\
\hline $\mathrm{TaV}$ & Thosea asigna virus \\
\hline TCR & T-cell receptor \\
\hline TCV & T-cell vaccination \\
\hline TGF- $\beta$ & Transforming growth factor-beta \\
\hline Th1 & T helper cells type 1 \\
\hline TNF- $\alpha$ & Tumor necrosis factor-alpha \\
\hline TTG & Triple transgenic \\
\hline VCAM & Vascular cell adhesion molecule \\
\hline $\mathrm{V}_{\mathrm{H}}$ & Variable region of the heavy chain \\
\hline $\mathrm{V}_{\mathrm{L}}$ & Variable region of the light chain \\
\hline ZAP-70 & $\zeta$-associated protein of $79 \mathrm{kDa}$ \\
\hline$\zeta$ & Zeta chain \\
\hline
\end{tabular}




\section{Chapter 1. General introduction}

\subsection{Historical perspective of multiple sclerosis}

In 1868, the French neurologist Jean-Martin Charcot examined a young woman who exhibited a new type of tremor, abnormal eye movements and slurred speech. On autopsy, she was found to have central nervous system (CNS) "plaques" that we now associate with multiple sclerosis (MS). Charcot named the disease "sclérose en plaques". By the end of the $19^{\text {th }}$ century, the major symptoms of MS were wellcharacterized and a new era of neurology arose.

Multiple sclerosis is a chronic progressive demyelinating disorder of the white matter of CNS characterized by loss of myelin with relative preservation of axons. It is the most common CNS autoimmune disease, affecting approximately 1 million people worldwide (250,000 in the United States) (2). The disease disproportionately affects females at a 2:1 sex ratio, has no single defined cause, and several genetic markers are associated with susceptibility.

Genes and environment play a major role in the pathology of MS. Among the primary genetic associations, human leukocyte antigen (HLA) class II genes on chromosome 6 and particularly HLA-DRB1 (HLA-DRB1*1501 and DQB1*0602) were found to increase the risk for MS (3). The risk of disease in a monozygotic twin of an affected individual is far greater than in dizygotic ones (25-30\% compared to $2-5 \%$ ). Also, the risk that first-degree relatives of patients with MS will get the same disease at some point in life is seven times higher than for the general population (4). These findings suggest a very strong genetic component (although not a Mendelian one). 
Epidemiological studies implicated geography as another factor for MS. Studies have shown that the disease is more prevalent in the temperate regions and western hemisphere (Germany, Scandinavia, Canada, northern US) and that migration from a high-incidence area to a low incidence one before puberty significantly decreases the risk of acquiring MS later in life (5). Therefore, environmental factors seemingly more prevalent in temperate regions combine with genetic factors to determine MS susceptibility.

\subsection{Clinical signs and symptoms of MS}

MS can exhibit a very large range of signs and symptoms commonly first seen in the third to the fourth decade of life. $85 \%$ of patients have the typical relapsing-remitting multiple sclerosis (RRMS), with episodes triggered by viral infections followed by remissions with no residual damage or different cumulative amounts of chronic impairment. Over time, approximately $30 \%$ of these patients will undergo transformation to a secondary progressive form with less frequent acute attacks, but with gradual worsening of symptoms and permanent disabilities. A subset of RRF is benign MS, with few and mild attacks and a limited disease course or total recovery. Ten percent of patients with MS develop progressive deterioration of neurological functions without relapses and a more aggressive form of disease, called primary progressive MS (PPMS) (6).

Symptoms displayed by MS patients vary according to the location of lesions. Cerebellar and cerebral plaques typically accompany speech and balance problems, tremors and loss of coordination. Motor and sensory nerve tracts deficits are revealed by 
spastic paralysis, muscle weakness, diplopia and other visual problems including blindness, urinary and bowel problems, and tingling, numbness, and loss of touch and pain. Patients may also show signs of depression, cognitive and emotional problems, fatigue, and sexual disturbances.

\subsection{Diagnosis of MS}

MS is not easy to diagnose. There is no single test adequate for diagnosis. Physicians rely on history, clinical signs and symptoms, and various tests including magnetic resonance imaging (MRI), cerebrospinal fluid (CSF) analysis, serology, and sensory evoked potential testing. The classic approach is manifestation of at least two clinical signs along with MRI lesions localized in the brain or spinal cord which confirm the diagnosis. MRI with gadolinium contrast shows enhancement of lesions that correlate with perivascular inflammation. Almost $90 \%$ of MS patients will show oligoclonal immunoglobulin (Ig) G in CSF. Although this finding is not specific for MS and can only be considered suggestive, it is very useful in ruling out infectious diseases or tumors that might mimic this autoimmune disease. Optic nerve lesions that might not show up on MRI can be detected by visual evoked potentials, which will reveal prolonged latencies consistent with plaques located within optic pathways. Serology is also not specifically a useful tool in diagnosing MS, but it proves helpful in differential diagnosis with other entities (7).

At the microscopic level, MS plaques reveal changes located primarily at the level of optic nerves, chiasm, tracts, brainstem, spinal cord, and cerebellum, consisting of perivascular edema and an inflammatory infiltrate of $\mathrm{T}$ lymphocytes and macrophages. 
Myelin is stripped from the axons impairing saltatory conduction and causing conduction block (Figure 1-1). Axons tend to be spared (8). During periods of remission, inflammation and edema subside and axons can undergo remyelination and carry out the normal function again. Repair of damaged areas occurs more completely in early stage disease when oligodendrocytes are still able to build a new myelin sheath; in time, as the disease progresses more advanced lesions develop characterized by gliosis. This creates a boundary between myelin producing cells and axons, therefore rendering remyelination inefficient.

\subsection{Pathobiology of MS}

There is no clear proof of the cause of MS. Epidemiological evidence from genetics, geography, and socio-economic factors has led many to hypothesize that there is a viral etiology (9). A definite pathogen has not been identified, though some microbes bear similar structures with self-antigens in the CNS such as myelin basic protein (MBP), myelin oligodendrocyte glycoprotein (MOG), and proteolipid protein (PLP) and it is possible that these promote autoimmunity through the mechanism of molecular mimicry. Molecular mimicry is the process by which a viral or bacterial infection causes activation of T-cells that are cross-reactive with self antigens. It is still a major mechanism for triggering autoimmune diseases (8).

There are other hypotheses for the etiology of MS including bystander activation and superantigenic $\mathrm{T}$ cell activation. In the bystander activation hypothesis, $\mathrm{T}$ lymphocytes are activated in the periphery by infectious agents presented on the surface of antigen presenting cells (APCs) and become capable of crossing the blood brain 

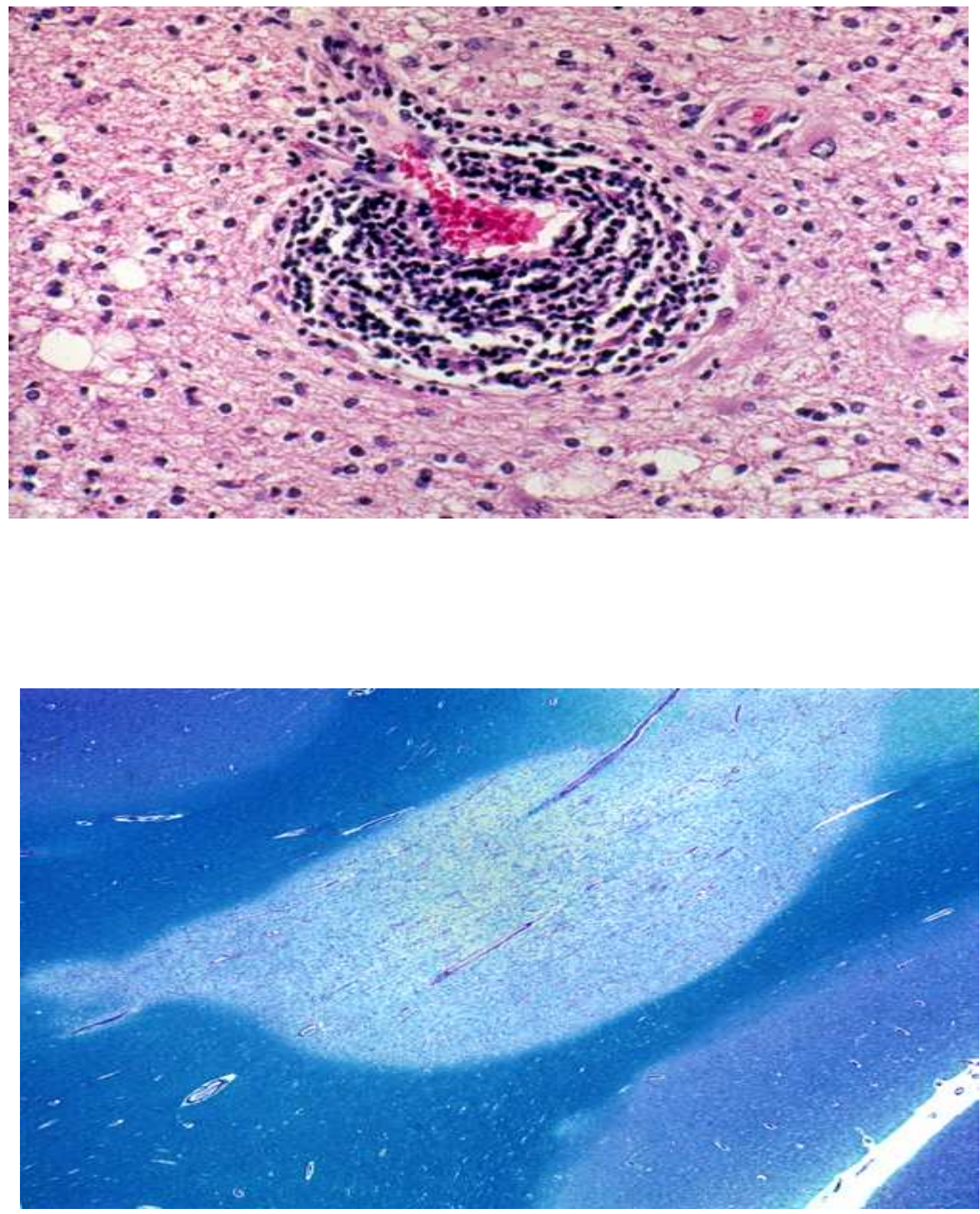

Figure 1-1. H\&E staining of perivascular infiltrate in active MS plaque (top) and Prussian-blue staining of a MS plaque (bottom). Source:

http://www.neuropathologyweb.org/chapter6/chapter6aMS.html (10). Accessed on January 21, 2007. 
barrier (BBB). The cells which develop into activated T-cells are $\mathrm{CD}^{+}$or so-called $\mathrm{T}$ helper cells type 1 (Th1). Although both Th1 and Th2 cells are present in MS, Th1 cells are able to secrete proinflammatory cytokines and also express a high level of adhesion molecules such as intercellular adhesion molecules (ICAM) or vascular cell adhesion molecule (VCAM). Inflammation upregulates adhesion molecule expression on the endothelium of the BBB, thus making it more permeable for penetration. Th1 cells also secrete matrix metalloproteinases that further compromise the integrity of the BBB (11). The opening of this natural barricade enables future inflammatory cells to penetrate into the CNS.

Once within the CNS, activated Th1 cells will die or be eliminated unless restimulated. An autoantigen or again, a microbe presented on the surface of CNS APCs (microglia) may restimulate and promote the expansion of T-cells, and induce the release of proinflammatory cytokines, such as interferon- $\gamma($ IFN- $\gamma)$ and tumor necrosis factor- $\alpha$ (TNF- $\alpha)$ that lead to macrophage activation. These cells release neurotoxic components (nitric oxide, reactive oxygen species) that damage the myelin sheath causing the structural and functional damage that results in MS (Figure 1-2).

In addition to this indirect means of tissue damage, infectious agents can directly damage tissues through recruitment of T-cells with new specificities, including autoaggressive T-cells to the CNS. In a process called epitope spread, the immune response can switch from being initially restricted to a microbial antigen to incorporate an added self antigen-specific response. Thereby, a secondary autoimmune reaction can develop (12).

Activation of T-cells by superantigens has also been proposed as a trigger for MS. 


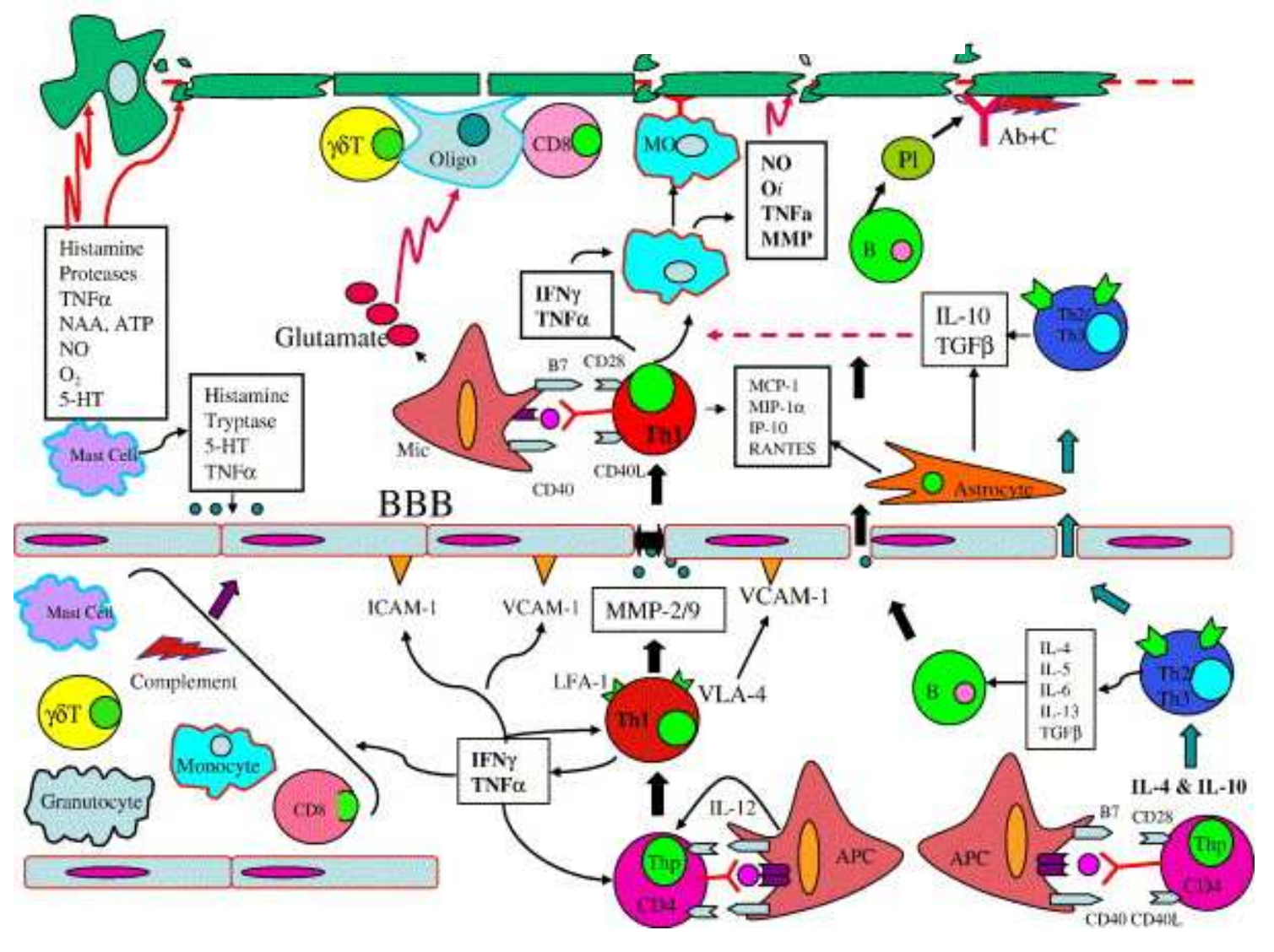

Figure 1-2. Immunopathogenesis of the MS lesion. APC = antigen presenting cell; IFN = interferon; IL = interleukin; Mic = microglia; $\mathrm{MMP}=$ matrix metalloproteinase; $\mathrm{MO}=$ monocyte $; \mathrm{NAA}=$ nacetylaspartate $\mathrm{NO}=$ nitric oxide $; \mathrm{Pl}=$ plasma;

VCAM = vascular cell adhesion molecule. Adapted and reprinted with permission Suhayl Dhib-Jalbut et al. 2006. Neurodegeneration and neuroprotection in multiple sclerosis and other neurodegenerative diseases. Journal of Neuroscience 176:198-215. 
According to this theory, virus and bacteria superantigen is able to cross-link the T-cell independent of peptide antigen, thus activating T-cells that can either expand or be deleted. Since superantigens preferentially recognize particular V $\beta$ families, a large proportion of T-cells might be activated during this process. As not all self-reactive Tcells are eliminated in the thymus by negative selection, myelin-specific ones can become activated and trigger an autoimmune response (13). Similar to the epitope spread theory, superantigen-mediated stimulation of autoreactive T-cells is detectable in mouse model but there is no direct evidence for this in MS.

For many years, Th1 $\mathrm{CD}^{+}$autoreactive T-cells have been incriminated as the major T cell offenders in MS. This theory has more recently been challenged by the recognition of a new subset of autoaggressive T-cells, $\mathrm{CD} 4^{+} \mathrm{Th}-17^{+}$, whose differentiation is promoted by APCs in the presence of interleukin (IL)-6 and transforming growth factor-beta (TGF- $\beta$ ) and whose expansion requires IL-23 (14). Studies show that Th17 cells secrete proinflammatory cytokines (IL-17, IL-6, TNF- $\alpha$ ), but not IFN- $\gamma$ and IL-4 and adoptive transfer of these cells can induce severe experimental allergic encephalomyelitis (EAE) disease (15). In models of MS and other autoimmune diseases, disease can be suppressed by blockade of IL-23 pathway or the downstream IL-17 and IL-6 factors (16).

$\mathrm{CD}^{+} \mathrm{T}$-cells are also incriminated for causing an immune attack by recruitment and clonal expansion within the CNS. They can recognize peptides presented by major histocompatibility complex (MHC) class I-expressing brain cells and even outnumber $\mathrm{CD}^{+} \mathrm{T}$-cells in the inflammatory infiltrate that characterize MS. Little is known about the role of CD8 cells in the pathology of MS in regards to the several aspects, such as 
means of CNS invasion, proliferation, apoptosis, and further clarifications are required (17). The invading cytotoxic T lymphocytes release pro-inflammatory cytokines, such as IFN- $\gamma$ and TNF- $\alpha$, thus inducing expression of MHC class I molecules in the brain in vitro (18).

It is clear that the above theories on the cause of MS are demonstrable in the mouse models but will be difficult to verify in humans. They leave many questions and lots of alternatives.

\subsection{Animal models of MS}

Modeling MS is a challenging task. The disease is complex and little is known about its triggers and mechanisms.

The first attempt to build an animal model of this disease was in the early 1930s when Rivers and collaborators noticed that certain infections (measles, smallpox) were followed by a wide range of CNS symptoms. Biopsy of these patients' brains revealed a perivascular demyelinating inflammatory infiltrate that characterized the acute disseminated encephalomyelitis. This group tried to reproduce the disease in monkeys by repeated intramuscular injections of extracts and emulsions of rabbit brain. Although the model did not accurately reproduce the human disease, it was still considered to be a groundbreaking discovery (19). A decade later, Kabat adjusted the disease-induction procedure by using an adjuvant to increase the immune response (20). This had two major consequences: it made the immunization protocol more manageable since the animals, unlike Rivers' model, only needed one injection, and it incriminated myelin as the culprit for MS since only animals injected with adult rabbit or monkey brain plus 
adjuvant got sick but not those injected with rabbit lung or fetal rabbit brain.

In 1960s and 1970s the concept of EAE developed as adoptive transfer of splenocytes from rats immunized with spinal cord extract into normal recipients was shown to induce disease (21).

\subsubsection{Current animal models of MS}

EAE is considered to be primarily mediated by MHC II-restricted CD4 ${ }^{+}$Th1 cells that secrete proinflammatory cytokines such as tumor TNF- $\alpha$ and IFN- $\gamma$ (22). EAE can be induced in susceptible strains of small, easy-to-handle animals such as mice, guinea pigs, and rats by active immunization with myelin antigens including MBP, PLP, MOG, and others, plus complete Freund's adjuvant (CFA). The administration of Bordetella pertussis toxin (PTx) increases the permeability of the BBB, thus creating a "breach" for activated T-cells that enter the CNS and cause the local inflammation (23). The first signs of neurological disease can be detected as early as ten days post immunization and depending on the model system may resemble the human relapsing-remitting or progressive disease forms. Inbred mice are most commonly used as the animal model for EAE due to their small size and well-defined genetics. Typically, disease is scored on a 1 to 5 scale (24) and the animals are euthanized at a score of 4 or 5 according to the ethical guidelines (Table 1-1).

Not all strains of mice exhibit the same disease course and symptoms when immunized with myelin antigens. While a certain strain of mice might be susceptible to a peptide antigen, another one could be resistant to the same antigen. There are now clear "recipes" for EAE induction in different mouse strains (25). Some of the standard 
Table 1-1. Clinical EAE scores.

\begin{tabular}{ll}
\hline Disease score & Clinical signs of disease \\
\hline 0 & No signs of disease \\
1 & Limp tail \\
3 & Partial hind leg paralysis \\
4 & Complete hind leg paralysis \\
5 & Hind and front leg paralysis \\
\hline
\end{tabular}




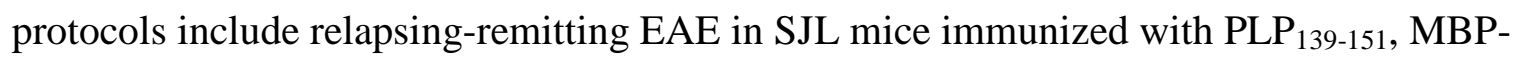
induced EAE in PL/J or B10.PL mice, or $\mathrm{MOG}_{35-55}$-induced EAE in $\mathrm{C} 57 \mathrm{BL} / 6$ mice. The disease is mostly T cell-mediated. However, MOG not only induces a T cell response, but also production of demyelinating autoantibodies (26). MOG-specific T-cells and autoantibodies were also found in circulation in patients with MS (27), but the cause that triggers their activation is still unknown.

\subsubsection{Adoptive transfer $E A E$}

The discovery that adoptive transfer of myelin-specific T-cells can induce EAE in naïve syngeneic recipients validated the autoimmune nature of EAE in mice (28).

Susceptible mice are immunized with a particular antigenic peptide followed by isolation of T-cells from draining lymph nodes and spleens, and in vitro stimulation of the T-cells with the myelin peptide. The T-cells are then injected into naïve recipients who develop disease. This approach showed that the CNS can be invaded by activated encephalitogenic $\mathrm{T}$ cell clones that are capable of crossing the BBB and emphasized the autoimmune nature of the phenomenon. The encephalitogenic cells were thought to bear a CD4 ${ }^{+} \mathrm{Th} 1$ cell phenotype and recognize self peptides presented in the context of $\mathrm{MHC}$ class II molecules. Later data showed that the $\mathrm{MOG}_{35-55}$ epitope is able to activate $\mathrm{CD} 8^{+}$ MOG-specific $\alpha \beta$ T cell receptor (TCR) positive cells that can be adoptively transferred into naïve C57BL/6 recipients, causing a much more severe clinical disease sustained by more destructive histopathologic CNS lesions. Furthermore, these antigen-specific cells were capable of surviving in vivo as shown by their ability to be retrieved from recipient mice (29). A role for $\mathrm{CD}^{+} \mathrm{T}$-cells in murine EAE has been controversial and not 
uniformly supported.

Although EAE induction seems fairly straightforward if following the rule "right

strain-right antigenic peptide", one must not overlook the association between the antigen and its corresponding MHC. T-cells cannot recognize an antigenic peptide properly unless it is presented on the right MHC. Beta2-microglobulin knockout $\left(\beta_{2} \mathrm{~m}^{-}{ }^{-}\right)$mice lacking MHC class I are resistant to EAE induced by adoptive transfer of $\mathrm{CD}^{+} \mathrm{MOG}-$ specific $\mathrm{TCR}^{+}$cells (29). Likewise, CIITA mice lacking MHC class II, as well as mice deficient for invariant chain (Ii) and H-2M (DM) are resistant to both direct priming with peptide and adoptive transfer of $\mathrm{CD}^{+}$peptide-specific T-cells. Interestingly, APCs from these knockout $(\mathrm{KO})$ mice can present MOG peptide to $\mathrm{CD}^{+} \mathrm{T}$-cells, but they are not capable of presenting and processing the myelin protein, thus rendering the mice resistant to EAE induction. The fact that MOG EAE cannot be induced in class II deficient mice but can in $\beta_{2} \mathrm{~m}^{-}$mice argues against a significant role for MOG-specific $\mathrm{CD}^{+} \mathrm{T}$-cells (30).

\subsubsection{Transgenic mice as models for MS}

Key to understanding human autoimmune diseases is to dissect the mechanisms of tolerance induction. The challenge for MS is to discover how seemingly tolerant selfreactive cells in the periphery become activated, are able to penetrate the CNS, and mediate disease. B10.PL mice were engineered to express a transgenic $\alpha 2^{+}$and V $\beta 8.2^{+}$ TCR specific for MBP epitope 1-11. These mice were highly susceptible to MBPinduced EAE and some even developed spontaneous disease. T-cells removed from spleens or lymph nodes of these animals proliferated and secreted cytokines in response 
to in vitro MBP stimulation (31). This MBP-specific transgenic mouse model has provided not only a unique model to study MS but also important information concerning the failure of the thymus to delete the antigen-specific T-cells, the mechanisms of peripheral tolerance, and the circumstances in which autoreactive, antigen-specific cells can cross the BBB and cause inflammation and myelin destruction (32).

Recently, another animal model has been developed that has proved extremely useful in the research of demyelinating diseases: the opticospinal EAE (OSE) mouse. A transgenic mouse expressing a TCR specific for MOG 35-55 (denoted TCR ${ }^{\mathrm{MOG}}$ ) was crossed with a MOG-specific Ig heavy-chain knock-in mouse (denoted $\operatorname{IgH}^{\mathrm{MOG}}$ ) both on a C57BL/6 background. The latter mouse had B cells producing antibodies against MOG. Single-transgenic mice did not undergo spontaneous EAE disease, but the doubletransgenic animals exhibited signs of an EAE-like disease, closer to the human Devic disease than to MS. Devic disease differs from MS only in regards to the site of the primary attack (spinal cord and optic tracts instead of the brain) and more frequent and severe attacks compared to MS. Nevertheless, mice exhibited a pathologic finding similar to what is seen in MS: inflammatory infiltrate with prevalence of $\mathrm{CD} 4^{+} \mathrm{T}$-cells and macrophage, demyelination, and sometimes axonal loss. Since single-transgenic mice did not develop spontaneous disease, one can infer that interaction between MOG-specific T and B cells present in the double-transgenic mice is responsible for development of disease. Although OSE mice do not reproduce the classical MS, they still are extremely valuable for the understanding of the role of B cells in the pathogenesis of EAE since MOG-specific B lymphocytes do not only act as APCs but also proficiently capture even smallest amounts of peptide, process it, and present it to T-cells (33). 


\subsection{Therapeutic approaches for MS}

The concept that MS is a non treatable disease has changed dramatically over time. There is currently no definitive cure, but long-term survival has increased in recent years due to new discoveries about the pathology of the disease, better and earlier diagnostics including MRI, and better clinical trial designs. While some therapies have been successfully introduced and have shown to improve symptoms, others, having been proven to work in animal models, have not shown any benefit or even worsened the disease course in humans. Nonetheless, efforts are increasingly centered towards making MS a much more manageable disease than in the past.

There are two goals to therapy: the first one is to treat disease symptoms, such as spasticity, vertigo, depression, bladder and bowel dysfunctions. The second one targets the pathogenic cascade: peripheral activation of antigen-specific T-cells, penetration of BBB and activation and proliferation in the CNS, demyelination, and interaction of TCR with peptides loaded on MHC complexes.

\subsubsection{Glucocorticoids}

Corticosteroids have long been considered a panacea for autoimmune conditions, and MS is no exception to the rule. Studies with intravenous (iv) methylprednisolone showed improvement of symptoms in patients with chronic progressive disease as well as acute relapses (34). Steroids have also proved beneficial for optic neuritis which is often the first clinical manifestation of MS (35). Therapeutic use of corticosteroids nevertheless has more recently decreased, partly due to their side effects (osteoporosis, glaucoma, worsened diabetes, suppression of adrenal glands, etc.), and partly due to the advent of 
new therapies. However, they are still important tools though in treating RRF of MS and secondary progressive MS.

\subsubsection{Cytokines}

Interferon beta (IFN- $\beta$ ) $1 \mathrm{a}$ and $1 \mathrm{~b}$ acts through a mechanism that is not fully understood, but it is proposed to involve a drop in IFN- $\gamma$ levels, blockade of myelin attack, inhibition of metalloproteinases, and various modulatory effects on chemokine and adhesion molecule production (36). Several drugs currently on the market (Avonex, Rebif, Betaseron) were shown to reduce the annualized relapse rate by approximately one-third. A new study revealed a decreased concentration of monocyte-derived nonclassical MHC molecule (class Ib) called HLA-G in patients with MS. This molecule is important since it inhibits both Th1 and Th2 cytokine production (IFN- $\gamma$, IL-2, and IL-10 respectively) by $\mathrm{CD}^{+}{ }^{+}$T-cells. HLA-G levels were increased to almost normal levels after treatment with IFN- $\beta$ (37).

TNF- $\alpha$ or cachectin has already been shown to be an important mediator in the pathology of MS due to its ability to mediate inflammatory responses. However, its therapeutic blockade in MS has been very controversial. Studies have shown that TNF- $\alpha$ is increased in the CSF of patients with MS in direct correlation to the severity of the disease and neurological impairment (38). However, results of anti-TNF- $\alpha$ therapy with TNF-receptor fusion proteins such as etanercept (Enbrel) or monoclonal antibodies such as infliximab (Remicade) have been disappointing. Disease course was worsened and there was actually a new case of MS reported (39). Data of TNF blockade in EAE has yielded confusing results. Treatment of adoptive transfer EAE with soluble TNF 
receptors reversed the disease and protected against recurrent episodes (40). In contrast, complete deletion of the gene in TNF knockout mice led to high mortality and severe neurological defects. Moreover, treatment with recombinant TNF reduced the disease course and even prevented progression of EAE (41).

IL-10 and TGF- $\beta 2$ are suppressive cytokines in EAE. IL-10 is produced by regulatory $\mathrm{CD}^{+}$cells and selectively upregulated during recovery in EAE model. Just like TNF- $\alpha$, studies of IL-10 treatment of EAE were mixed, particularly because the route of administration seemed to play an important role. Intravenous injections exacerbated the disease (42), whereas intranasal immunizations partially inhibited EAE (43). TGF- $\beta 2$ has not been approved for the therapy of MS due to the nephrotoxic effects seen in mice.

\subsubsection{Antigen-derived immunotherapies}

As corticosteroids and other drugs impair the general immune defense by eliminating or suppressing not only the disease-causing cells, but other T-cells, it has become imperative that therapeutic approaches specifically target antigen-specific cells. A major limitation in the therapy of MS is the phenomenon of epitope spread. This develops after the initiating event when CNS provides the proper environment for reactivation of T-cells (self-antigens and MHC complex and co-stimulatory signals necessary for reactivation), thus recruiting and stimulating cells reactive not only against the initial causative antigen, but also against other neighboring self-peptides. This leads to a more extensive repertoire of immune responses that may amplify tissue destruction. A major goal of antigen-derived therapies is to circumvent this problem by mediating bystander suppression. For example, self-reactive antigen-specific Th2 or Th3 cells that 
were initially generated by oral tolerization of mice and are capable of secreting antiinflammatory cytokines (IL-4, IL-10) may globally downregulate the immune response after activation (44). One method to generate these regulatory T-cells is through peptide tolerization. Two approaches to tolerization with peptides have been extensively studied: altered peptide ligands (APL) and mucosal administration of antigen.

\subsubsection{Altered peptide ligands}

It is well known that activation of $\mathrm{CD}^{+} \mathrm{Th} 1$ cells depend on the interaction of immunogenic peptide bound to MHC class II with the TCR along with a costimulatory signal from APCs. Activation leads to proliferation, cytokine production, and cytolysis. Lack of costimulation renders the cells anergic to subsequent antigenic stimulation. Past studies have shown that an immunogenic peptide that has some of its residues mutated can stimulate Th1 and Th2 cells to fulfill some functions, but not proliferation. This phenomenon is called partial activation. The basic principle is that the surface expression of important molecules, such as CD3 did not change, nor did the MHC binding residues in the peptide. The only residues that were changed were the TCR binding moieties. When the cells were cultured with the original peptide and APCs, the T-cells proliferated normally. When the cells were first stimulated with the mutated peptide, upon subsequent stimulation with the original peptide, T-cells were rendered unresponsive to the immunogenic peptide (45).

APLs were also approached in the therapy of EAE induced by adoptive transfer of a pathogenic $\mathrm{T}$ cell clone specific for MBP epitope $\mathrm{p} 87-99$. This clone caused a heterogenous inflammatory infiltrate of the CNS that disappeared when the clone was 
tolerized in vivo with an analogue of the immunogenic peptide that carried a phenylalanine to alanine substitution at position 96. Direct transfer of the same clone treated with the APL led to prevention of EAE and even reversion of paralysis. The mechanism of action is still unclear since the therapeutic APL has no influence on proliferation of pathogenic T cell clone to the immunogenic peptide MBP 87-99, so there is no MHC competition or TCR antagonism. It has though, been noticed that deletion of the inflammatory infiltrate by APL depends on the availability of IL-4. Treatment with APL promoted a shift in the ratio between IL- 4 and TNF- $\alpha$ to an increase in the former which downregulates the latter (46). Even though treatment of EAE with APL showed encouraging results, this approach in human MS has led to a controversial outcome, as some trials ending in exacerbations of disease (47).

\subsubsection{Synthetic copolymers}

Glatiramer acetate (Copaxone) consists of a mixture of alanine, glutamic acid, lysine, and tyrosine that acts like a universal antigen, "luring" autoreactive T-cells (48). It efficiently binds to MHC class II molecules (DR, but not DQ or class I), thus hindering self-peptide from binding in the same groove. It is not fully understood how the drug works: it was initially thought to cross-react with MBP peptide and then compete for MHC binding, but has also been found to induce regulatory T-cells (49). Since Glatiramer-activated, Th2-like cells can cross the BBB, they enter the CNS and secrete anti-inflammatory cytokines, such as IL-4, IL-10, IL-6, thus promoting a noninflammatory environment. These Glatiramer-activated cells also exert a neurotrophic effect by producing brain-derived neurotrophic factor (BDNF) (50). Copaxone can 
successfully prevent EAE induced by several peptides (MBP, PLP, or MOG) and phase III clinical trials have also shown it to be beneficial in RRMS (51).

\subsubsection{Mucosal administration of antigen}

Oral or nasal administration of antigens responsible for MS and EAE has yielded controversial results. Self-specific T-cells can be activated within six hours of oral administration of peptide and consecutive administration of the same peptide will decrease the number of effector T-cells. The route of administration is important, with the intranasal one seemingly more efficient than oral ("nasal" versus "oral tolerance"). Peptide administered intranasally seems to be able to reach the thymus where it can mediate apoptosis of high affinity thymocytes as opposed to the oral route of administration where the antigenic peptide might be destroyed by the acid in the gastrointestinal tract. The mechanisms governing mucosal tolerance - anergy or deletion of antigen-specific T-cells - are not clear. Although feeding the inducing peptide at time of disease induction prevented EAE in mice and generated enthusiasm regarding the therapeutic outcome of the human disease (37), this approach failed in MS clinical trials (52).

\subsubsection{T-cell vaccination}

Antigen-stimulated T-cells can induce EAE equally as well as the antigenic peptide itself in adjuvant. T-cell vaccination (TCV) uses irradiated, activated antigenspecific CD4 $4^{+}$T-cells to "vaccinate" mice without causing EAE similar to microbial vaccination against infectious agents. These cells induce $\mathrm{CD} 8^{+} \mathrm{T}$-cells capable of killing 
the autoreactive $\mathrm{CD} 4^{+} \mathrm{T}$-cells as well as preventing antigen-induced proliferation of the vaccine T-cells themselves. Pilot trials in MS patients have used TCV with MBP-reactive T-cells isolated from their blood, activated in vitro and irradiated to abrogate subsequent proliferation. These clones were then injected back into the patients, resulting in the specific deletion of circulatory MBP-specific T-cells (53). Although this procedure has not been used to treat patients on a large scale, it is still considered for future evaluation.

\subsubsection{Monoclonal antibodies}

Activated T-cells express high levels of surface adhesion molecules like VCAM or ICAM and upregulate their receptors on the endothelium of the BBB, thus enabling Tcells to cross the BBB and cause inflammation in the CNS. Antibodies directed against the ligand-receptor pair could potentially block this first step in the CNS homing of Tcells. VCAM-1 expression is low on blood vessels under homeostatic conditions; however, expression is increased under conditions of inflammation, such as found in brain tissue of EAE-induced animals and human MS. Administration of antibody against $\alpha 4 \beta 1$-integrin prevents accumulation of leukocytes in the brain and subsequent development of EAE (54). The monoclonal antibody anti- $\alpha 4 \beta 1$-integrin was named Natalizumab and has been licensed for clinical use (55).

\subsubsection{Gene therapy in MS}

Gene therapy for autoimmune disease has emerged as a result of progress in deciphering in greater detail the pathologic mechanisms by which self-antigens mediate autoimmune diseases. This approach can be very specific and aims to deliver a gene or 
gene product that can specifically block disease. Gene therapies may be antigen-specific, while hopefully avoiding the general suppression of the immune system associated with conventional treatments. The goal of gene therapy in MS is generally to deliver immunemodulating molecules (blocking antibodies, anti-inflammatory cytokines, etc.) by different means such that antigen-specific immune tolerance can be achieved.

One new tactic is the delivery of anti-inflammatory cytokine genes (IL-4, IL-10, or IL-12 p40 subunit) by viral vectors that are administered intrathecally in order to directly concentrate the gene product in the CNS, slowly releasing the cytokine of interest. Different vectors may be used: non-replicative herpes simplex virus type 1(HSV1), retroviruses or adenoviruses are able to accommodate the genes and infect cells. Potentially, EAE could be both prevented and treated using an HSV-IL-4 system by downregulating proinflammatory cytokines and therefore, macrophage activation and CNS invasion (56).

An even more practical way of delivering anti-inflammatory cytokines by means of viruses is to retrovirally transduce antigen-specific $\mathrm{CD} 4^{+} \mathrm{T}$-cells. The autoreactive $\mathrm{T}$ cells will migrate to the CNS and therefore, provide a "home delivery" of Th2 regulatory cytokines to the autoimmune lesions (57).

Receptor-modified T-cells (RMTC) have emerged in the past five years as a means to redirect T-cells against antigen-specific T-cells and have already proven useful in infectious diseases and cancer. In this case new, often chimeric, signaling receptors are expressed on $\mathrm{T}$ lymphocytes. The benefit of using $\mathrm{T}$-cells is their effector and regulatory functions, their ability to grow well in vitro and traffic to most sites of the body. Chimeric receptors containing extracellular domains from MHC class I linked to a signaling 
domain from TCR can be retrovirally transduced in T-cells or T-cell hybridoma. Upon encounter and recognition of their cognate TCRs, these chimeric receptors exhibit effector functions such as cytokine secretion, proliferation, or cytolysis depending on the type of $\mathrm{T}$ cell in which they are transduced $\left(\mathrm{CD} 4^{+}\right.$or $\left.\mathrm{CD} 8^{+}\right)(58)$.

A more specific surrogate receptor able to target autoreactive, encephalitogenic Th1 cells has recently been designed. This chimeric receptor contains the extracellular and transmembrane domains of mouse MHC class II I-A $\beta$ and $\alpha$, the zeta cytoplasmic signaling domain, and also an antigenic peptide, MBP89-101, linked on its surface. This receptor not only recognizes the cognate TCR but can also be stimulated as a result of this interaction. Among outcomes of this TCR-TCR interaction, CD8 ${ }^{+}$T-cells transduced with retrovirus containing this construct can specifically kill CD $4^{+} \mathrm{Th} 1$ antigen-specific T-cells. Although the chimeric receptor was designed to only carry one peptide epitope, experimental results show that this approach could also address the main problem of EAE, epitope spread, even when RMTC are administered one month after disease induction (59). One benefit of this approach is that it does not interfere with the whole immune system, but selectively targets encephalitogenic, antigen-specific cells. 


\section{Chapter 2. Development of chimeric receptors}

Chimeric receptors (CRs) are hybrid combinations of a recognition domain (variable regions of an Ig or a MHC molecule) and a signaling domain (TCR moieties responsible for signal transduction). The name "chimeric" resides in their mixed structure: CRs carry a recognition domain containing variable regions in charge of antigen recognition and an intracytoplasmic domain responsible for signal transduction (60) (Figure 2-1).

For a better understading of how CRs were engineered, I will briefly discuss the structure of IgG and TCR.

IgG is composed of two identical heavy and two light chains (kappa or lambda). The light chain has a variable region and a constant one and the heavy chain has additionally two or three constant domains. The chains are kept together by disulfide bonds. When treated with papain, the Ig breaks into two equal fragments of 45-50 KDa called fragments of antigen binding (Fab) and a third fragment of $50 \mathrm{KDa}$ called crystalizable fraction $(\mathrm{Fc})$. The antigen binding site consists of the variable domains of the light and heavy chains.

TCR is also composed of two chains, alpha and beta, each of which has a variable and a constant region. Its role is to recognize antigen-MHC complexes. The signals triggered by antigen recognition are not transduced by the TCR but by two proteins, CD3 and zeta $(\zeta)$, that are noncovalently linked to TCR, forming the TCR complex. Upon recognition of peptide, a cascade of signals including tyrosine phosphorylation and activation of nuclear factor-kappa (NF- $\kappa \mathrm{B})$, nuclear factor of activated T-cells (NFAT), 


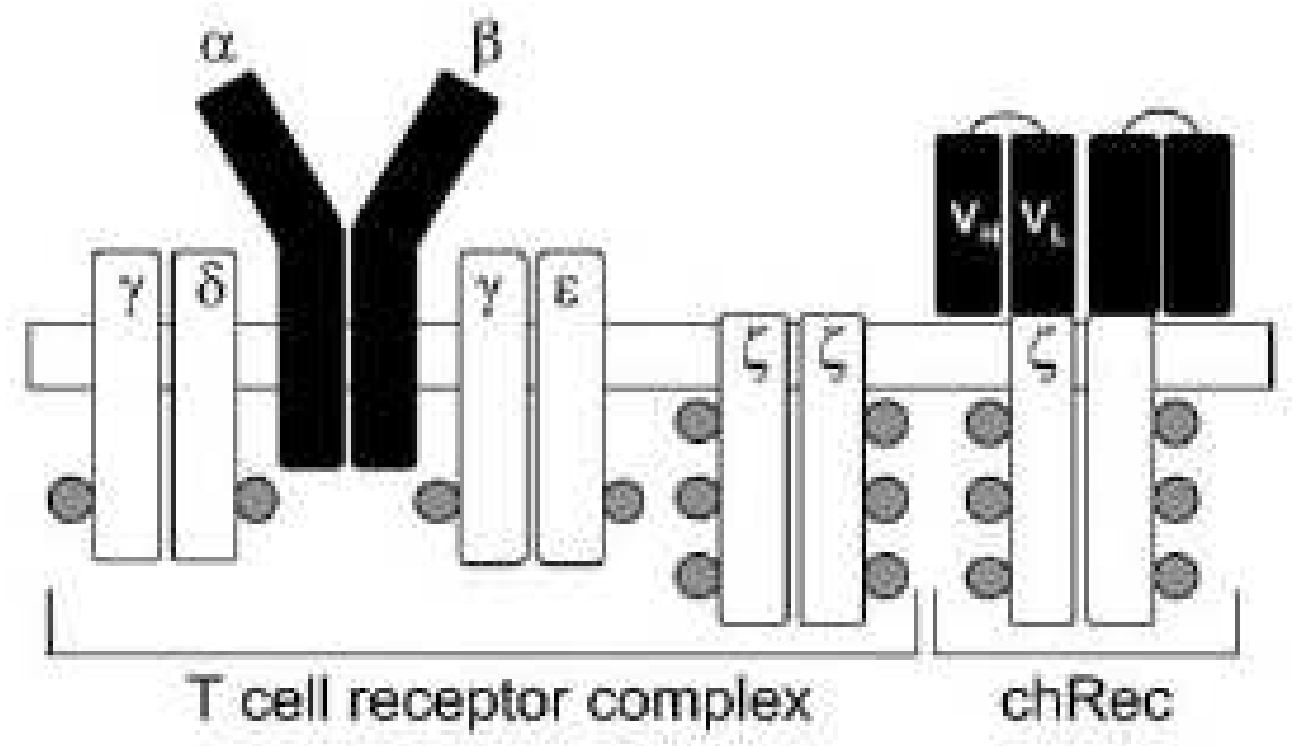

Figure 2-1. Structure of the T-cell receptor and a chimeric receptor. Reprinted with permission - Claudia Rössig, Malcolm K. Brenner. Chimeric T-Cell Receptors for the Targeting of Cancer Cells. Acta Haematol 2003;110:154-159. 
and activating protein-1 (AP-1), is triggered, eventually leading to cell proliferation and differentiation. The cytoplasmic domain of CD3 and $\zeta$ contain a conserved sequence called immunoreceptor tyrosine-based activation motif (ITAM) that plays a crucial role in signaling. Upon phosphorylation, ITAMs become docking sites for a tyrosine kinase called $\zeta$-associated protein of $79 \mathrm{kDa}$ (ZAP-70), ultimately leading to changes in gene expression in the T-cells.

CRs represent a smart combination between the ability of TCR and B cell receptor $(\mathrm{BCR})$ to recognize different antigens and elicit intracytoplasmic signal transduction events leading to different effects. Some of the earlier CRs were constructed by using both variable region of the heavy chain $\left(\mathrm{V}_{\mathrm{H}}\right)$ and variable region of the light chain $\left(\mathrm{V}_{\mathrm{L}}\right)$ combined with the $\alpha$ or $\beta$ constant domain of the TCR (61). Eshhar engineered a chimeric TCR composed of the variable region domain Fv of an antibody and the constant region of TCR. This receptor can be expressed as a transgene in T-cells via retrovirus, but due to the fact that two genes $\left(\mathrm{V}_{\mathrm{H}}\right.$ and $\left.\mathrm{V}_{\mathrm{L}}\right)$ have to be transfected into the same cell by two separate retroviral vectors, the efficiency of transduction was low. Consequently, this problem was overcome by joining together $\mathrm{V}_{\mathrm{H}}$ and $\mathrm{V}_{\mathrm{L}}$ into a single chain variable region $\left(\mathrm{scF}_{\mathrm{V}}\right)$ connected by a linker peptide $(62,63)$. The $\mathrm{scFv}$ is part of the extramembrane portion of the construct and is responsible for antigen recognition. This is linked to a region consisting of the gamma $(\gamma)$ or zeta cytoplasmic tail of the TCR. The two domains are linked by a hinge region that works as a spacer, increasing the distance between $\mathrm{scF}_{\mathrm{V}}$ and the plasma membrane. The hinge region belongs to IgG and accounts for the differences in the amino acid composition of the four classes of IgGs. It is placed between the $\mathrm{Fab}$ fragment and the constant $\mathrm{CH} 2$ and $\mathrm{CH} 3$ domains of the heavy 
chains and determines the flexibility of the $\mathrm{IgG}$ molecule. Flexibility is important for further effector functions of the IgG such as $\mathrm{C} 1 \mathrm{q}$ binding and complement activation (64); this feature and also the number of inter-heavy chain disulfide bonds in the hinge region is strictly characteristic and different for each $\mathrm{IgG}$ subclass. Different hinges were used, such as the hinge region of human $\mathrm{IgG} 1$ or $\mathrm{CD} 8$ or part of the extracellular region of CD28 some of which showing better expression in T-cells than others (65). The advantage of using the variable domain from Ig resides in the non-MHC restricted, antibody-type specificity that leads to a more ubiquitous array of specificities that can be transferred to T-cells through CRs.

Similar to the classic TCR, the mere contact between T-cells bearing chimeric receptors and target cells does not lead to cytolysis of latter. The CR can only guarantee specific recognition of target, but does not confer effector function to the T-cells unless they are activated upon this recognition. This may require the presence of a costimulatory signal and although a definite role has been established for induction of effector T-cells from naïve T-cells, it is not clear whether the costimulation is also required for the induction of effector cells from memory T-cells. The two signals theory states that T-cell activation requires recognition of antigen-MHC complex and costimulation; T-cells stimulated in the absence of costimulation are rendered tolerant rather than memory T-cells (66).

$\mathrm{CD} 28$ is a membrane protein with well-defined ability to promote T-cell proliferation and differentiation and induction of cytokine secretion upon binding of B7 molecules on APCs. The addition of the cytoplasmic domain of the co-stimulatory CD28 molecule to the engineered zeta signaling tail improved the efficacy of CR-transduced T- 
cells (67). Another co-stimulatory pathway is mediated by CD137 that belongs to the TNF family. Stimulation of CD 137 inhibits activation-induced cell death (AICD) (68).

CRs engineered as described can be transferred into human or murine T-cells and redirected against microbial antigens or tumor antigens in a manner independent of MHC restriction. Replication-defective viral vectors are used for transduction of CRs into Tcells. Several different vectors have been tried for this strategy, each with advantages and disadvantages. Adeno-associated vectors can be easily delivered into dividing and nondividing cells and have high transduction efficiency. Unfortunately, they integrate at low frequency and gene expression is, therefore, temporally limited (69). Retroviral vectors are better at integrating into the target genome but the target cell must be activated to incorporate the retrovirus. Future concerns have been raised as to whether viral integration may be oncogenic.

Pre-clinical trials using CRs for the therapy of infectious diseases have yielded controversial results. Human immunodeficiency virus (HIV) constitutes an example. Infusion of $\mathrm{CD}^{+}$and $\mathrm{CD} 8^{+} \mathrm{T}$ lymphocytes transduced with a chimeric receptor containing the extracellular domain of human CD4 linked to the zeta chain of TCRs were followed by a decrease in the viral load and an increase in $\mathrm{CD} 4^{+} \mathrm{T}$-cell counts. Upon recognition of TCR on the surface of HIV-infected T-cells, retrovirally-transduced Tcells get activated and exhibit effector function such as cytokine production, antigenspecific proliferation, and cytolysis of target cells (70). Other studies contradict these results and did not show any change in the HIV p24 or RNA plasma levels in patients that received cytotoxic T lymphocytes (CTL) transduced with CD4- $\zeta$ chimeric receptor, which indicates a lack of correlation between their in vitro and in vivo cytolytic 
capacity (71).

Human cancer cells possess tumor associated antigens that can be recognized and bound by monoclonal antibodies. These antibodies can recognize the extracellular domain of these genes; an example is ERBB2 oncogene present in breast ovarian, gastric, and colon cancers. $\mathrm{CR}$ bearing scFv derived from the ERBB2 antibody linked to a hinge region and the zeta cytoplasmic domain were engineered, followed by transduction into CTL. The newly modified CTL were capable of efficient in vitro lysis of fibroblasts and epithelial cells transfected with human ERBB2 oncogene. Adoptive transfer of both target cells and CTL into nude mice slowed ERBB2 tumor growth for ten days (72).

Based on the ability of monoclonal antibodies to recognize tumor associated antigens (TAA), cytotoxic T lymphocytes can be redirected using these antibodies in a clinical trial for metastatic ovarian cancer. One-third of the patients showed objective clinical responses, but the approach failed in most of them due to the limited accessibility of the solid tumors by antibodies, dissociation of antibodies from CTL, and the limited ability of re-directed T-cells to kill more than one cell (73). Another clinical trial using chimeric receptors was directed against renal cell cancer, an immunogenic tumor, with a specific monoclonal antibody, G250, that recognizes a carboxy-anhydrase expressed on the cell membrane in both primary tumors and metastases. A CR was engineered, bearing the scFv domains of G250 linked to the $\zeta$-chain from Fc receptor of IgE and was administered to G250 positive patients whose metastatic lesions were not amenable for resection in a phase I clinical trial protocol. Although infusions of T-cells retrovirally transduced with CR were clinically well tolerated, the patients developed liver toxicity and hyperbilirubinemia, but these laboratory abnormalities were reversible. This 
phenomenon occurred due to the interaction of G250 antibody on the surface of genemodified T-cells with G250L target antigen also expressed on the cells lining the bile ducts. The CR-transduced T-cells were detected in the peripheral blood and showed increased specific cytolysis against G250L target cells and increased secretion of IFN- $\gamma$ upon chimeric receptor stimulation (74). Another report has shown that a chimeric receptor designed to specifically recognize and target EBV was detected even 18 months after injection of EBV-specific CTL into patients (75). The long-term persistence and effects of therapeutic T-cells brings further hope for therapy with CRs.

Another caveat of immunotherapy with chimeric receptors is their functional limitations. There's certainly an advantage of CRs designed to carry the variable region of an antibody as recognition domain and the zeta cytoplasmic moiety for signal transduction in the fact that they can activate T-cells to trigger antigens regardless of their MHC restriction. Still, there are differences between the interaction of CR-bearing Tcells with their targets and a "classic" TCR interaction with a peptide held on a MHC molecule. These differences can lead to important functional issues. For the most part, upon recognition of a MHC-peptide complex, the CD4 or CD8 coreceptors are also recruited and interact with the nonpolymorphic regions of MHC class I or II, thus bringing lck (a tyrosine kinase from the Src family located in their cytoplasmic domain) in close association with ITAMs on the $\mathrm{CD} 3$ or $\zeta$ chains, leading to augmented activation of transcription factors and ultimately a more potent T-cell response. We can therefore assume that incorporation of CD4 or CD8 coreceptors might enhance the strength of CRs. Therefore, new CRs have been designed comprising multiple tandem linked signaling domains. These include zeta, CD4, CD28 with or without lck in different combinations 
(zeta only, CD4-zeta, CD28-zeta). The presence of lck promotes signaling by CRs because it enhances receptor phosphorylation. CD4 cytoplasmic tail does not possess intrinsic kinase activity, but in the form of CR-CD4-zeta, it was shown to be able to enhance the phosphorylation of CR by recruitment of p56lck to the CR similar to the way lck on CD4 coreceptor is recruited by TCR. Overall, the novelty of this particular model of CRs is the improved signaling ability upon recognition of antigen when either CD4 or CD28 signaling regions are incorporated. T-cells transduced with these type of CRs show better proliferation and cytokine production than the ones having $\zeta$ only as signaling domain. Among all the combinations tried, the chimeric receptor with a CD28- $\zeta$-lck intracellular signaling domain revealed to be the most efficient regarding IL-2 production and sensitivity to stimulation. However, its low surface expression level limited the practicality of using this receptor (76).

An issue to be considered in the evaluation of therapeutic T-cells bearing CRs is the difference between their excellent in vitro effects and the poor in vivo survival and expansion of CR-expressing T-cells. For in vivo survival of transduced T-cells, proper stimulation and expansion is vital since prolonged culture of these cells might diminish their functional effects. Thus, the essence is fulfillment of the right conditions for T-cell stimulation since insufficient amounts of cytokines lead to passive cell death, whereas inappropriate stimulation leads to AICD. Another problem might be the need for $\mathrm{CD} 4^{+} \mathrm{T}$ cells presence. $\mathrm{CD}^{+} \mathrm{T}$-cells can exercise their role in antigen clearance in the absence of any help in short-term acute infections. Chronic infections last longer and take more time to clear and $\mathrm{CD}^{+}$lymphocytes are required to sustain virus-specific $\mathrm{CD} 8^{+} \mathrm{CTL}$ (77). For example, $\mathrm{CD}^{+}$lysis ability seen in the late stages of acquired immunodeficiency 
syndrome (AIDS) is lost in correspondence to a dramatic drop in the number of $\mathrm{CD}^{+} \mathrm{T}$ cells.

In conclusion, adoptive immunotherapy with receptor-modified T-cells bearing chimeric receptors on their surface comprises a potential novel and specific therapy for malignancies and infectious diseases. 


\section{Chapter 3. Significance of a dileucine motif in CD28-zeta $(\zeta)$-containing chimeric receptors}

\subsection{Introduction}

Lysosomes are the ultimate destination of macromolecules transported from the extracellular space or cell membrane by endocytosis. These organelles can be accessed via internalization of carrier proteins into endosomes and then transportation to the lysosomes or via the biosynthetic pathway that involves an intermediate organelle, the trans-Golgi network, followed by intracellular delivery to endosomes and then lysosomes. Sorting of transmembrane proteins to endosomes and lysosomes is mediated by signals present in the cytosolic domain of the proteins. These signals include short amino acid sequences that can be tyrosine-based or dileucine motifs. There are two consensus dileucine motifs, [DE]XXXL[LI] or DXXLL. Dileucine (LL) motifs only have four to seven amino acid residues, but only two or three of them are critical for their function. These are recognized by proteins that play an important role in the endosomallysosomal system. Clathrin coats forming around plasma membrane contain heterotetrameric adaptor protein (AP) complex AP-2 and other accessory factors. Endosomal clathrin coats and the trans-Golgi network contain AP-1 and ADPribosylation factor-binding proteins (GGA1, 2, and 3) and monomeric adaptors. [DE]XXXL[LI] sorting signals are recognized by the $\mu$ and $\beta$ subunits of AP-1, AP-2, AP-3, and AP-4, leading to internalization, lysosomal, and basolateral targeting. DXXLL are recognized by the VHS domain GGAs, leading to sorting from the trans-Golgi to endosomes (78). 
The dileucine motifs have been identified in multiple proteins in a quest to characterize protein motifs responsible for lysosomal targeting. For example, to eliminate multiple targeting signals, Letourneur engineered chimeras containing the extracellular and transmembrane domain of IL-2 receptor antigen Tac (the alpha chain of the IL-2 receptor) linked to the cytoplasmic domain of each CD3 chain. Using these chimeras, a new dileucine-based targeting sequence in the cytoplasmic domain of CD3 $\gamma$ and $\delta$ was revealed, responsible for both rapid internalization and delivery to lysosomes (79). This sequence shown to be important in lysosomal targeting contains six amino acids, DKQTLL; site-directed mutagenesis of either of the leucines L130 or 131 established their ranking. The first leucine is invariant since replacement with any other amino acid lead to decreased signal, whereas the second leucine could be replaced by isoleucine without affecting the function.

\subsection{1 [DE]XXXL[LI] signals}

The $[\mathrm{DE}] \mathrm{XXXL}[\mathrm{LI}]$ signals are important in the sorting of many transmembrane proteins, such as: CD3- $\gamma$ (human Tm-8-SDKQTLLPN-26), tyrosinase (human Tm-8EEKQPLLME-12), CD4 (human tm-12-SQIKRLLSE-17), etc. In CD3- $\gamma$ chain, SDKQTLL sequence plays a part in serine phosphorylation-dependent downregulation of the TCR from the surface that involves rapid internalization and lysosomal degradation. CD4 has a very similar LL-containing sequence.

As previously mentioned, $[\mathrm{DE}] \mathrm{XXXL}[\mathrm{LI}]$ signals are recognized by the $\mu$ and $\beta$ subunits of various AP complexes. Each [DE]XXXL[LI] signal has a preference for a specific AP complex but not others. Although they bind to the $\mu 2$ subunit of AP (similar 
to tyrosine-based motifs), these determinants do not compete with each other.

\subsubsection{DXXLL signals}

DXXLL signals constitute a particular type of sorting determinants present in proteins or transmembrane receptors that cycle between the trans-Golgi and endosomes, such as cation-independent (CI-MPRs) or cation-dependent mannose-6-phosphate receptors (CD-MPRs). These signals mediate incorporation into clathrin-coated vesicles that move from the Golgi apparatus to the endosomal system. Similar to [DE]XXXL[LI] signal, this has a very strict requirement for the LL and also D residues, because mutations of any of these amino acids inactivate signaling and increase the protein expression at the cell surface (80). This signal does not bind to AP complexes but instead is recognized by the amino terminal VHS domain of GGAs, which are ADP-ribosylation factor-dependent clathrin adaptors within the trans Golgi and endosomes (81). This recognition is very specific since the VHS domain cannot bind the other dileucine motif, [DE]XXXL[LI], or the tyrosine motif YXXø. Regulation of the recognition of DXXLL signals also involves serine residues in a consensus sequence placed two or three amino acids upstream of DXXLL signals that are phosphorylated by casein kinase II (CK II). Upon phosphorylation of serine, the negatively charged oxygens in the phosphate group interact with the positively charged residues within the VHS domains of GGAs. Upon this interaction, MPRs are incorporated into clathrin-coated vesicles emerging from the trans-Golgi network. These vesicles will then deliver the receptors to endosomes and eventually, to lysosomes (82). Another interaction involves GGA 1 and 3 that also bear DXXLL motifs able to bind their own VHS domain (83), followed by auto-inhibition. 
Therefore, GAs should be dephosphorylated in order to displace from their own VHS domain, making it available for interaction with domains in the cytosolic part of other proteins.

\subsection{Identification of a murine CD28 dileucine motif that suppresses single-chain chimeric $\mathbf{T}$-cell receptor expression and function (1)}

\subsubsection{Introduction}

RMTC are T-cells modified to express surrogate chimeric receptors that can target antigens not normally recognized by the immune system. These chimeric receptors that redirect therapeutic RMTC against their targets substitute for the classic $\mathrm{T}$ cell receptor. They recognize target antigen through an extracellular antigen-recognition domain, such as a single-chain Fv fragment, and signal through a TCR-derived signal transduction domain, such as the TCR $\zeta$ chain (84). RMTC have shown therapeutic properties in cancer of infectious diseases systems, selectively targeting malignant or infected cells in model systems. No significant toxicity has been observed in phase I clinical trials (85).

A possible problem in redirecting therapeutic cells with chimeric receptors is the limited signal the receptors are able to transduce. The physiologic coreceptor and costimulatory signals that are normally delivered to T-cells when they interact with an APC are not necessarily available in the case of RMTC engaging with the ligand on a target cell. These signals are important, for they promote $\mathrm{T}$ cell survival, proliferation, and effector function. To avoid this drawback, we have developed single-chain chimeric 
receptors that incorporate moieties from both the TCR and costimulatory and/or coreceptor molecules. We decided to genetically link the signaling domain of the CD28 costimulatory molecule to the cytoplasmic tail of the TCR $\zeta$ chain. Several studies have shown that RMTC that express chimeric receptors composed of a CD28- $\zeta$ signaling region showed improved functional responses compared to those that only bear the $\zeta$ signaling tail.

In our study, we used RMTC to specifically target T lymphocytes. Some RMTC may be useful in transplantation or other settings. The specificity of the TCR is the defining feature of a pathologic T cell. In transplantation, these TCR are generally directed against allogeneic major histocompatibility complex (MHC) or syngeneic MHC linked to minor histocompatibility antigens. We designed surrogate CRs to specifically redirect RMTC against class I MHC-restricted T-cells. These CRs include the extracellular and transmembrane domains of the MHC class I K ${ }^{\mathrm{b}}$ molecule linked to either a murine $\zeta$ or $\mathrm{CD} 28-\zeta$ signaling tail. The $\mathrm{K}^{\mathrm{b}}$ extracellular region serves as bait for $\mathrm{K}^{\mathrm{b}}-$ restricted T-cells; the signaling domain promotes the RMTC's effector functions. Biochemical analysis of CR-mediated signal transduction in $\mathrm{K}^{\mathrm{b}}-\mathrm{CD} 28-\zeta$ or $\mathrm{K}^{\mathrm{b}}-\zeta-$ transduced T cell hybridoma showed that the presence of CD28 enhanced receptor phosphorylation and calcium flux. Furthermore, the CD28 domain allowed direct receptor association with the src kinase p56lck, critically involved in initiating and sustaining receptor-mediated signal transduction. $\mathrm{K}^{\mathrm{b}}-\mathrm{CD} 28-\zeta \mathrm{T}$ cell hybridoma also showed increased IL-2 production and signaling sensitivity.

Unlike these data with hybridoma, when we transduced primary murine $\mathrm{T}$ lymphocytes with the $\mathrm{CD} 28-\zeta$ or the $\zeta-\mathrm{CR}$, we did not observe significant differences in 
chimeric receptor-mediated functional responses (82). We further observed a 2-fold to 4fold decrease in the surface expression level of the $\mathrm{K}^{\mathrm{b}}-\mathrm{CD} 28-\zeta \mathrm{CR}$ in primary T-cells when compared with the $\mathrm{K}^{\mathrm{b}}-\zeta$ receptor. Therefore, addition of the CD28 costimulatory molecule in chimeric receptor leads to conflicting effects: it provides an enhanced signal into RMTC but at the same time diminishes the receptor surface expression and thus limits the extent and/or duration of this signal.

In order to find a sensible explanation for the decreased expression of the $\mathrm{K}^{\mathrm{b}}$ CD28- $\zeta$ CR, we analyzed the sequence of the murine CD28 cytoplasmic tail and we noticed a noncanonical dileucine internalization motif. Dileucine motifs have been well characterized in other proteins, but not CD28. To clarify the role of this motif in the CD28- $\zeta$ CR function, we inactivated it by mutating leucine to glycine, $[\mathrm{L} \rightarrow \mathrm{G}]$. We found that this mutation increased surface expression of the $\mathrm{K}^{\mathrm{b}}-\mathrm{CD} 28-\zeta$ receptor 2 -fold to 5 -fold compared with the wild type receptor. Moreover, $\mathrm{K}^{\mathrm{b}}-\mathrm{CD} 28[\mathrm{~L} \rightarrow \mathrm{G}]-\zeta$-modified T-cells showed increased sensitivity in cytokine production, proliferation, and cytolysis of target cells when compared with $\mathrm{K}^{\mathrm{b}}-\mathrm{CD} 28-\zeta \mathrm{RMTC}$. Therefore, this study identifies a previously undescribed dileucine motif within the murine CD28 tail and demonstrates its specific role in the restriction of CR function in RMTC.

\subsubsection{Materials and methods}

\section{$\underline{3.2 .2 .1 \text { Construct }}$}

cDNA clones or splenic cDNA were used to isolate cDNA fragments encoding the extracellular and transmembrane domain of the $\mathrm{H}-2 \mathrm{~K}^{\mathrm{b}}$ molecule and the cytoplasmic 
tails of murine CD28 and $\zeta$ by PCR. Flanking restriction sites were added to the fragments by PCR and the dileucine mutation was introduced by PCR mutagenesis. The constructs were assembled and subcloned into the MSCV retroviral vector that contains an internal ribosome entry site (IRES) linked to the green fluorescence protein (GFP) (gift from E. Vanin, St Jude Children's Research Hospital). Prior to ligation of all genes into the final construct, all DNA sequences were confirmed by sequencing at the St Jude Hartwell Center for Biotechnology.

\subsubsection{Antibodies, cells, mice}

TG-B transgenic mice were used as a source of $\mathrm{CD}^{+} \mathrm{T}$-cells for transducing the two constructs. These mice are transgenic for a rearranged SV40-T/H-2 $\mathrm{K}^{\mathrm{k}}$-restricted TCR that were bred more than 20 generations with B10.BR mice. As a source of target cells we used OT-1 mice (Jackson Laboratories, Bar Harbor, ME), transgenic for a rearranged ovalbumin 257-264/H-2K ${ }^{\mathrm{b}}$-restricted TCR. Antibodies included clone B20.1 anti-mouse $\mathrm{V}_{\alpha} 2$ (Pharmingen, San Diego, CA), clone 2C11 anti-mouse CD3e (gift from M. Blackman, Trudeau Institute, Saranac, NY), clone AF6-88.5 anti-mouse H-2K (Pharmingen and gift from M. Blackman), goat anti-mouse IgG (Jackson Laboratories), and goat anti-rat IgG (Jackson Laboratories).

\subsubsection{Retroviral transduction and T cell culture}

$10 \mu \mathrm{g}$ of CR constructs and $10 \mu \mathrm{g}$ of the retrovirus helper DNA PEQPAM (gift from J. Cleveland) were cotransfected into 293 T cells using calcium phosphate precipitation. After 16 hours, the cells were washed and cultured in Dulbecco modified 
Eagle medium/10\% fetal calf serum (DMEM/10\% FCS) for 48 hours. Supernatant was collected twice daily and used to infect GP+E86 retroviral producer cells in the presence of $8 \mu \mathrm{g} / \mathrm{mL}$ polybrene, for five days. Transduced GP+E86 cells were sorted for GPF by fluorescence-activated cell sorting (FACS). To transduce T lymphocytes, we isolated lymph nodes, processed them in a single-cell suspension, and stimulated them in vitro with soluble anti-CD3- and CD28 antibodies in the presence of $2 \mathrm{ng} / \mathrm{mL}$ recombinant murine IL-2 (rmIL-2) (R\&D Systems, Minneapolis, MN) for 48 hours. At that point, the medium was replaced with retroviral supernatant and $8 \mu \mathrm{g} / \mathrm{mL}$ polybrene, and the cells were spun at $1800 \mathrm{rpm}$ for 90 minutes in a Jouan CR422 tabletop centrifuge (Winchester, VA). Transduced T-cells were sorted for expression of GFP and CD8 and expanded in EHAA medium (Biosource International, Camarillo, CA) in the presence of rmIL-2 for up to 5 days. The cells were restimulated every 10 days using $2 \mu \mathrm{g} / \mathrm{mL}$ Concanavalin A (ConA; Sigma, St Louis, MO), 2 × 10\% $/ \mathrm{mL}$ irradiated syngeneic splenocytes $(3000 \mathrm{rad}$ ), and $2 \mathrm{ng} / \mathrm{mL}$ rmIL-2. The assays were performed on day five or six after stimulation, in the absence of exogenously added IL-2.

\subsubsection{Proliferation}

96-well plates were coated with goat anti-mouse IgG followed by loading of purified AF6-88.5 antibody. $5 \times 10^{4}$ CR-transduced T-cells and $2.5 \times 10^{5} 25$ irradiated syngeneic B10.BR splenocytes were added. After 48 hours, the cells were pulsed with 1 $\mu \mathrm{Ci}^{3}[\mathrm{H}]$-thymidine for 16 hours and harvested onto filtermats. Liquid scintillation counting of incorporated thymidine was used to assess proliferation. All samples were analyzed in triplicate and plotted as means. 


\subsubsection{Cytotoxicity assay}

RMTC were incubated overnight in medium with different concentrations of ovalbumin (OVA) 257-264 peptide (St Jude Hartwell Center for Biotechnology) in PBS or control PBS, then washed 3 times and resuspended in medium. Effector RMTC were incubated with $10^{5}$ OT-1 target T-cells in various ratios. The target cells were isolated from OT-1 TCR transgenic lymph node cells or splenocytes. After 5 to 6 hours of coincubation, 5000 to 10000 6- $\mu$ m fluorescent TruCount beads (Becton Dickinson, Franklin Lakes, NJ) were added. Samples were stained for $\mathrm{V}_{\alpha} 2$, washed once, and analyzed by flow cytometry. The target cells stained positive for $\mathrm{V}_{\alpha} 2$ and GFP negative, and were readily distinguished from the $\mathrm{GFP}^{+}$effector cells. The TruCount beads serve as a tool for normalization of cellular events and provide a quantitative assessment of the absolute number of target cells. Percent specific cytotoxicity was determined as $100 \times(1-$ viable target cell count after incubation with peptide-pulsed effectors/viable target cell count after incubation with unpulsed effectors). Parallel cultures of target cells in the absence of effector cells were performed simultaneously in all experiments as negative controls. Essentially identical results were obtained when cytotoxicity was alternatively calculated as $100 \times(1$ - viable target cell count after incubation with peptide-pulsed effectors/viable target cell count after incubation without effectors). All samples were analyzed in quintuplicate.

\subsubsection{Cytokine analysis}

IFN- $\gamma$ was analyzed using a Bioplex assay (Bio-Rad, Hercules, CA). 3000 analytical beads per well were added to a prewet 96-well filter plate. The samples were 
then added to the beads and incubated for 1 hour at room temperature (RT), followed by aspiration of supernatant, beads washing, and incubation for 1 hour with biotinylated antiIFN- $\gamma$ detection antibody. Detection was performed by staining with streptavidinphycoerythrin (PE) and fluorescence analysis with a Bioplex plate reader (Bio-Rad).

\subsubsection{Results}

\subsubsection{Design and expression of chimeric receptors}

$\mathrm{H}-2 \mathrm{~K}^{\mathrm{b}}$ extracellular and transmembrane domains were linked to the cytoplasmic domains of $\mathrm{CD} 28$ and $\zeta$ in order to engineer the wild-type $\mathrm{K}^{\mathrm{b}}-\mathrm{CD} 28-\zeta$ and dileucinemutated $\mathrm{K}^{\mathrm{b}}-\mathrm{CD} 28[\mathrm{~L} \rightarrow \mathrm{G}]-\zeta$ receptors (Figure 3-1). Both constructs were subcloned into the MSCV retroviral vector, which includes an IRES and GFP gene. Upon transduction of primary $\mathrm{CD}^{+} \mathrm{T}$ lymphocytes with retroviral supernatant, we observed efficiencies ranging from $15 \%$ to $50 \%$.

To determine the role of the dileucine motif in $\mathrm{CR}$ expression, $\mathrm{CD} 8^{+} \mathrm{GFP}^{+} \mathrm{T}$-cells transduced with either the $\mathrm{K}^{\mathrm{b}}-\mathrm{CD} 28-\zeta$ or $\mathrm{K}^{\mathrm{b}}-\mathrm{CD} 28[\mathrm{~L} \rightarrow \mathrm{G}]-\zeta$ receptor were sorted and stained with a $\mathrm{K}^{\mathrm{b}}$-specific antibody. We noticed a 2-fold to 5-fold increase in the surface expression level in the cells bearing the mutated receptor compared to the wild-type one, regardless the amount of GFP cotranscribed in the cells (Figure 3-2). These results demonstrate that the dileucine motif in the CD28 molecule significantly restricts the level of surface chimeric receptor, while its disruption enhances surface expression, but this finding does not necessarily ensure a more robust functional response. 


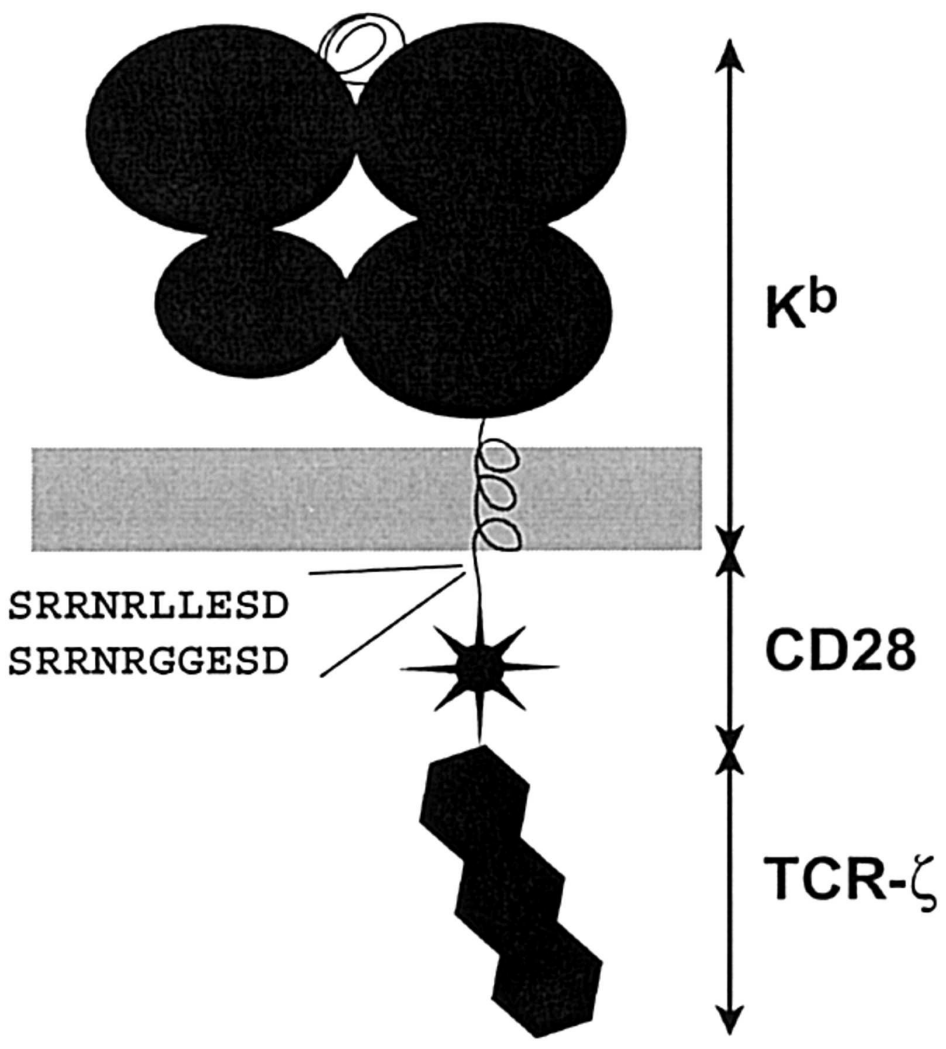

Figure 3-1. Chimeric receptor structure and sequence of the dileucine motif. Chimeric constructs were created by linking components in a cassette fashion. Extracellular and transmembrane domains are derived from the MHC class $\mathrm{I} \mathrm{H}-2 \mathrm{~K}^{\mathrm{b}}$ molecule. The murine CD28 and TCR- $\zeta$ cytoplasmic tails were attached as described. ${ }^{10}$ PCR mutagenesis was used to introduce the leucine to glycine change in the CD28 tail. This corresponds to an L184G and L185G conversion in the CD28 sequence (GenBank accession NP_031668). 


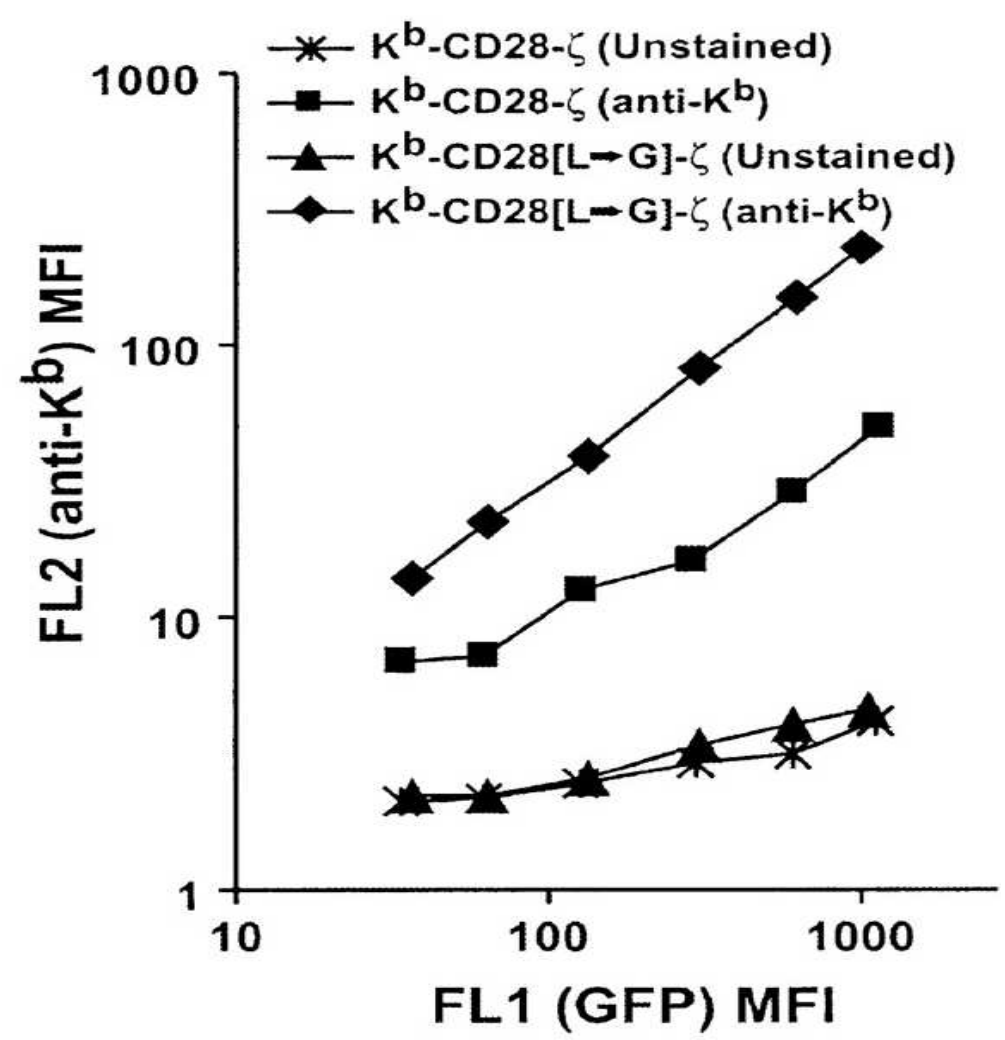

Figure 3-2. Increased surface expression of dileucine-mutated chimeric receptor. Chimeric receptor (anti- $\mathrm{K}^{\mathrm{b}}$ ) expression is plotted as a function of cotranscribed GFP level. Transduced cell populations were analyzed for GFP (FL1) expression level and gated into regions comprising approximately 0.2 to $0.3 \log$ fluorescence using Cellquest software (BD Biosciences, San Jose, CA). For cells within each of these gated regions, FL1 MFI (GFP) and FL2 MFI (anti- $\mathrm{K}^{\mathrm{b}}$ staining or control unstained) was calculated and plotted. 


\subsubsection{Functional response of RMTC}

Previous data has shown that the $\mathrm{T}$ cell response to stimulation depends on the intensity and duration of the stimulus. This implies that the increased expression of $[\mathrm{L} \rightarrow$ G] chimeric receptors should theoretically result in improved signaling compared with the wild type receptors. However, since the role of the dileucine motif in CD28 signaling has not been established yet, it is also possible that this disruption would hinder signal transduction. To determine whether this mutation proves beneficial on the functional response or not, we first measured $\mathrm{T}$ cell proliferation after stimulation through both receptors using a non-specific mitogen. $\mathrm{K}^{\mathrm{b}}-\mathrm{CD} 28-\zeta, \mathrm{K}^{\mathrm{b}}-\mathrm{CD} 28[\mathrm{~L} \rightarrow \mathrm{G}]-\zeta$, and MSCV retroviral control cells responded equivalently to Concanavalin A. This result demonstrates that the expression of CR does not affect the proliferative ability of therapeutic, CR-transduced T-cells in response to non-specific stimuli (Figure 3-3). In contrast, differences were observed after stimulation through the CR. T-cells transduced with the $[\mathrm{L} \rightarrow \mathrm{G}]$-mutated $\mathrm{CR}$ proliferated better than wild type CR-transduced T-cells in response to CR-specific stimulation. Therefore, we can conclude that the dileucine motif in CD28 functionally restricts chimeric receptor activity, and the $\mathrm{L} \rightarrow \mathrm{G}$ mutation alleviates this restriction.

To determine whether the enhanced function of mutated CR-transduced RMTC also affected the amount of cytokines produced by T-cells upon stimulation, we analyzed IFN- $\gamma$ secretion. A more than 3.5-fold IFN- $\gamma$ production was noted upon stimulation of $\mathrm{K}^{\mathrm{b}}-\mathrm{CD} 28[\mathrm{~L} \rightarrow \mathrm{G}]-\zeta \mathrm{RMTC}$ with CR-specific antibody (Figure 3-4). Therefore, disabling the dileucine motif also improves RMTC cytokine response.

It was already proven that $\mathrm{K}^{\mathrm{b}}-\mathrm{CD} 28-\zeta \mathrm{RMTC}$ can kill $\mathrm{K}^{\mathrm{b}}$-restricted target cells 


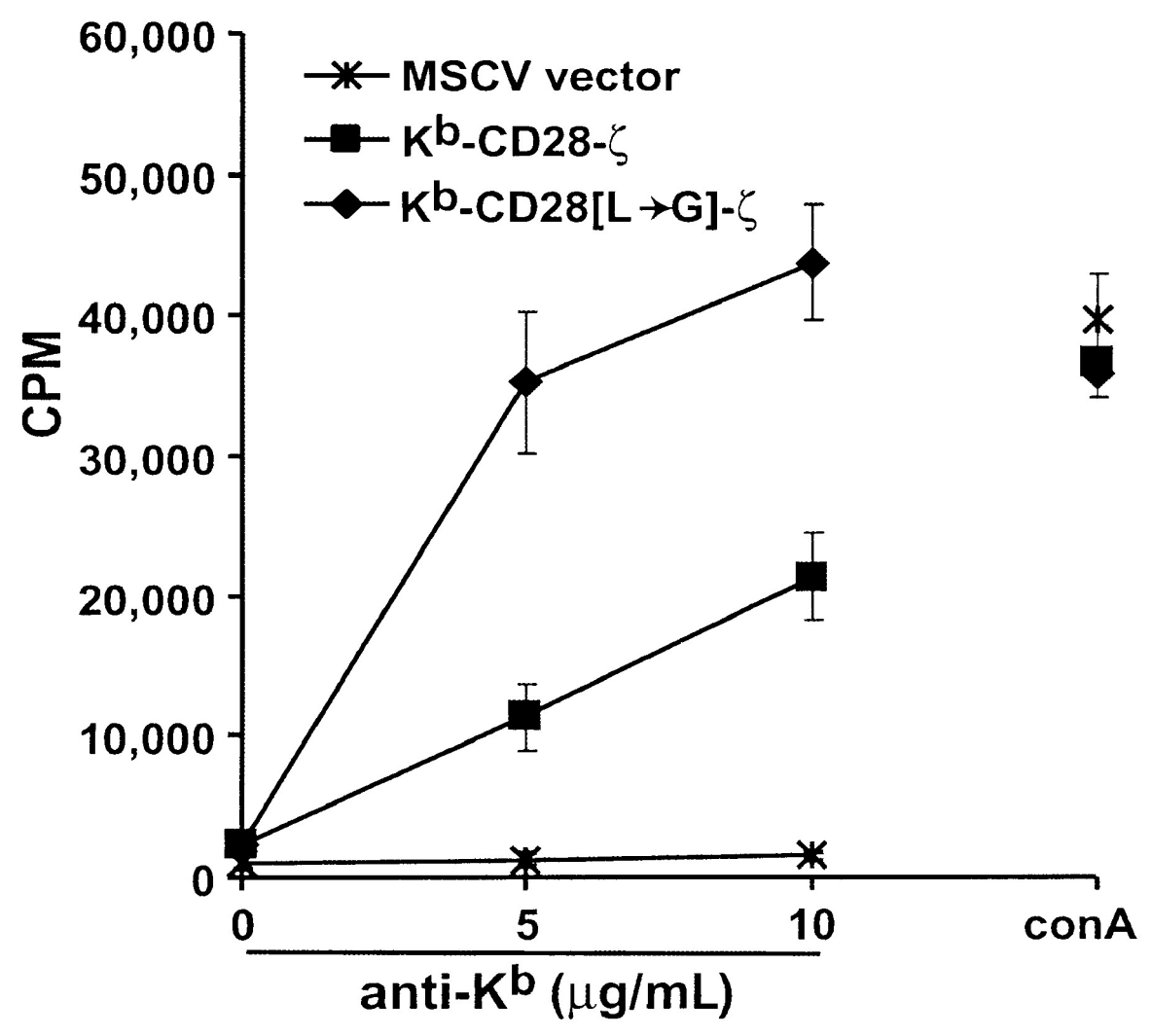

Figure 3-3. Proliferative response of $K^{b}-C D 28-\zeta$ and $K^{b}-C D 28[L \rightarrow G]-\measuredangle R M T C$. GFP-sorted $\mathrm{CD}^{+}$RMTC were stimulated 7 days after transduction with irradiated splenocyte feeders on plates coated with AF6-88.5 anti-H-2 $\mathrm{K}^{\mathrm{b}}$ or in the presence of the nonspecific mitogen conA. After 2 days the cultures were pulsed with ${ }^{3} \mathrm{H}$-thymidine and harvested 16 hours later. Data points are means of triplicate samples. Error bars show \pm 1 SD. One of 3 essentially identical experiments is shown. 


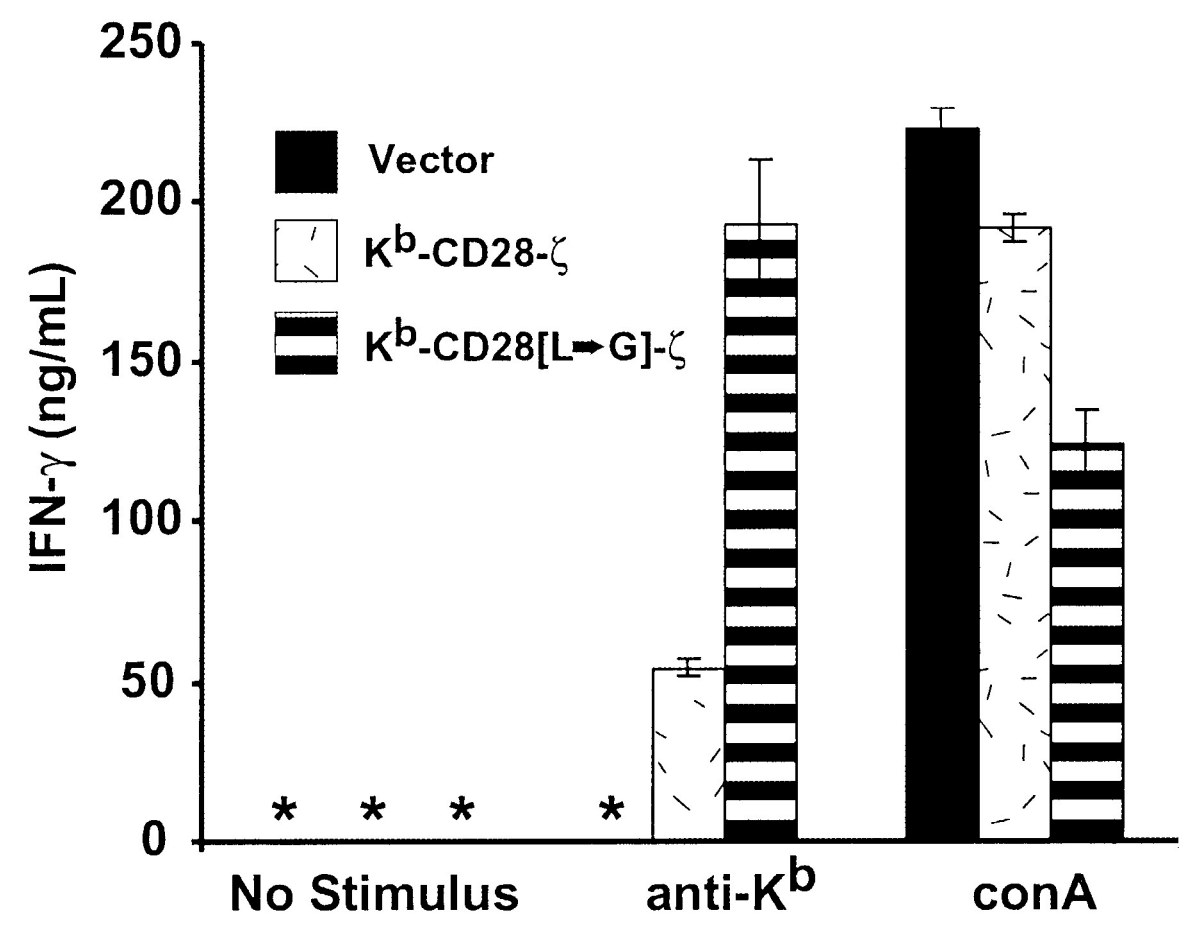

Figure 3-4. IFN- $\gamma$ production by $\mathrm{K}^{\mathrm{b}}-\mathrm{CD} 28-\zeta$ and $\mathrm{K}^{\mathrm{b}}-\mathrm{CD} 28[\mathrm{~L} \rightarrow \mathrm{G}]-\zeta$ RMTC. GFPsorted $\mathrm{CD}^{+}{ }^{+}$RMTC were stimulated 7 days after transduction in the presence of splenocyte feeders on plates coated with $5 \mu \mathrm{g} / \mathrm{mL}$ AF6-88.5 anti- $\mathrm{K}^{\mathrm{b}}$, with conA, or cultured in the absence of stimulation. Stimulation-induced IFN- $\gamma$ production was measured by Bioplex assay using anti-IFN-7-coated beads. Data points are means of triplicate samples. Error bars show $\pm 1 \mathrm{SD} ;{ }^{*}$, less than $1 \mathrm{ng} / \mathrm{mL}$. 
(58). We tested if the new mutated CR is more efficient in this aspect. We used OT-1 cells from the lymph nodes or spleen of mice transgenic for a TCR specific for $\mathrm{K}^{\mathrm{b}} / \mathrm{Ova}$ 257-264 as target cells. Ova peptide-pulsed $\mathrm{K}^{\mathrm{b}}-\mathrm{CD} 28[\mathrm{~L} \rightarrow \mathrm{G}]-\zeta \mathrm{RMTC}$ are able to specifically kill target OT-1 T-cells more efficiently than the $\mathrm{K}^{\mathrm{b}}-\mathrm{CD} 28-\zeta$ RMTC (Figure 3-5). This is a confirmation that the dileucine to glycine mutation enhances the effector function of RMTC, namely the ability to proliferate, secrete cytokines, and lyse Ovaspecific target cells.

The data above show that mutations in the dileucine motif of CD28 enhance the effector function of RMTC in vitro. We next wanted to find out if therapeutic cells were also effective in vivo. To do this, we coinjected Ova peptide-pulsed RMTC transduced with the wild type or mutated CR along with OT-1 transgenic cells labeled with carboxyfluorescein succinnimidyl ester (CFSE) into severe combined immunodeficiency (SCID) mice. Both $\mathrm{K}^{\mathrm{b}}-\mathrm{CD} 28[\mathrm{~L} \rightarrow \mathrm{G}]-\zeta$ and $\mathrm{K}^{\mathrm{b}}-\mathrm{CD} 28-\zeta$ peptide-pulsed RMTC efficiently eliminated OT-1 T-cells. This means that the mutated CR-transduced therapeutic cells are functional in vivo. These cells also show a slight - though not statistically significant increase in the cytolytic activity compared to the unmutated CR-transduced RMTC, which might be an indication for their preferential use in the future (Figure 3-6).

\subsubsection{Conclusions}

T-cells redirected against pathologic lymphocytes are potential tools in cellular immunotherapy. Therapeutic cells can migrate to different sites in the body, are longlasting, and exhibit different effector functions. Chimeric receptors composed of antigenrecognition and signaling domains from the TCR, such as the cytoplasmic $\zeta$ tail, were 

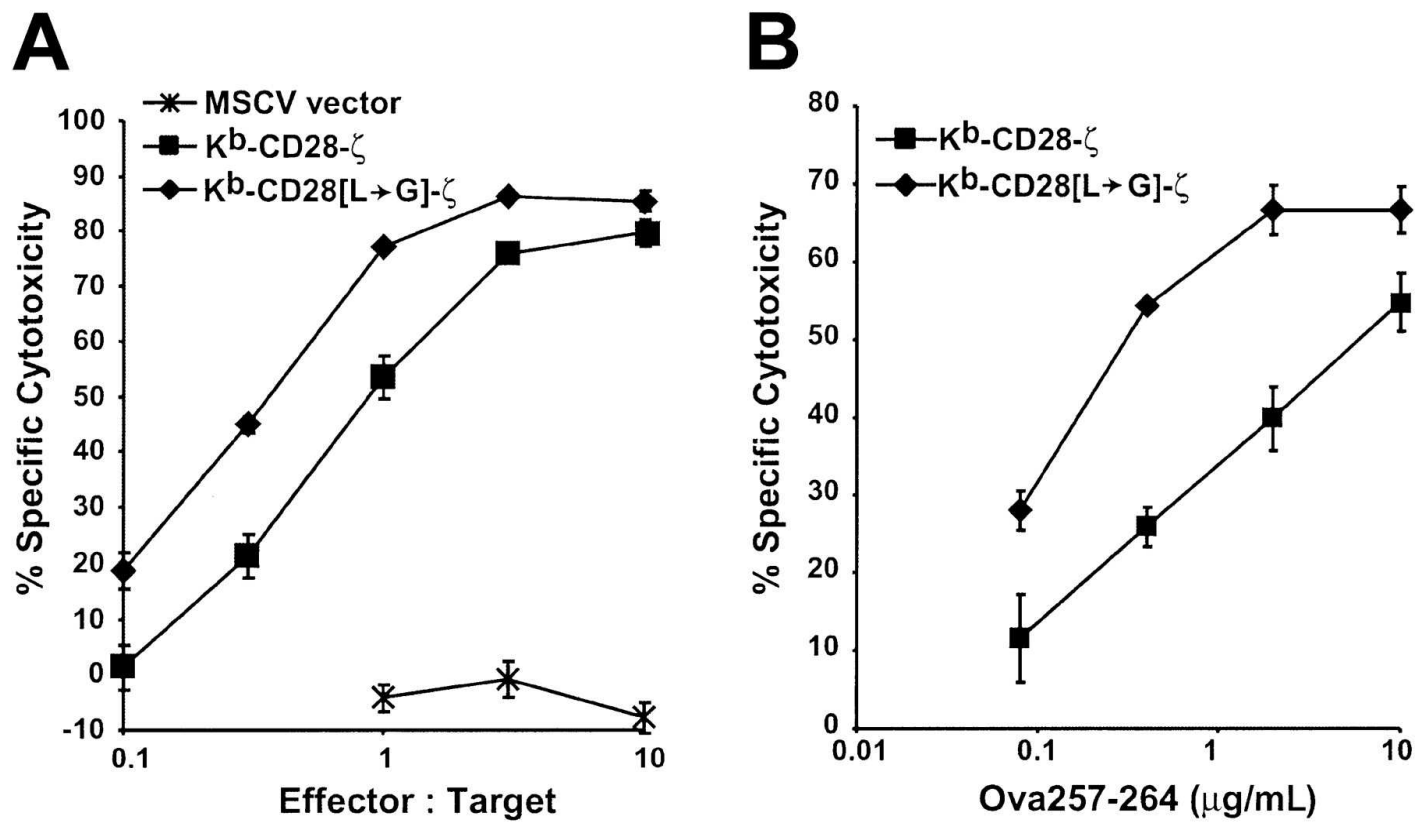

Figure 3-5. Cytolysis of antigen-specific T-cells by $\mathbf{K}^{\mathbf{b}}-\mathrm{CD28}-\zeta$ and $\mathrm{K}^{\mathrm{b}}-\mathrm{CD} 28[\mathrm{~L} \rightarrow \mathrm{G}]-\zeta$ RMTC. (A) RMTC were pulsed with $50 \mu \mathrm{g} / \mathrm{mL}$ ovalbumin 257-264 peptide or saline diluent, washed, and cultured for 6 hours with OT-1 TCR transgenic T lymphocytes at the designated effector-target ratio. Target cell survival was determined using quantitative flow cytometry. Specific cytolysis was calculated from the number of residual viable target cells in wells containing target cells pulsed with peptide compared with that in otherwise identical control wells including unpulsed effectors. (B) Similar to panel A, except experimental RMTC were pulsed with the designated concentration of ovalbumin peptide. All samples were cultured at an effector-target ratio of 1 . Data points are means of quintuplicate samples. Error bars show \pm 1 SD. Plots are representative of 3 independent experiments. 

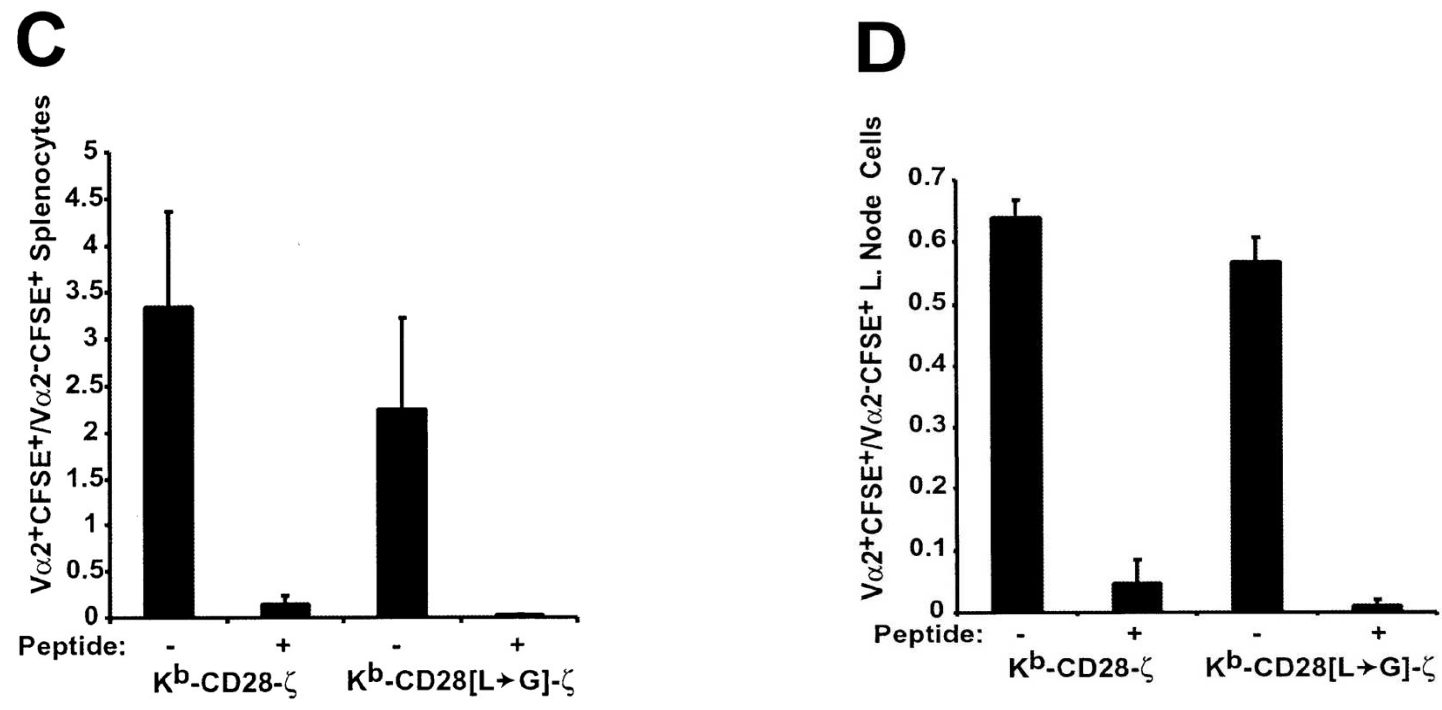

Figure 3-6. In vivo killing of antigen-specific $T$ lymphocytes using $K^{\mathrm{b}}-\mathrm{CD} 28-\zeta$ and $\mathbf{K}^{\mathbf{b}}$-CD28[L $\left.\_\mathbf{G}\right]-\zeta \mathbf{R M T C}$. A total of $10^{7}$ CFSE-labeled OT-1 lymph node cells were adoptively transferred intravenously into SCID mice; $10^{7} \mathrm{~Kb}-\mathrm{CD} 28-\zeta$ or $\mathrm{K}^{\mathrm{b}}-\mathrm{CD} 28[\mathrm{~L} \rightarrow$ G]- $\zeta$ peptide-pulsed or unpulsed RMTC were then adoptively transferred intravenously at an anatomically separate location. Twenty-four hours after transfer, spleen and mixed lymph nodes (mesenteric, cervical, axillary, inguinal) were isolated and single-cell suspensions prepared, stained with $\mathrm{V}_{\alpha} 2$-specific antibody, and analyzed by flow cytometry. (C) Normalized numbers of target cells in the spleens of treated animals. The ratio of residual transferred (CFSE-positive) RMTC targets ( $\mathrm{V}_{\alpha} 2$-positive) to nontargets $\left(\mathrm{V}_{\alpha} 2\right.$-negative) was calculated to control for the efficiency of adoptive transfer in mice treated with peptide-pulsed or control unpulsed effectors. (D) Analysis of LN cells. Error bars show \pm 1 SD. Results are representative of 3 independent experiments. 
proven effective in redirecting T-cells against target cells. Since their effector abilities depend upon the efficiency of signal transduction and the presence of costimulatory molecules, new generation of CRs include both the CD28 molecule and the $\zeta$ tail. Although we and others have observed enhanced function of the CR bearing the extracellular and transmembrane part of the $\mathrm{K}^{\mathrm{b}}$ molecule linked to $\mathrm{CD} 28-\zeta$ in $\mathrm{T}$ cell hybridoma, there was no noticeable difference between the functional effectiveness of this construct compared to the one lacking CD28 when transduced into primary murine T-cells. Also, the surface expression of this CR was diminished. We hypothesized that the poor expression resulted in the lack of improvement of their effector abilities. We here identify a novel dileucine motif in the murine CD28 molecule that hampers CR expression and function.

Up to now, two classes of dileucine motifs have been described, containing [DE]XXXL[LI] or DXXLL. They play critical roles in the sorting of transmembrane proteins. DXXLL signals are distinct dileucine-based sorting signals that cycle between the trans-Golgi network and endosomes and mediate incorporation into clathrin-coated vesicles that bud from the trans-Golgi network for transport to the endosomes. They bind to the GGA family of ARF-dependent clathrin adaptors localized in the trans-Golgi network. Mutations of either D or LL upstream inactivate the signals and result in increased surface expression of the proteins. [DE]XXXL[LI] motifs bind to AP-1, 2, or 3 and are less intolerant to mutations of the second isoleucine that can be mutated without impairing activity (74). The SRRNRLL that we discovered in the CD28 molecule is not identical to either [DE]XXXL[LI] or DXXLL motifs; rather, it is more similar to a [DE]XXXL[LI] motif that can also be found in other molecules, such as human CD4 
(SQIKRLLSE), mouse GLUT4 (RRTPSLLEQ) or human VAMP4 (SERRNLLED). This motif has a positively charged arginine residue upstream the dileucine motif instead of the canonical negatively charged amino acid residues.

Our experimental results disentangle the functional properties of the dileucine motif in CD28 molecule. Although disruption of this motif leads to enhanced surface expression and effector function, internalization might not be directly related to this fact. CD3 $\gamma$ molecule also has a SDKQTLL sequence responsible for internalization of the TCR but only after phosphorylation of serine (80). It is therefore, possible that internalization requires a conformational shift that makes the motif accessible to sortingassociated proteins. In our CR, the CD28 tail was taken out of its normal environment/structure, possibly making the dileucine motif more exposed to the proteinsorting apparatus, and leading to its constitutive activation.

The dileucine motif in CD28 limits the CR expression and signaling when transduced into primary murine T-cells. Mutation of leucine residues to glycine improves surface expression and upregulates proliferation, cytokine secretion, and cytolysis of target cells.

In addition to the in vitro evidence, we also provide evidence that effector T-cells bearing $\mathrm{CR}$ with mutated dileucine motifs are active in vivo, leading to selective killing of specific target cells. This constitutes a possible future therapeutic approach in transplant tolerance and autoimmunity. 


\section{Chapter 4. Development and function of humanized chimeric receptors}

\subsection{Introduction to the development of humanized chimeric receptors}

Surrogate chimeric receptors designed to bear an antigenic peptide linked to parts of MHC class I or II molecule and signaling parts of the TCR may be used as therapeutic tools (57). These CRs can be transduced into T lymphocytes giving rise to receptormodified T-cells designed to specifically target only pathogenic, peptide-specific T-cells. In initial studies our laboratory used a T-lineage specific promoter to express in transgenic mice a chimeric receptor that included the immunodominant epitope of myelin basic protein (MBP) in SJL mice linked to its restricting $\mathrm{I}-\mathrm{A}^{\mathrm{s}} \mathrm{MHC}$ and the signaling domain of TCR- $\zeta$. MBP-specific T-lymphocytes stimulated these transgenic RMTC, inducing effector functions, such as cytokine secretion, proliferation, and/or cytolysis of target cells. $\mathrm{CD}^{+}$, T helper 2 , or $\mathrm{CD} 4^{+} \mathrm{CD} 25^{+}$transgenic RMTC showed therapeutic activity in EAE, even after the dissemination of $\mathrm{T}$ cell responses through epitope spread (55). These data supported the application of RMTC immunotherapy in autoimmune diseases.

I have examined whether RMTC carrying humanized CRs can be similarly redirected against autoantigen-specific T lymphocytes. These humanized CRs were engineered by genetically linking autoantigenic peptide, MHC, and TCR- $\zeta$ or other signaling domains. The MHC-Ag serves as bait that specifically recognizes the TCR of autoantigen-specific T lymphocytes. Cognate TCR recognition of the extracellular MHCAg crosslinks the chimeric receptor, activating the RMTC through the receptor's signaling domains. 
Linkage analyses have associated HLA-DR2 (DRA*01/DRB1*1501) in patients of European descent with susceptibility to MS. Additionally, reactivity to a specific epitope, MBP84-102, restricted to this HLA has been well characterized in patients with MS (86). In this study we took advantage of this established association to design two chimeric receptors specific for DR2-restricted, MBP84-102-specific T-cells. Each heterodimeric receptor includes an extracellular and transmembrane domain comprised of HLA DRA*0101/DRB1*1501 with genetically linked MBP84-102 peptide. One receptor pair incorporates the cytoplasmic ITAM-rich domain of TCR- $\zeta$ on both the $\alpha$ and $\beta$ chains (MBP-DR2- $\zeta$ ). The second receptor pair lacks these signaling chains (MBPDR2), allowing us to define the role of receptor signaling in therapeutic cell function, which we were unable to do with the transgenic cells in our previous mouse model. The receptors were placed in an MSCV-based retroviral vector to allow us to determine whether adoptively transferred T-lymphocytes retrovirally transduced with the tricistronic $(\alpha, \beta$ receptor, GFP) chimeric constructs are functional, able to re-direct effector activity, and capable of modulating autoimmune disease in a humanized model system.

In this chapter, I describe our efforts to create humanized chimeric receptors and validate them through in vitro studies of the therapeutic cells' effector abilities. The receptors were transduced into two types of effector cells, immortalized $\mathrm{TCR}^{-\mathrm{CD}} 4^{-\mathrm{CD}} 8^{-}$ 4G4 T-cell hybridoma and primary $\mathrm{CD}^{+} \mathrm{T}$ lymphocytes. The activity of the receptors against cognate MBP84-102/DR2-specific T-cells was tested using either a hMBP84102-specific Ob1A12 T-cell hybridoma (or Ob hybridoma), a T-cell clone derived from a patient with relapsing-remitting multiple sclerosis, or T-cells from mice made transgenic with rearranged TCR derived from the Ob hybridoma, $\mathrm{Ob} \mathrm{Tg}$ mice (87). These $\mathrm{Ob} \mathrm{Tg}$ 
mice were bred with another strain that constitutively expresses human DR2 gene using the human DR2 promoter (Fug 5 mice) (88). As a negative control for our in vitro studies we used 6F11 T cell cell hybridoma specific for MBP89-101 epitope (same as hMBP 84102) restricted by $I-A^{s}$.

\subsection{Materials and methods}

\subsubsection{Design of humanized MBP-DR2-ל construct}

The MBP-DR2- $\zeta$ construct contains two polypeptides. The first one includes the extracellular and transmembrane domains of the human MHC class II beta chain DRB1*1501 linked to the human MBP peptide epitope 84-102 and the $\zeta$ cytoplasmic domain of the TCR. This was then linked to the human MHC class II alpha chain DRA*0101 paired with the $\zeta$ cytoplasmic tail of TCR. The alpha and beta chains were connected by a small amino acid sequence extracted from the Thosea asigna virus (TaV). We cloned the two genes in a single open reading frame by eliminating the stop codon at the end of the first $\zeta$ tail. The $2 \mathrm{~A}$ sequence allows for polycistronic message formation. As the mRNA is translated, the ribosome pauses at the $2 \mathrm{~A}$ sequence and cleaves the $\mathrm{N}$ terminal peptide from the nascent protein product. The ribosome does not release the mRNA however, and continues to synthesize the C-terminal polypeptide. Following cleavage, the $2 \mathrm{~A}$ peptide remains attached to the C-terminus of the first gene. The advantage of this bicistronic system using $2 \mathrm{~A}$ sequences is the same amount of two distinct proteins, $\alpha$ and $\beta$ chains of the chimeric construct, is produced (89). Internal ribosomal entry sites (IRES) can also be added to mRNA to permit for polycistronic 
message production, however, variable translational efficiencies are observed from the 5' mRNA cap and the IRES, leading to non-stoichiometric protein production. This synthetic heterodimeric gene was subcloned into the murine stem cells virus (MSCV) retroviral expression vector that contains an IRES-linked green fluorescent protein (GFP) for an easy identification of the gene by fluorescence activated cell sorting (FACS) analysis.

\subsubsection{Generation of leader sequence}

We started by extracting total RNA from MGAR cell lines using RNeasy procedure (Qiagen, Valencia, CA). MGAR cells are Epstein-Barr virus (EBV)transformed B cells that express DRA*0101/DRB1*1501. We used the following protocol:

$10 \times 10^{6}$ MGAR cells were counted and spun for 5 minutes in a centrifuge tube. The supernatant was discarded and $600 \mu$ lysis buffer RTL (Quiagen) containing 10 $\mu 1 / \mathrm{ml}$ 2-mercaptoethanol was added to the pellet which was then resuspended by vortexing. The disrupted cells were then homogenized by pipetting the lysate directly onto a QIAshredder column followed by a 2 minute-centrifugation in a microcentrifuge at maximum speed and collection of the homogenized lysate. One volume $(600 \mu \mathrm{l})$ of $70 \%$ ethanol was added to the homogenized lysate, mixed, applied to an RNeasy minispin column carried by a 2-ml collection tube, and centrifuged for 15 seconds at $8000 \mathrm{x} \mathrm{g}$. This step was repeated until the whole volume was used and the flow-through was discarded every time. $700 \mu \mathrm{l}$ buffer RW1 (Quiagen) was added to the column and centrifuged again like above, followed by transfer of the column onto a new collection 
tube and addition of $500 \mu \mathrm{l}$ buffer RPE (Quiagen) and another step of centrifugation. We repeated this step twice. The column was ultimately transferred again to a new collection tube and $30 \mu 1$ of RNase-free water was added to the RNeasy membrane to elute RNA. We obtained $194 \mu \mathrm{g} / \mathrm{ml}$ total RNA that was stored at $-80^{\circ} \mathrm{C}$.

After RNA extraction, we performed reverse transcription polymerase chain reaction (RT-PCR) using the standard Omniscript protocol (Qiagen) for first-strand complementary DNA (cDNA) synthesis as follows:

Tube 1: $10 \mu \mathrm{l}$ mix containing $2 \mu \mathrm{l}$ RNA (thawed on ice) and $8 \mu \mathrm{l}$ RNase-free water was heated at $65^{\circ} \mathrm{C}$ for 5 minutes.

Tube 2: $10 \mu \mathrm{l}$ mix of oligo $\mathrm{d}(\mathrm{T})$ primer (12 nucleotides) at a final concentration of $1 \mu \mathrm{M}, 10 \mathrm{x}$ RT buffer, dNTP mix, $1 \mu \mathrm{l}$ RNasine, RT enzyme, and water.

Tube 2 mix was added to tube $1 \mathrm{mix}$ and kept for an hour at $37^{\circ} \mathrm{C}$. The singlestranded cDNA obtained can be kept at $-20^{\circ} \mathrm{C}$ or PCR for the genes of interest using specific primers can ensue.

Exon 1 from HLA-DRB1 encodes the leader peptide (GenBank Accession \# NM 002124; accessed on April 3, 2003). A Kozak sequence for initiation of translation in vertebrates (90) was introduced by PCR and the whole piece was flanked by EcoRI and NheI restriction sites. The set of primers used for this reaction was:

Primer 1: 5'-GATCAGAATTCGCCACCATGGTGTGTCTGAAGCTCCCTGG EcoRI Kozak

Primer 2: 3'-TTCATCGCTAGCCAAAGCCAGTGGGGAGCTCAGCAC NheI

We set up a PCR using MGAR cDNA and this set of primers as follows: $100 \mu \mathrm{l}$ 
reaction mix containing $2 \mu \mathrm{l}$ cDNA, $10 \mu \mathrm{l}$ buffer and magnesium chloride $\left(\mathrm{MgCl}_{2}\right), 2 \mu \mathrm{l}$ dNTPs, $2 \mu \mathrm{l}$ primer $1,2 \mu \mathrm{l}$ primer 2 (at a concentration of $25 \mathrm{pmol} / \mu \mathrm{l}$ ), $0.5 \mu \mathrm{l}$ high fidelity Taq polymerase and $81.5 \mu \mathrm{l}$ water was submitted to 35 cycles of amplification using the following set-up:

First cycle of amplification: denaturation 5 minutes at $94^{\circ} \mathrm{C}$, annealing 2 minutes at $55^{\circ} \mathrm{C}$, extension 3 minutes at $72^{\circ} \mathrm{C} .34$ subsequent cycles followed using a gradient for the annealing step, starting at $65^{\circ} \mathrm{C}$ and subtracting $0.2^{\circ} \mathrm{C}$ each cycle until it reached $55^{\circ} \mathrm{C}$. We checked the size of the leader sequence on a $1 \%$ agarose gel and we obtained the expected size of 101 base pairs (bp). The PCR product was submitted for sequencing in a tube of $12 \mu \mathrm{l}$ total mix containing $1 \mu \mathrm{l}$ of DNA, $1 \mu \mathrm{l}$ of each primer, forward (primer 1) and reverse (primer 2) at $3.2 \mathrm{pmol}$, and water at the Hartwell Center, St. Jude Children's Research Hospital.

Once the product was proven correct, we used the two enzymes, EcoRI and NheI, to digest the leader sequence, gel purify it, and subclone it into pBluescript (pBS) II KS (+) vector (Stratagene, La Jolla, CA) digested with the same enzymes.

\subsubsection{Generation of MBP peptide sequence}

NCBI nucleotide search found the sequence for Homo sapiens MBP (GenBank Accession \# NM 002385; accessed on April 30, 2003).The MBP epitope 84-102 (DENPVVHFFKNIVTPRTPP) was synthesized as oligonucleotides by the Hartwell Center at St. Jude Children's Research Hospital, Memphis, TN. The oligonucleotides used have the following sequence:

Forward primer: 5'-CTAGCGATGAAAACCCCGTAGTCCACTTCTTCAAGA NheI 
Reverse primer: 3'-GATCCGCCTGACCCTCCGCCACCGGACCCGCCACCTCCG BamHI

GGTGGTGTGCGAGGCGTCACAATGTTCTTGAAGAAGTGGACTACGGGTTTTC ATCG

These two oligos were mixed in a 1:1 ratio, annealed at $94^{\circ} \mathrm{C}$, cooled down at room temperature, and ligated it together with EcoRI/NheI-digested leader sequence into pBS II KS vector using T4 DNA ligase (New England BioLabs, Ipswich, MA). This time, the vector was digested with EcoRI (that the leader sequence has a site for) and BamHI (present at the end of the peptide sequence or beginning of the beta chain). We ended up with the following piece of $185 \mathrm{bp}$ that we sequenced and confirmed to be correct:

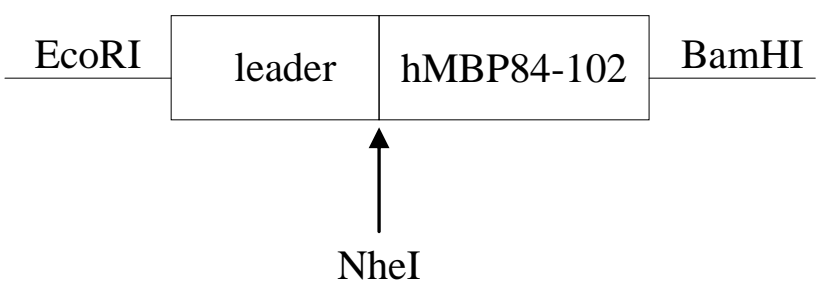

\subsubsection{Generation of HLA-DRB $1 * 1501$}

NCBI nucleotide search found the sequence for Homo sapiens HLA-DRB1 (GenBank Accession NM_002124 on May 5, 2003) mRNA with exons 2 and 3 coding for the extracellular domains. We amplified the cDNA obtained as previously described (4.2.1.1) with the following set of primers, adding two restriction sites, BamHI and AatII: Forward primer: 5'-TCAGGCGGATCCGGGGACACCCGACCACGTTTCTTG BamHI 
Reverse primer: 3'-TCTTGACGTCTGATTCCTGAAGTAGATGAACAGCCC

AatII

PCR amplification yielded a $682 \mathrm{bp}$ product. We subcloned this gene into pBS II vector digested with BamHI/AatII, sequenced this, and confirmed the correct sequence.

\subsubsection{Generation of the $\zeta$ chain}

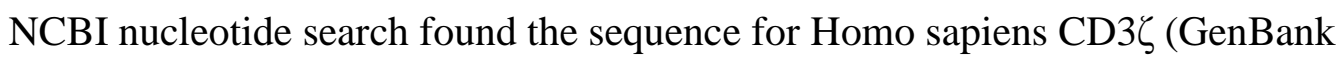
Accession NM_000734 on May 1, 2003)) mRNA. cDNA was provided by courtesy of Dr. Dario Campana, St. Jude Children's Research Hospital, Memphis, TN and amplified by PCR to obtain the cytoplasmic tail flanked by AatII and ApaI restriction sites using the following set of primers:

Forward primer: 5'-AATCAGACGTCAAGAGTGAAGTTCAGCAGGAGCGCA Aat II

Reverse primer: 3'-GCCATGGGCCCGGGATTTTCCTCCACGTCCCCGCATGTT Apa I

AGAAGACTTCCCCTGCCCTCGGCTCTGCGAGGGGGCAGGGCCTGCATGTGAA G

The reverse primer also included the reversed 2A sequence (discussed below), which was synthesized as part of the oligonucleotide. Similar to the other genes, the zeta2A gene was also sequenced, confirmed correct, and subcloned into pBS II vector. The product was $396 \mathrm{bp}$. 


\subsubsection{The $2 \mathrm{~A}$ sequence}

Thosea asigna virus $(\mathrm{TaV})$ is a member of the Tetraviridae family comprised of single-stranded RNA picornavirus whose polyproteins undergo co-translational cleavage to produce several proteins $(\mathrm{P} 1, \mathrm{P} 2, \mathrm{P} 3)$. The $2 \mathrm{~A}$ sequence is a proteinase that cleaves at its own amino terminus generating two protein products, $\mathrm{P} 1$ and $\mathrm{P} 2$. The $2 \mathrm{~A}$ sequence was previously used in retroviral vectors as an alternative system to IRES to generate a polycistronic message (91). In the MBP-DR2- $\zeta$ and MBP-DR2 constructs the 2A sequence is flanked by $\alpha$ and $\beta$ chimeric receptor chains forming a unique open reading frame. The cleavage of the polyprotein product occurs at the $\mathrm{C}$-terminus end of the $2 \mathrm{~A}$ sequence, leaving it fused to the upstream $\beta$ chain protein which is otherwise released intact. The amino acid sequence of the $2 \mathrm{~A}$ was:

\section{5'-RAEGRGSLLTCGDVEENPGP-3'}

We ligated the $\beta$ chain gene to zeta- $2 \mathrm{~A}$ and subcloned them into $\mathrm{pBS}$ II vector digested with BamHI and Apa I. We ended up with the following piece of $1078 \mathrm{bp}$ that was confirmed correct by sequencing:

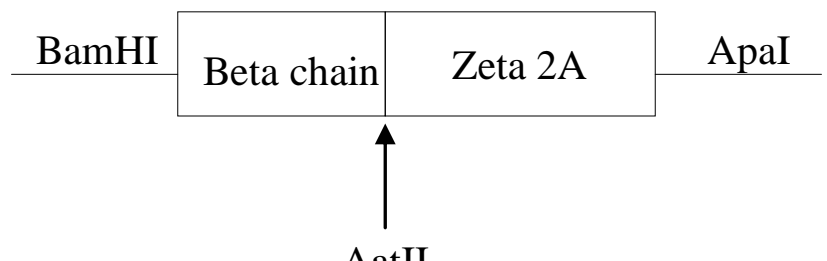

AatII

\subsubsection{Generation of HLA-DRA*0101}

NCBI nucleotide search found the sequence for Homo sapiens HLA-DRA (GenBank Accession NM_019111 on May 5, 2003) mRNA with exons 2 and 3 coding 
for the extracellular domains and exon 4 coding for the transmembrane domain and the cytoplasmic tail. cDNA produced from MGAR cells as described at 4.2.1.1 was used to isolated the DRA fragment. The extracellular and transmembrane domains of interest were flanked by ApaI and AatII restriction sites. The following set of primers was used for amplification of the two domains:

Forward primer: 5'-AATCCCGGGCCCATGGCCATAAGTGGAGTCCCTGTG ApaI

Reverse primer: 3'-CTCTTGACGTCAATCCCTTGATGATGAAGATGGTCCCAA AatII

PCR amplification yielded a 736 bp product. This gene was subcloned into pBS II vector digested with the two enzymes after sequencing and checking for the correct sequence.

\subsubsection{Generation of synthetic $\zeta$ tail}

Two zeta tails were required for the MBP-DR2- $\zeta$ construct, one linked to the $\alpha$ chain, the second linked to the $\beta$. For the $\beta$ chain, native $\zeta$ sequence was used. For the $\alpha$ chain, we chose to redesign the $\zeta$ tail by keeping the same amino acid sequence but changing the codons that determine each amino acid. This was to ensure that rearrangement between the two $\zeta$ chains would not occur during the production of retrovirus. Synthetic $\zeta$ was engineered as two sets of large oligos, one for the first half of the construct and the second one for the rest of the construct. The 3' end of the first set and the 5' end of the second set, respectively, had a PstI restriction site. The two sets are as follows: 
Set 1 - Forward primer: 5'-GGAGCGGATCCGACGTCCGCGTAAAATTTTCTCG \begin{tabular}{|l|l|} 
BamHI & AatII \\
\hline
\end{tabular}

TTCAGCTGATGCACCAGCTTATCAACAAGGACAAAATCAACTATACAATGAA CTGAACCTTGGTAGGCGTGAAGAATATGAC

Reverse primer: 3'-TTTGTCCTTCTGCAGCTCGTTATAAAGTCCCTCTTGTGGA PstI

TTTTTTGCGACGTGGTTTACCTCCCATTTCTGGATCTCTTCCTCTACGTTTAAT CTAG TACGTCATATTCTTCACGCCT

Set 2 - Forward primer: 5'-AACGAGCTGCAGAAGGACAAAATGGCAGAAGCA PstI

TATTCAGAAATCGGAATGAAGGGAGAAAGAAGACGAGGTAAAGGCCATGAC GGATTATAT

Reverse primer: 3'-GACTACTCGAGTTATTACCTTGGAGGTAATGCTTGCATA XhoI

TGTAAAGCATCATAAGTATCTTTTGTAGCCGTTGATAATCCTTGATATAACCG TCATGGCC

The two sets of primers were annealed and made double stranded using one PCR cycle ( 4 minutes denaturation at $94^{\circ} \mathrm{C}, 1$ minute annealing at $55^{\circ} \mathrm{C}, 3$ minutes extension at $72^{\circ} \mathrm{C}$ ) and high fidelity Taq polymerase, followed by purification and digestion with the two sets of enzymes, BamHI - PstI and PstI - XhoI for the second one. The two pieces of the synthetic $\zeta$ gene were then subcloned into pBS II vector digested with BamHI and XhoI. The product was 354 bp long.

This synthetic gene was then ligated together with the $\alpha$ chain and subcloned 
again into pBS II vector digested with ApaI and XhoI. We ended up with a gene of 1090 bp shown below that was confirmed correct by sequencing:

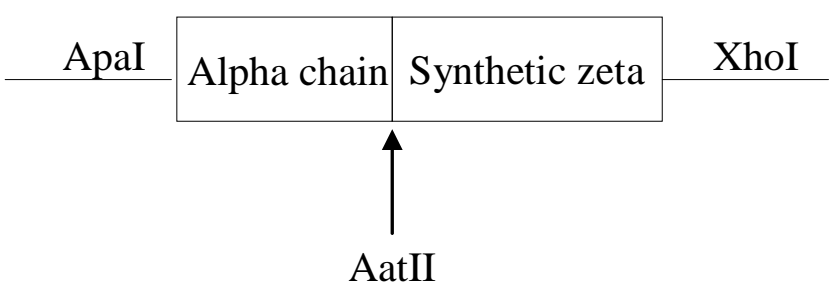

\subsubsection{Ligation into the final construct}

We have avoided the inconvenience of cloning six genes of different sizes into a considerably large vector such as $\operatorname{MSCV}(6.825 \mathrm{~Kb})$ by subcloning two genes at a time into a smaller vector, $\mathrm{pBS}$ II KS (+) of only $3 \mathrm{~Kb}$. We ended up with three pieces of the final product: leader-MBP, beta- $\zeta-2 \mathrm{~A}$, and alpha-synthetic $\zeta$ of 185, 1078, and $1090 \mathrm{bp}$, respectively. We then subcloned the leader-MBP and the beta-zeta-2A into pBS II. This step yielded the following clone of $1263 \mathrm{bp}$, shown below:

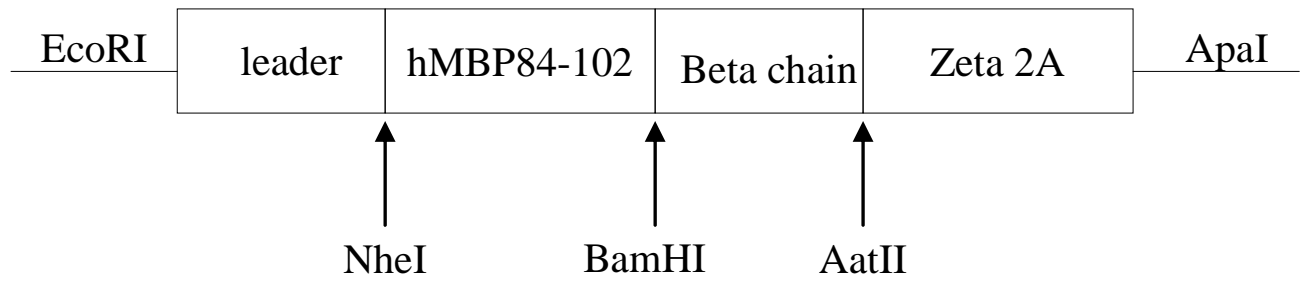

We digested this new gene with EcoRI/ApaI and inserted it along with the alphasynthetic $\zeta$ one digested with ApaI/XhoI into MSCV retroviral vector cut with EcoRI/XhoI that includes an IRES linked to GFP. The whole leader-MBP- $\beta-\zeta-2 \mathrm{~A}-\alpha-$ synthetic $\zeta$ construct was $2.352 \mathrm{~Kb}$. 


\subsubsection{Design of humanized tailless MBP-DR2 construct}

This signaling deficient construct was produced in order to clarify the role of CR signaling for RMTC function.

\subsubsection{Generation of leader-MBP peptide}

We used the same leader-MBP peptide gene that we previously used for the tailbearing construct restricted by the same sites, EcoRI and BamHI (see 4.2.1.1 and 4.2.1.2).

\subsubsection{Generation of HLA-DRB $1 * 1501$}

We amplified the cDNA with the following set of primers, adding the same BamHI as for the previous construct, but changing the second site to an ApaI. The reverse primer was a large oligonucleotide that also contained the $2 \mathrm{~A}$ sequence in a manner analogous to how we added $2 \mathrm{~A}$ to the $\zeta$ chain in the MBP-DR2- $\zeta$ construct:

Forward primer: 5'-TCAGGCGGATCCGGGGACACCCGACCACGTTTCTTG BamHI

Reverse primer: 3'-GCCATGGGCCCGGGATTTTCCTCCACGTCCCCGCATGTT Apa I

AGAAGACTTCCCCTGCCCTCGGCTCTGCGAGGGGGCAGGGCCTGCATGTGAA G

Similar to the other genes, the beta 2A gene was also sequenced and subcloned into $\mathrm{pBS}$ II vector. 


\section{$\underline{\text { 4.2.2.3 Generation of HLA-DRA*0101 }}$}

We amplified the cDNA with the following set of primers, adding an ApaI restriction site to link it to the HLA-DRB1*1501 construct, but also an XhoI at the terminus to link it to the MSCV vector since we removed the synthetic zeta gene.

Forward primer: 5'-AATCCCGGGCCCATGGCCATAAGTGGAGTCCCTGTG ApaI

Reverse primer: 3'-TACTACTCGAGTTATTACAGAGGCCCCCTGCGTTCTGC XhoI

This gene was subcloned into pBS II vector sequenced to confirm it.

\subsubsection{Ligation into the final construct}

Similar to the MBP-DR2- $\zeta$ construct, we chose to subclone leader-MBP with the beta $2 \mathrm{~A}$ gene first to circumvent possible cloning difficulties that might occur in a fourway ligation. The new product, leader-MBP-beta 2A (shown below - 742 bp) was subcloned into the pBS II vector.

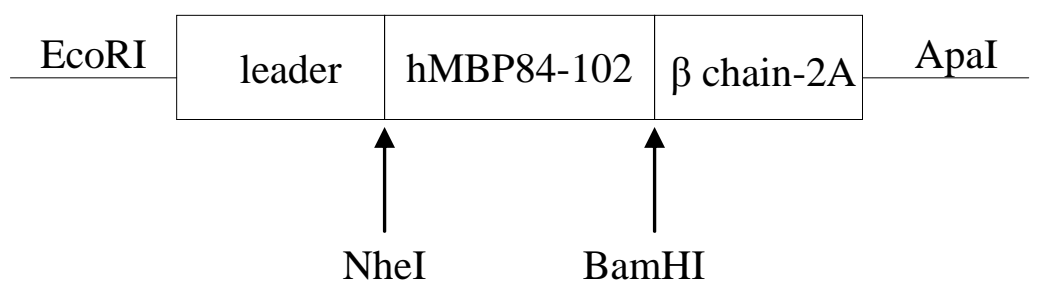

This new gene was then digested with EcoRI and ApaI and then cloned into the final vector, MSCV-IRES-GFP, along with the ApaI/XhoI-cut alpha gene of $736 \mathrm{bp}$, yielding a product of $1.8 \mathrm{~Kb}$. 


\subsubsection{Generation of retrovirus-producing cell lines}

Retroviruses are small oncoviruses with an outer envelope and an inner core containing negative double-stranded RNA responsible for genetic information. Since retroviruses can infect living cells and insert DNA into the genome, they have been converted into gene delivery systems and are able to transduce a wide variety of cell types from different species. For safety reasons, the retrovirus must be able to infect target cells but not replicate. This has been accomplished by replacing the coding region that gives rise to essential viral proteins with the gene of interest to be transferred. This replication-defective virus cannot make the proteins needed for subsequent rounds of replication, but the retrovirus can still enter into the cells and integrate the viral genome in the DNA of the cell. To produce recombinant retroviruses, the env, gag, and pol proteins (still needed for production of new virus) are provided in trans by separate constructs transfected into retroviral packaging cells.

\subsubsection{Generation of MBP-DR2- $\zeta$ and MBP-DR2 retroviruses}

In order to generate the two retroviral ecotropic vectors we used two helper plasmids encoding encoding the gag-pol and env proteins (pEQ.PAM-E and pVSVg) and a plasmid containing the MSCV retroviral vector including the CR constructs. We used 293 T cells as packaging cells for an initial transient transfection. The virus supernatant produced by these cells was then used to infect GP+E86 cells, which were maintained as stable viral producers and from which retrovirus used to transfect T-cells was harvested. We followed the following protocol (75):

Materials: 
293 T cells and GP+E86 cells

Dulbecco's modified Eagle's medium (DMEM) supplemented with $10 \%$ fetal calf serum (FCS) and penicillin-streptomycin-glutamine (PSG) 1\%

pEQ.PAM-E plasmid

pVSVg plasmid

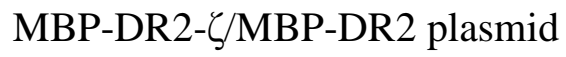

Calcium chloride $\mathrm{CaCl}_{2} 2.5 \mathrm{M}$

2X Hepes Phosphate Buffered Saline (HPBS) pH 7.12

Method:

24-hours prior to transfection, $2 \times 10^{6}$ (or $4 \times 10^{6}$ the day of transfection) $293 \mathrm{~T}$ cells were plated on a $10 \mathrm{ml}$ tissue-culture treated Petri dish. On the day of transfection, four hours prior to the procedure, the medium was changed with fresh complete DMEM. At the time of transfection, cells were approximately $70 \%$ confluent. DNA mix was prepared using $4 \mu \mathrm{g}$ pEQ.PAM-E and $2 \mu \mathrm{g}$ pVSVg and $4 \mu \mathrm{g}$ MBP-DR2-ร/MBP-DR2 plasmid, 50 $\mu \mathrm{l} 2.5 \mathrm{M} \mathrm{CaCl}_{2}$ and adjusted to $500 \mu \mathrm{l}$ with distilled water at room temperature (RT). This mix was then added to $500 \mu \mathrm{l}$ HPBS dropwise while vortexing and incubated 1 minute at RT. The whole mixture was added onto $293 \mathrm{~T}$ cells and incubated for 16 hours at $37^{\circ} \mathrm{C}$. The next day the plates were washed with prewarmed phosphate buffered saline (PBS) twice and new complete DMEM was added. $3 \times 10^{5} \mathrm{GP}+\mathrm{E} 86$ cells were plated in a $10 \mathrm{ml}$ dish. 24-hours later, the medium from 293T cells was replaced, spun at 3000 rotations per minute (RPM) for 5 minutes in a centifuge with rotor and then the supernatant was used to infect GP+E86 cells twice a day, adding $8 \mu \mathrm{g} / \mathrm{ml}$ polybrene to facilitate binding and entry of the retrovirus. The GP+E86 cell line was used to produce retroviral supernatant 
which was then used to transduce $4 \mathrm{G} 4 \mathrm{~T}$ cell hybridoma and activated primary murine $\mathrm{T}$ cells. It is recommendable that the GP+E86 cells be frozen in freezing mix (EHAA medium with $20 \%$ heat inactivated FCS and $10 \%$ dimethyl sulfoxide (DMSO) and kept at $-80^{\circ} \mathrm{C}$ as soon as the transfection has been finished. Since they are stably transfected with retrovirus, they can be thawed out and frozen again multiple times. After thawing, GP+E86 cells were washed once in HBSS medium and plated onto large or medium tissue culture-treated flasks. Retroviral supernatant was collected daily and the medium was replaced until the cells were completely confluent.

\subsubsection{Retroviral transduction of 4G4 T-cell hybridoma}

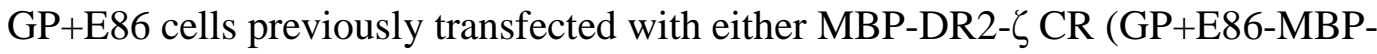
DR2- $\zeta)$ or MBP-DR2 CR (GP+E86-MBP-DR2) were thawed out from the liquid nitrogen tank, washed once with 1x Hanks' balanced salt solution (HBSS, Gibco, Carlsbad, CA), and cultured in 75 or $150 \mathrm{ml}$ culture-treated flanks. Viral supernatant was collected at 24 hours and spun at $3000 \mathrm{rpm}$ for 5 minutes. $4 \times 10^{6} 4 \mathrm{G} 4 \mathrm{~T}$-cell hybridoma were added per well in a 6-well plate and $2 \mathrm{mls}$ of retroviral supernatant and $8 \mu \mathrm{g} / \mathrm{ml}$ polybrene were added, and the cells were spun at $1800 \mathrm{rpm}$ for 90 minutes in a centrifuge with rotor. The procedure was repeated twice for a better yield of $\mathrm{GFP}^{+}$cells.

\subsubsection{Flow cytometry of retrovirally-transduced cells}

To check for HLA class II expression, transduced 4G4 cells were resuspended in FACS buffer (PBS with $1 \%$ BSA and $0.1 \%$ sodium azide $\left(\mathrm{NaN}_{3}\right.$, Sigma) and incubated 30 min on ice with primary phycoerythrin-conjugated (PE) anti-human HLA-DR 
antibody (Becton Dickinson, Franklin Lakes, NJ) at the concentration recommended by the supplier. The cells were then washed and resuspended in FACS buffer and analyzed using a FACSCalibur (BD Biosciences) and CellQuest software. CR-transduced hybridoma were then flow cytometric sorted for $\mathrm{GFP}^{+}$on a MoFlo high-speed cell sorter (DakoCytomation) (courtesy of Dr. Richard Cross, PhD, St. Jude Children's Research Hospital).

\subsubsection{Retroviral transduction of primary murine T-cells}

Lymph nodes and spleen from C57BL/6 mice or OT-1 mice (transgenic for CD8 ${ }^{+}$ cells) were harvested and processed into a single cell suspension by crushing through a $40-\mu \mathrm{m}$ nylon filter. Red blood cells were lysed with Gey's solution $\left(7.0 \mathrm{~g} / 1 \mathrm{NH}_{4} \mathrm{Cl}, 0.37\right.$ $\mathrm{g} / \mathrm{K} \mathrm{KCl}, 0.3 \mathrm{~g} / 1 \mathrm{Na}_{2} \mathrm{HPO}_{4} .12 \mathrm{H}_{2} \mathrm{O}, 0.024 \mathrm{~g} / 1 \mathrm{KH}_{2} \mathrm{PO}_{4}, 1.0 \mathrm{~g} / \mathrm{l}$ glucose, $10.0 \mathrm{mg} / \mathrm{l}$ phenol red, $8.4 \mathrm{mg} / \mathrm{l} \mathrm{MgCl}_{2} .6 \mathrm{H}_{2} \mathrm{O}, 7.0 \mathrm{mg} / 1 \mathrm{MgSO}_{4} .7 \mathrm{H}_{2} \mathrm{O}, 6.8 \mathrm{mg} / \mathrm{CaCl}_{2}$ and $45 \mathrm{mg} / \mathrm{l} \mathrm{NaHCO} 3$ ) and the lymphocytes were cultured in 6-well plates and stimulated with $2 \mu \mathrm{g} / \mathrm{ml}$ purified mouse anti-CD3 $\varepsilon$ and $2 \mu \mathrm{g} / \mathrm{ml}$ anti-CD28 antibodies (Pharmingen, San Diego, CA) or 2 $\mu \mathrm{g} / \mathrm{ml}$ Concanavalin A (ConA) overnight. After 24 and 48 hours, the media was carefully removed and $2 \mathrm{mls} /$ well of viral supernatant was added along with $8 \mu \mathrm{g} / \mathrm{ml}$ polybrene and sorted for GFP expression by flow cytometry. GFP ${ }^{+}$cells were re-fed and split into 6well plates when they reached a high density. They were also sorted for $\mathrm{GFP}^{+}$and on day 10-12, the cells were restimulated with $2 \mu \mathrm{g} / \mathrm{ml}$ ConA and irradiated (4,000 rad) syngeneic splenocytes. 


\subsection{4 $\mathrm{Ob}$ and $6 \mathrm{~F} 11$ target hybridoma cell lines}

\subsubsection{Stimulation of Ob hybridoma with hMBP84-102 peptide}

$10^{5} \mathrm{Ob}$ and $6 \mathrm{~F} 11$ cells were incubated overnight with four different concentrations of purified hMBP84-102 peptide on a 96-well round-bottom plate in complete Bruff medium (EHAA or Click's medium enriched with $10 \%$ FCS, $1 \%$ Penicillin-Streptomycin-Glutamine (PSG), and $4.2 \mu 1$ 2-mercaptoethanol per 1,000 ml medium). Irradiated (20, $000 \mathrm{rad)} \mathrm{MGAR} \mathrm{cells} \mathrm{were} \mathrm{used} \mathrm{as} \mathrm{antigen} \mathrm{presenting} \mathrm{cells.} \mathrm{24-}$ hours later, $60 \mu \mathrm{l}$ of supernatant was removed and submitted for IL-2 secretion analysis by Multiplex (Bio-Rad, Hercules, CA).

\subsubsection{Stimulation of Ob hybridoma with mouse anti-humanV $\beta 2$ antibody}

A 96-well flat bottom plate was coated with $10 \mu \mathrm{g} / \mathrm{ml}$ purified rat anti-mouse IgG (Jackson Immunoresearch Laboratories, Inc., West Grove, PA) and incubated at $4^{\circ} \mathrm{C}$ overnight. 24-hours later, the plate was washed three times with HBSS solution and coated with $50 \mu \mathrm{g} / \mathrm{ml}$ mouse anti-human TCR V $\beta 2$ monoclonal antibody (Immunotech, Warrenale, PA) either overnight at $4^{\circ} \mathrm{C}$ or for two hours at $37^{\circ} \mathrm{C}$. The plate was washed again and $10^{5} \mathrm{Ob}$ or $6 \mathrm{~F} 11$ cells were added. 24-hours later $60 \mu \mathrm{l}$ of supernatant was removed and submitted for IL-2 secretion. 


\subsubsection{Stimulation of TCR/DR2 T lymphocytes}

\subsubsection{Stimulation of TCR/DR2 T lymphocytes with hMBP84-102 peptide}

$5 \times 10^{5}$ cells from lymph nodes or splenocytes of double transgenic mice were cultured in 96-well flat-bottom plates without antigenic peptide or with peptide in different concentrations and irradiated splenocytes from humanized DR2 transgenic mice (denoted as Fug5). After 72 hours of culture, the cells were pulsed with $1 \mu \mathrm{Ci}$ of $\left[{ }^{3} \mathrm{H}\right]$ thymidine and harvested on filtermats 16 hours later. All samples were analyzed in triplicates.

\subsubsection{Stimulation of TCR/DR2 T lymphocytes with EL4-MBP-DR2 cells}

$2 \times 10^{6} \mathrm{~T}$-cells from lymph nodes or splenocytes of transgenic mice were stimulated with $4 \times 10^{6}$ irradiated (20,000 rad) EL4-MBP-DR2 antigen-presenting cells (EL4 thymoma cells transduced with the tailless MBP-DR2 construct) and the same number of irradiated feeders. The cells were grown in complete Bruff medium with $10 \mathrm{U} / \mathrm{ml}$ recombinant IL-2 and split every other day. We have also used this procedure to build an antigen-specific cell line by re-stimulating them in the same conditions on day ten, using Con $\mathrm{A}$ as a mitogen.

\subsubsection{Stimulation of RMTC with HLA-DRB antibody}

The plate-bound antibody stimulation was set up as described at 4.2.4.2. Briefly, transduced 4G4 RMTC were stimulated with three different concentrations of platebound mouse anti-human HLA-DR2 antibody or control goat anti-mouse IgG. 24-hours 
later, $60 \mu \mathrm{l}$ of supernatant was removed and submitted for IL-2 secretion analysis by Multiplex.

\subsubsection{IL-2 secretion by RMTC in response to recognition of Ob hybridoma}

$5 \times 10^{5} 4 \mathrm{G} 4$ hybridoma transduced with MSCV and MBP-DR2- $\zeta$ were cocultured in three different ratios with $\mathrm{Ob}$ or 6F11 target hybridoma in a 96-well round bottom plate for 8 hours. The cells were spun, the medium was replaced, and Brefeldin A (BFA, Epicentre Technologies) was added for 6 hours at $37^{\circ} \mathrm{C}$ and $5-10 \% \mathrm{CO}_{2}$. The cells were spun again at $4^{\circ} \mathrm{C}$ and then washed with staining wash buffer (SWB; 1x PBS, 2\% FBS, $0.1 \% \mathrm{NaN}_{3}$ ) and BFA $10 \mu \mathrm{g} / \mathrm{ml}$. The primary surface antibody (mouse anti-human $\mathrm{V} \beta 2$ PE-conjugated) diluted in SWB+BFA was added and the plate was then incubated on ice for 20 minutes, then washed with PBS+BFA twice and resuspended in the same mix. The cells were then fixed with 2\% formaldehyde in PBS for 20 minutes at RT, washed and incubated in permeabilization buffer (SWB $+0.5 \%$ saponin) for 10 minutes at RT. The cells were then incubated with the APC (Allophycocyanin)-conjugated rat anti-mouse IL2 monoclonal antibody (Becton Dickinson) for 30 minutes on ice, followed by a wash with PBS, and resuspension in SWB. Flow cytometric analysis was performed after gating on the double positive, $\mathrm{APC}^{+}$(on FL4 axis)/GFP (on FL1 axis) cells or alternately, on $\mathrm{APC}^{+}$(on FL4 axis)/Vß2 ${ }^{+}$(on FL2 axis) cells. This way we can identify the cells that secrete IL-2 (featured by APC staining) by gating on either FL1/FL4 (GFP ${ }^{+}$ cells secreting IL-2) or FL2/FL4 (Ob hybridoma secreting IL-2). 


\subsubsection{IFN-y secretion by RMTC in response to recognition of Ob hybridoma}

$5 \times 10^{5} \mathrm{Ob}$ hMBP84-102-specific hybridoma or 6F11 control cells were cocultured with RMTC transduced with MSCV, MBP-DR2- $\zeta$, and MBP-DR2 chimeric receptors for 24 hours. $60 \mu \mathrm{l}$ of supernatant was then removed and submitted for cytokine secretion analysis by Multiplex.

\subsubsection{Proliferation of RMTC in response to stimulation by Ob hybridoma}

$5 \times 10^{4} \mathrm{GFP}^{+}$-sorted $\mathrm{CD} 8^{+}$-transduced RMTC cells were incubated with different ratios of irradiated $\mathrm{Ob}$ or $6 \mathrm{~F} 11$ hybridoma and syngeneic feeders on a 96-flat bottom plate. After 72 hours, the cells were pulsed with $1 \mu \mathrm{Ci}\left[{ }^{3} \mathrm{H}\right]$ thymidine and harvested after 16 hours. Proliferation was measured by liquid scintillation counting of incorporated $\left[{ }^{3} \mathrm{H}\right]$ thymidine. The samples were analyzed in duplicates and plotted as mean \pm 1 stdev.

\subsubsection{Cytolysis of target cells by RMTC}

\subsubsection{Cytolysis of Ob target hybridoma by RMTC assayed by radioactive chromium}

\section{$\underline{(\mathrm{Cr}) \text { release }}$}

$4 \times 10^{6}$ Ob hybridoma specific for human MBP84-102 peptide or 6F11 control hybridoma specific for MBP89-101 were incubated with $200 \mu \mathrm{Ci}{ }^{51} \mathrm{Cr}$ for 30 minutes at $37^{\circ} \mathrm{C}$ with intermittent shaking. After half an hour, the cells were washed three times with PBS saline solution and cocultured in different effector to target ratios with $\mathrm{CD} 8^{+}-\mathrm{RMTC}$

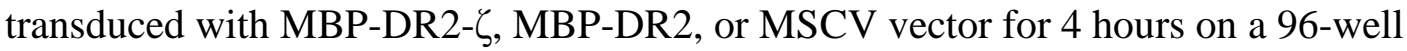
round bottom plate. All cell combinations were set up as triplicates. Target cells in 
medium only were also set for controls. Labeled antigenic targets are recognized and lysed by RMTC, releasing radioactivity into the supernatant. $20 \mu \mathrm{l}$ TritonX (SigmaAldrich, St Louis, MO) was added to ${ }^{51} \mathrm{Cr}$-labeled Ob hybridoma (control wells) and served as a positive control for maximal release of radioactive $\mathrm{Cr}$. Spontaneous lysis (control ${ }^{51} \mathrm{Cr}$ ) was estimated by the release of radioactive $\mathrm{Cr}$ by $\mathrm{Ob}$ hybridoma alone.

After incubation at $37^{\circ} \mathrm{C}$, the plate was centrifuged and $100 \mu \mathrm{L}$ supernatant was harvested and counted. We used the formula below to calculate the percent of specific cytolysis from the release of ${ }^{51} \mathrm{Cr}$ :

$$
\text { (Experimental } \left.{ }^{51} \mathrm{Cr}-\text { control }{ }^{51} \mathrm{Cr}\right) /\left(\operatorname{maximal}{ }^{51} \mathrm{Cr}-\text { control }{ }^{51} \mathrm{Cr}\right) \times 100
$$

\subsubsection{In vitro cytolysis of primary MBP-DR2-specific cells by RMTC}

$10^{5}$ T-cells/well from TCRxDR2 double transgenic (DTG) or TCRxDR2xhCD4 triple transgenic (TTG) mice were cocultured with $\mathrm{CD}^{+} \mathrm{RMTC}$ at different ratios overnight in a 96-well round bottom plate. The next day, the plate was centrifuged and cells were double stained with the mouse anti-human V $\beta 2.1$ antibody (PE) and rat antimouse $\mathrm{CD}^{+}$or $\mathrm{CD}^{+}$antibody (APC). After 30 minutes incubation on ice, the cells were washed and resuspended in SWB. In order to find out the absolute number of target cells left after overnight cytolysis, we used TruCOUNT assay and added TruCount high control fluorescent beads (BD Bioscience, San Jose, CA) at a final concentration of 2000 beads/sample. These beads were used as markers to guarantee that equal fractions of each sample were enumerated, thus allowing quantitative comparison of the number of events and eliminating the variability between samples. The absolute number of cells in the sample can be determined by normalizing to the number of acquired beads. 
FACSCalibur $^{\mathrm{TM}}$ was used with forward (FCS) and side scatter (SSC) set in a logarithmic mode. TruCount beads were gated in a FCS/SSC plot and analysis was stopped when 500 beads were counted. We then ran the sample tubes and analyzed the double positive $\mathrm{hV} \beta 2.1^{+} \mathrm{CD} 4^{+}$or $\mathrm{hV} \beta 2.1^{+} \mathrm{CD} 8^{+}$cells after gating and counting 500 beads.

\subsubsection{In vitro cytolysis of primary MBP-DR2-specific T-cells by RMTC detected by} $\underline{\text { stimulation with EL4-MBP-DR2 cells }}$

This experiment was set up similarly to 4.2.10.2. Unlike the previous experiment, we chose not to directly analyze the residual cells after the overnight coculture of the two cell types, but instead we added $2 \times 10^{5}$ EL4-MBP-DR2 antigen-presenting cells or control EL4 cells at the time of the culture, for five days. On day five we stained each well with mouse anti-human V $\beta 2.1$ and rat anti-mouse $\mathrm{CD} 4^{+}$and $\mathrm{CD}^{+}$antibodies and analyzed the residual double positive cell counts by quantitative flow cytometry.

\subsubsection{Cytolysis of target DTG cell lines by RMTC}

$10^{5}$ double or triple transgenic cell lines (obtained as described in 4.2.5.2) were cocultured with MSCV retroviral vector control, MBP-DR2- $\zeta$, or MBP-DR2 RMTC in a 96-well round bottom plate for six hours at four different ratios. After 6 hours, we stained the cells as described in 4.2.10.2 and evaluated the number of residual $\mathrm{hV} \beta 2.1^{+} \mathrm{CD} 4^{+}$and $\mathrm{hV} \beta 2.1^{+} \mathrm{CD}^{+}$cells by quantitative FACS. 


\subsection{Results}

\subsubsection{Design of chimeric receptors}

In order to specifically target pathologic, hMBP-specific T lymphocytes, we engineered two chimeric receptors: MPB-DR2- $\zeta$ and MBP-DR2. The first CR included the extracellular and transmembrane domains of the human MHC class II beta chain DRB $1 * 1501$ linked to the human MBP peptide epitope $84-102$ and the $\zeta$ cytoplasmic domain of the TCR. A $2 \mathrm{~A}$ sequence from $\mathrm{TaV}$ connected this part of the construct with the human MHC class II alpha chain DRA*0101 paired with the $\zeta$ cytoplasmic tail of TCR. The whole gene was subcloned in MSCV retroviral expression vector that contains an IRES site and the GFP gene (Figure 4-1). In parallel we designed a tail-deficient CR that lacked any signaling moiety from the TCR (Figure 4-2).

\subsubsection{Transfection of CRs into transient and permanent viral-producing cell lines}

Transfection of both retroviruses had a good yield in both 293T and GP+E86 cells (Figures 4-3 and 4-4). The latter cell line was used to produce retroviral supernatant which was then used to transduce 4G4 T-cell hybridoma and activated murine T-cells.

\subsubsection{Transduction of 4G4 T-cell hybridoma and primary murine T-cells}

Transduction of both 4G4 T-cell hybridoma and primary murine T-cells had a good yield, although we noticed a difference between the efficiency of transduction in 4G4 hybridoma and primary $\mathrm{T}$ lymphocytes. $4 \mathrm{G} 4$ cells transduced with the $\zeta$-bearing $\mathrm{C}$ showed an equivalent surface expression of the receptor compared to the $\zeta$-deficient $\mathrm{CR}$, 


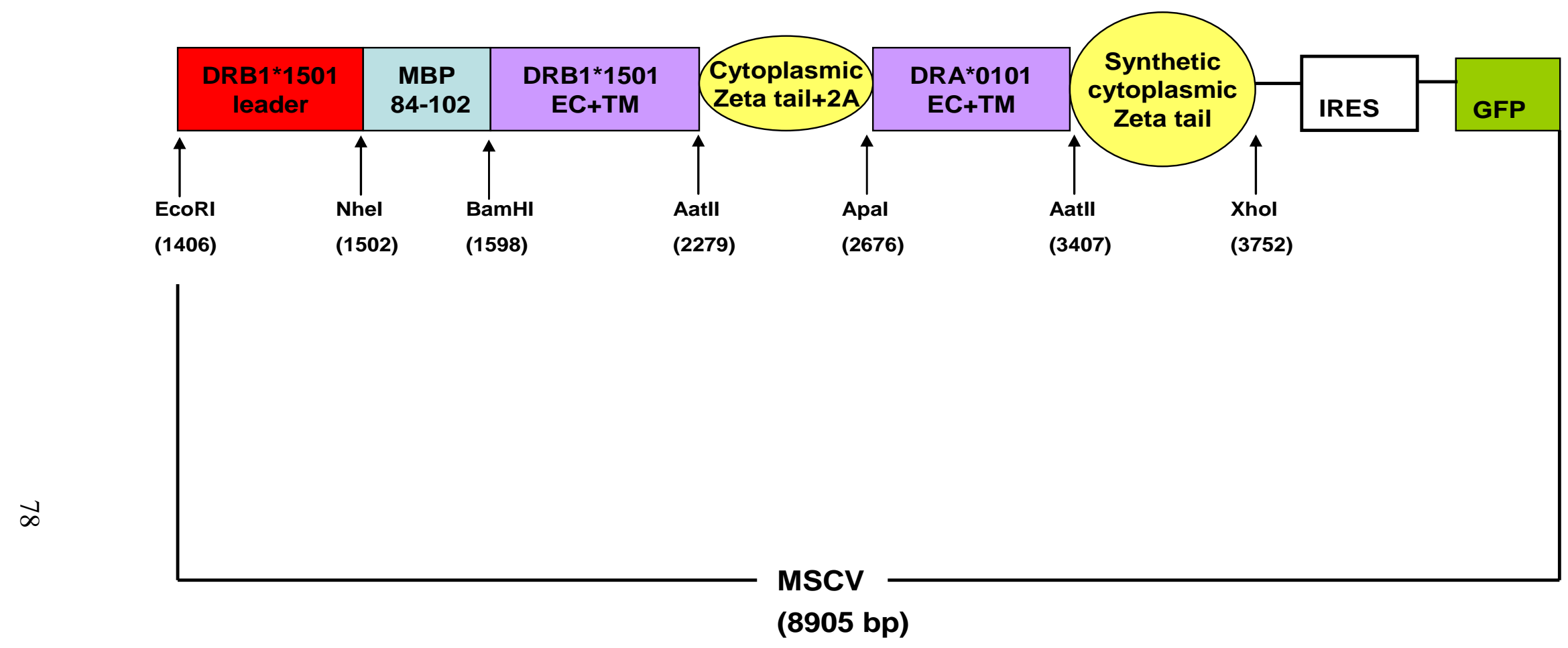

Figure 4-1. MSCVII-GFP-MBP-DR2- $\zeta$ construct. The immunodominant epitope of the human myelin basic protein was genetically linked to the extracellular and transmembrane domains of MHC class II $\beta$ chain chain and human cytoplasmic TCR $\zeta$ chain. This was then paired with the extracellular and transmembrane domains of MHC class II $\alpha$ chain and another cytoplasmic signaling tail. The 2A sequence from FMDV was inserted between the DRB $\beta$ and $\alpha$ chain to provide equal amounts of the bicistronic message. 


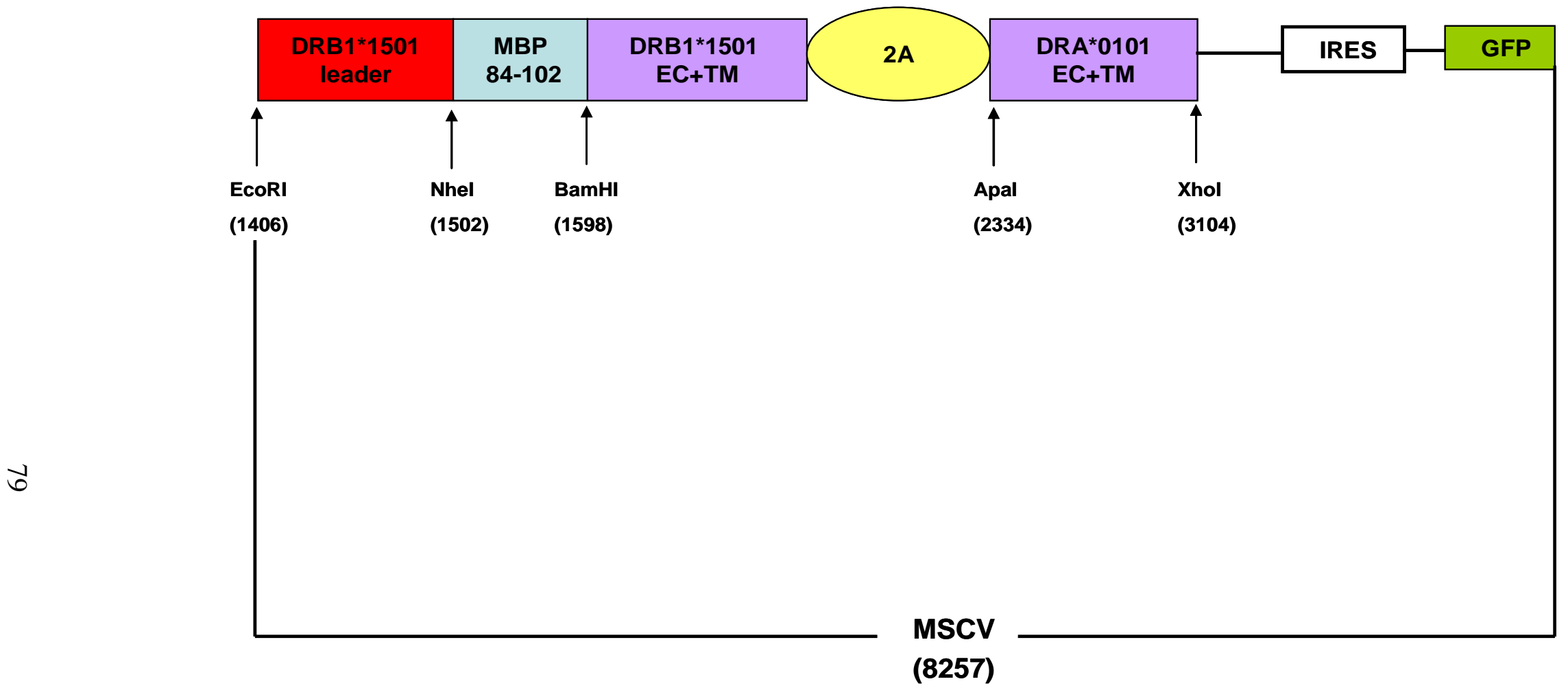

Figure 4-2. MSCV II-GFP-MBP-DR2 construct. This construct is similar to MSCV II-GFP-MBP-DR2- $\zeta$ construct (in Figure 4-1) with the exception of the $\zeta$ cytoplasmic signaling tail. 

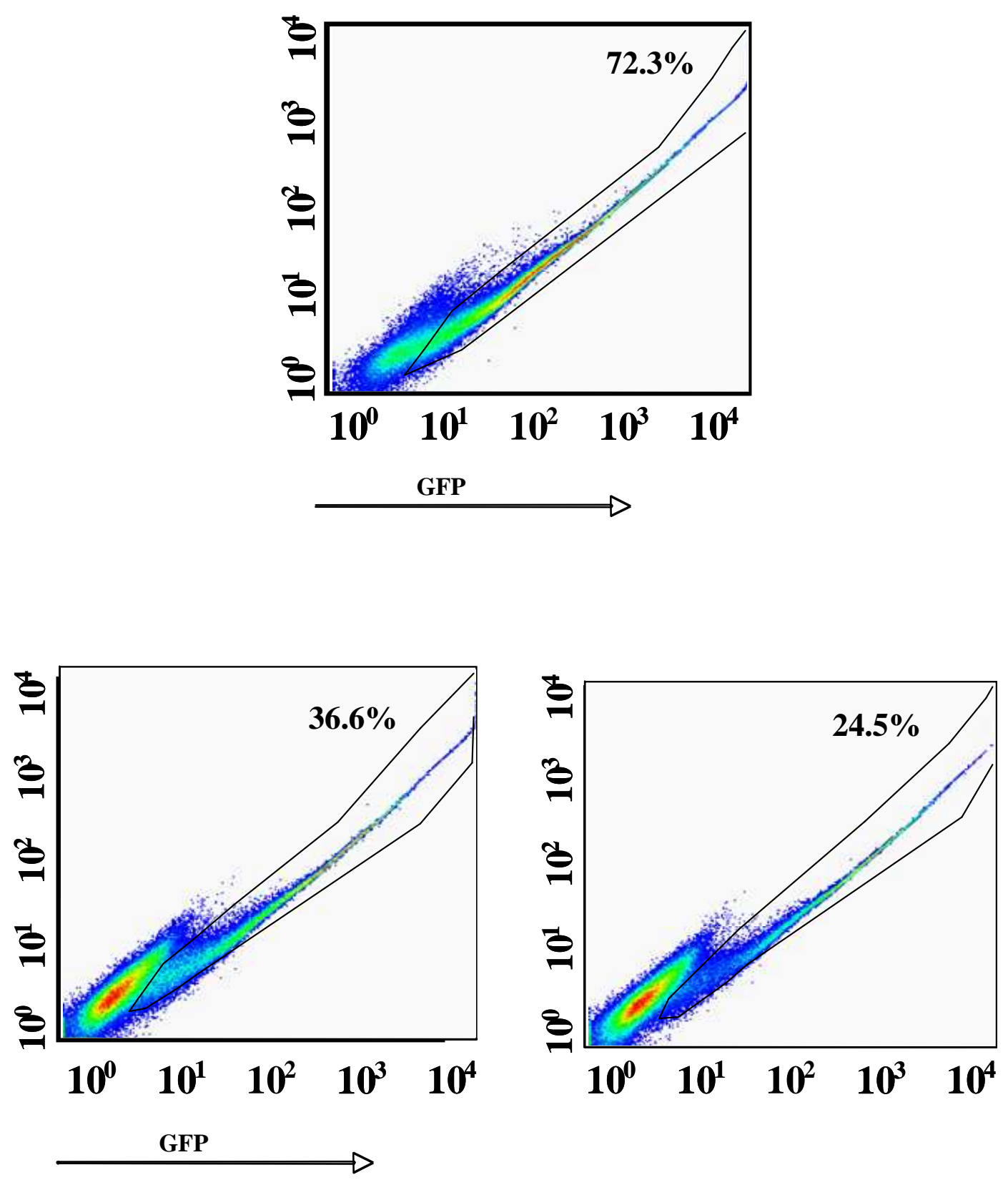

Figure 4-3. Transient retroviral transfection in 293T cells. $293 \mathrm{~T}$ cells were transiently transfected with retrovirus according to the methods. The percentage represents the $\mathrm{GFP}^{+}$ cells. FL1 axis shows uncompensated $\mathrm{GFP}^{+}$cells. Top center: 293T cells transfected with MSCV (empty virus). Bottom left: 293T cells transfected with MBP-DR2- $\zeta$ construct. Bottom right: 293T cells transfected with MBP-DR2 construct. 

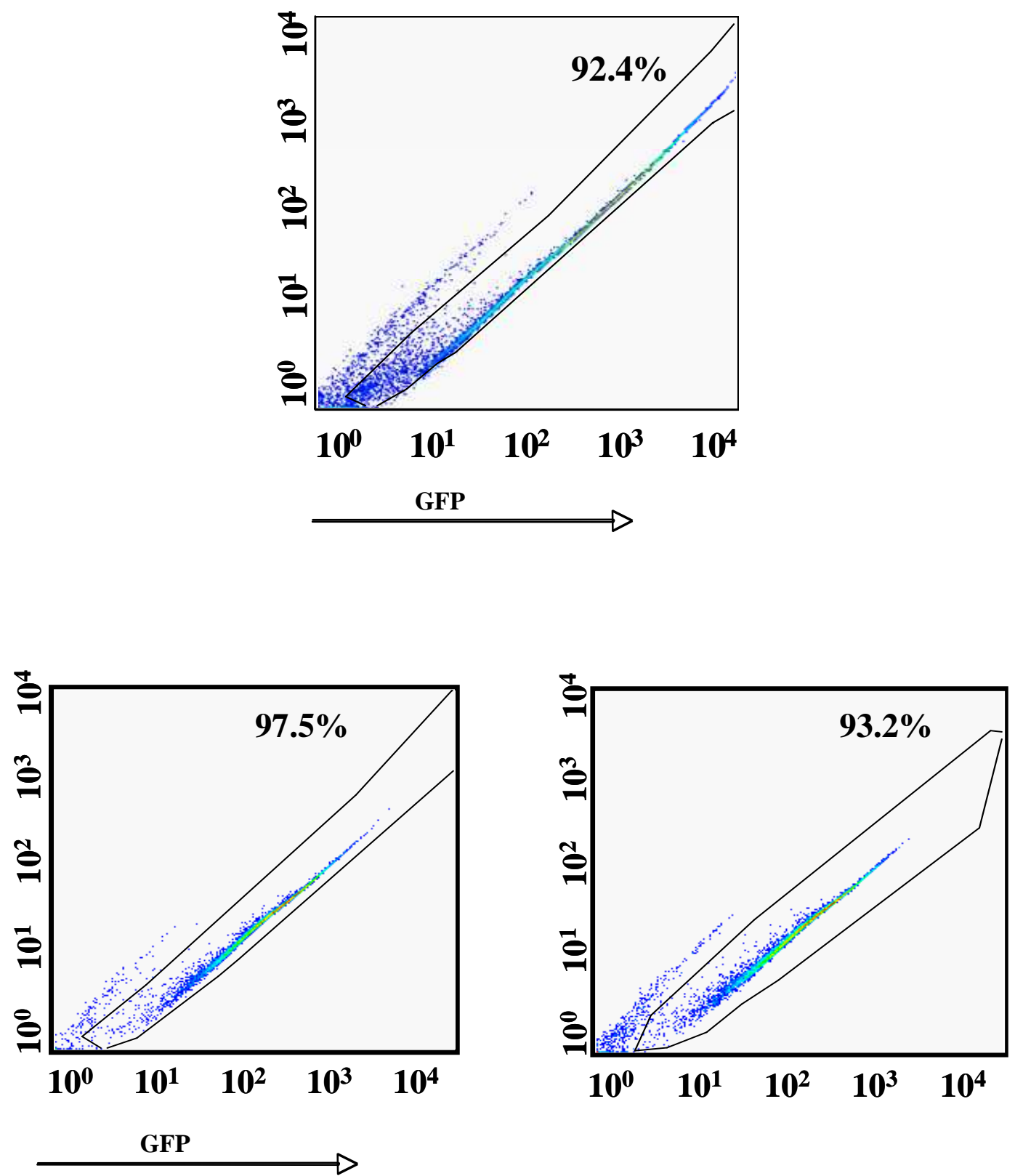

Figure 4-4. Retroviral transfection in GP+E86 cells. GP+E86 cells were transfected with retroviral supernatant from $293 \mathrm{~T}$ cells according to the methods. The percentage represents the $\mathrm{GFP}^{+}$cells. FL1 axis shows uncompensated $\mathrm{GFP}^{+}$cells. Top center: GP+E cells transfected with MSCV (empty virus). Bottom left:GP+E cells transfected with MBP-DR2- $\zeta$ construct. Bottom right: GP+E cells transfected with MBP-DR2 construct. 
whereas transduction in murine $\mathrm{T}$ lymphocytes showed an approximately $30 \%$ increase in the surface expression levels of MBP-DR2 CR compared with MBP-DR2- $\zeta$ (Figures 4-5 and 4-6).

In conclusion, there's a clear difference between the transduction efficiency in hybridoma compared to activated T-cells. Hybridoma are very uniform, clonally expanded cells that would exhibit the same ability to harbor a retrovirally-transduced gene. The intracytoplasmic signaling tail renders the CR capable of signal transduction and therefore, primary activated T-cells transduced with the tail-bearing CR will internalize the surrogate receptor, leading to a decreased surface expression of the CR. Removal of the signaling tail restores the surface expression of the CR to almost the same levels as MSCV control.

\subsubsection{Specificity of $\mathrm{Ob}$ hybridoma}

Before we proceeded to use $\mathrm{Ob}$ hybridoma as target cell lines, we first needed to ensure their antigenic-specificity by testing their response to stimulation with peptide and the mouse anti-human $\mathrm{V} \beta 2$ antibody.

\subsubsection{Stimulation of Ob hybridoma with hMBP84-102 peptide}

To verify the specificity of $\mathrm{Ob}$ hybridoma for hMBP, we stimulated the cells with different concentrations of antigenic peptide. The Ob hybridoma secreted IL-2 in response to peptide stimulation. The amount of IL-2 secreted by the cells was directly proportional to the amount of antigenic peptide used to stimulate the cells. Control $6 \mathrm{~F} 11$ hybridoma did not show any response to stimulation with hMBP restricted by HLA-DR2 

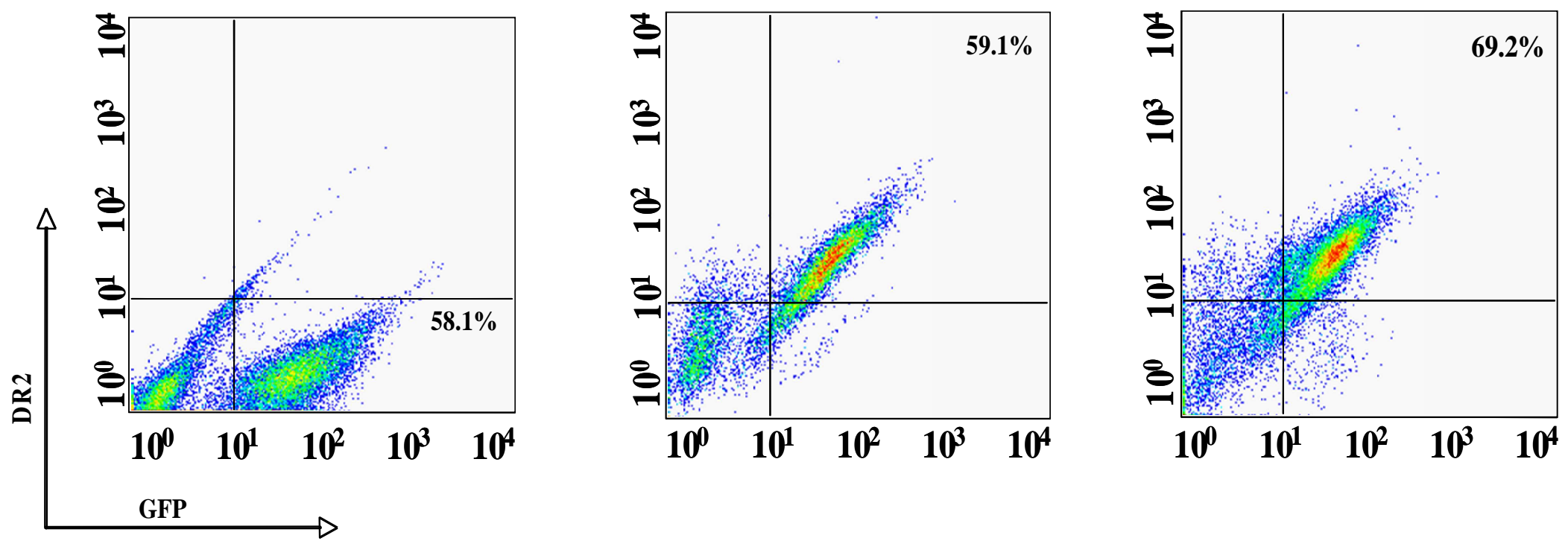

Figure 4-5. Expression of HLA-DR2 on 4G4 T-cell hybridomas transduced with chimeric receptors. MSCV retroviral vector control, $\zeta$-bearing, and $\zeta$-deficient $\mathrm{CRs}$ were transduced into $4 \mathrm{G} 4 \mathrm{TCR}^{-} /$hybridomas. The efficiency of transduction is shown by the amount of GFP which represents the surface expression level of the chimeric receptors. The cells were stained with mouse anti-human HLA-DR2 antibody and then analyzed by FACS. Left panel: MSCV-transduced

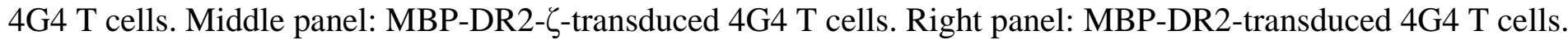



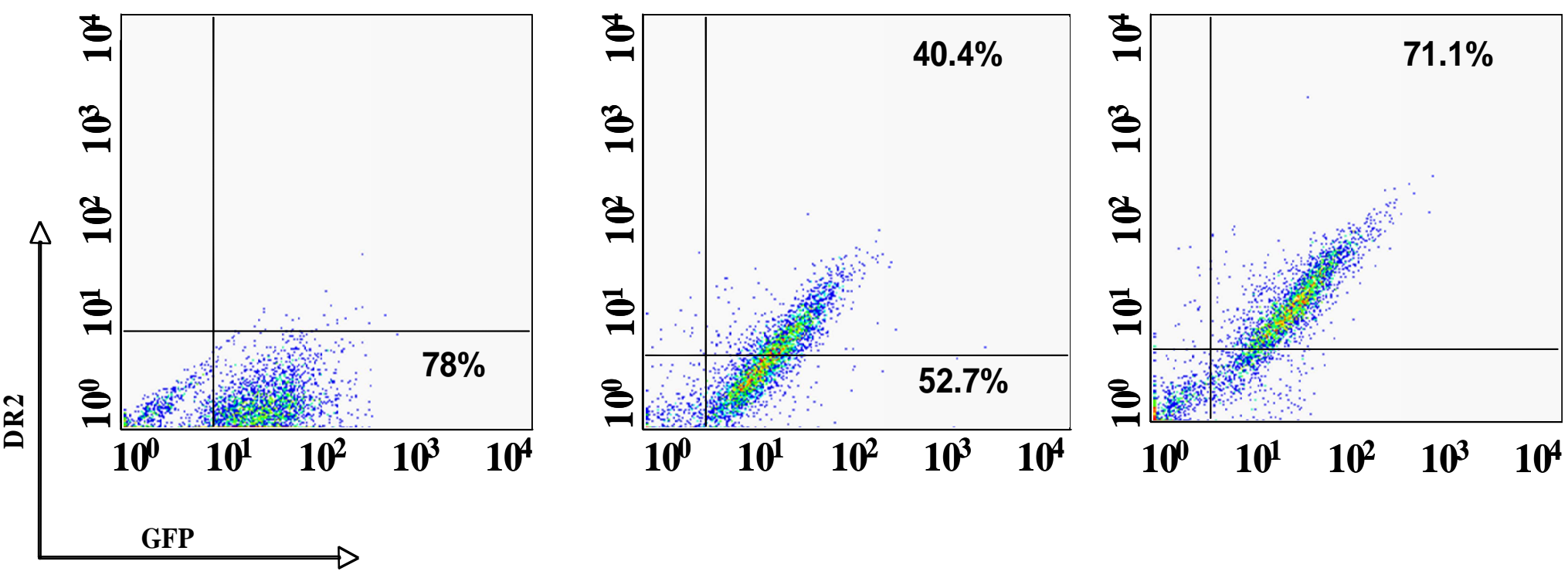

Figure 4-6. Expression of HLA-DR2 on primary murine $\mathrm{CD8}^{+} \mathrm{T}$-cells transduced with chimeric receptors. MSCV retroviral vector control, $\zeta$-bearing, and $\zeta$-deficient CRs were transduced into primary $\mathrm{CD} 8^{+} \mathrm{T}$-cells. The efficiency of transduction is shown by the amount of GFP which represents the surface expression level of the chimeric receptors. The cells were stained with mouse anti-human HLA-DR2 antibody and then analyzed by FACS. Left panel: MSCV-transduced T-cells. Middle panel: MBP-DR2- $\zeta$-transduced T-cells. Right panel: MBP-DR2-transduced T-cells. 
(Figure 4-7 upper panel).

\subsubsection{Stimulation of Ob hybridoma with mouse anti-human $\mathrm{\beta} \beta 2$ antibody}

Ob hybridoma are specific for hMBP84-102 restricted by HLA-DR2 and they stain for hV $\beta 2$ antibody. Upon stimulation with plate-bound mouse anti-human V $\beta 2$, Obspecific, but not $6 \mathrm{~F} 11$ control cells responded to this stimulation by IL-2 secretion (Figure 4-7 lower panel).

In conclusion, we hereby proved that Ob hybridoma constitutes a clonal population of cells specific for hMBP84-102 peptide and they respond stimulation with either the peptide or the specific Vbeta antibody by proliferation.

\subsubsection{Specificity of T lymphocytes from TCR/DR2 transgenic mice}

\subsubsection{Stimulation of TCR/DR2 T lymphocytes with hMBP84-102 peptide}

To reproduce previously published data, we stimulated $\mathrm{T}$ lymphocytes from double transgenic TCRxDR2 mice with hMBP84-102. We did not obtain consistent results, and the proliferative response of T-cells proved extremely variable with different mice and experiments. T-cells removed from the lymph nodes of some of the mice responded very well to peptide stimulation, while others did not (Figure 4-8).

\subsubsection{Stimulation of TCR/DR2 T lymphocytes with EL4-MBP-DR2 cells}

Considering the results from the previous experiment, we searched for alternative ways of stimulating antigen-specific cells from DTG mice. Specifically, because we 

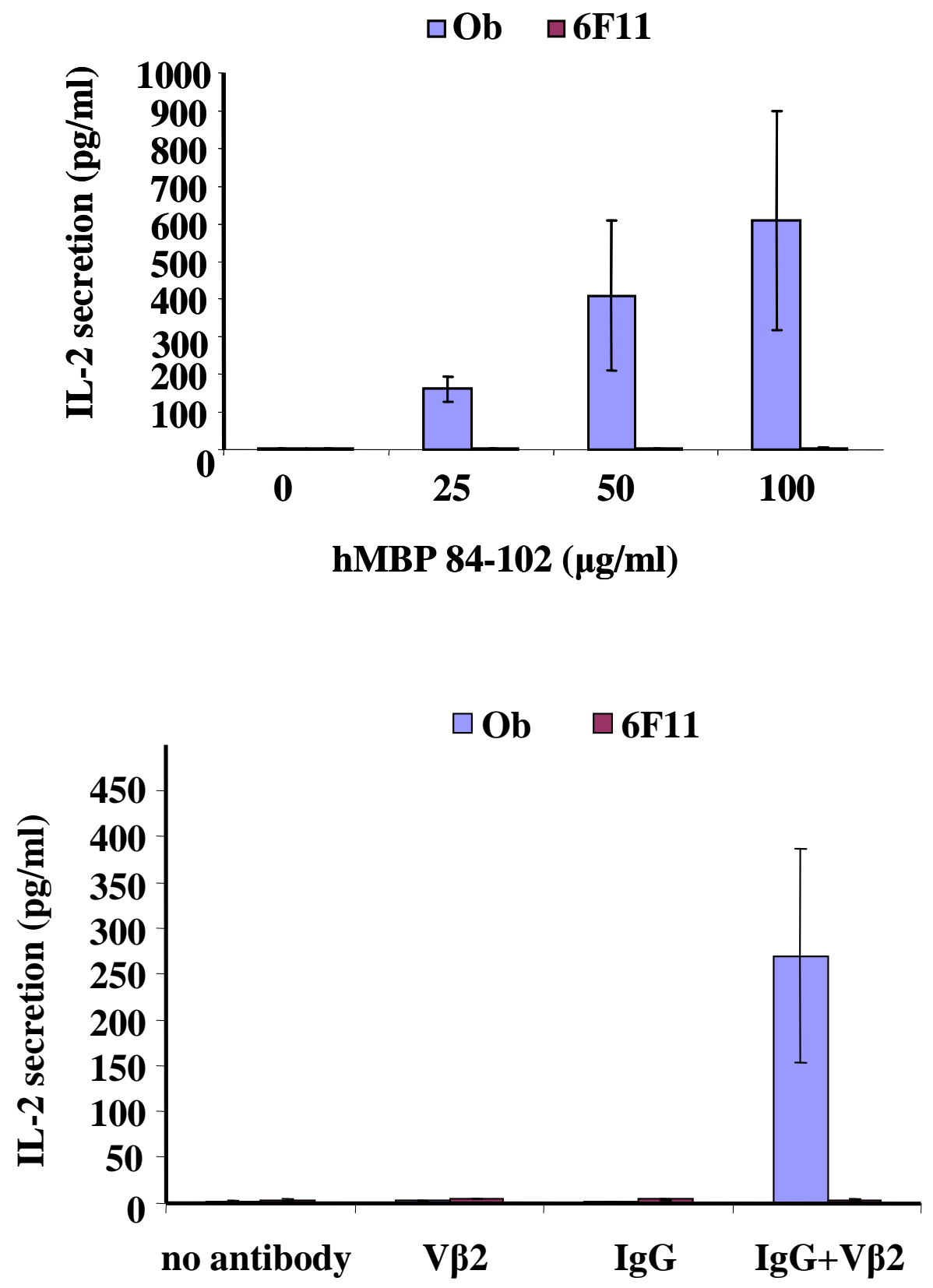

Figure 4-7. Ob cells respond to stimulation by producing IL-2. Ob and control 6F11 hybridoma were stimulated with different dilutions of hMBP peptide (upper panel) or mouse anti-human TCR V $\beta 2$ (lower panel) antibody and secretion of IL-2 was assayed after 24 hours. Only Ob hMBP-specific cells, but not 6F11 control cells responded to stimulation by the secretion of IL-2. 

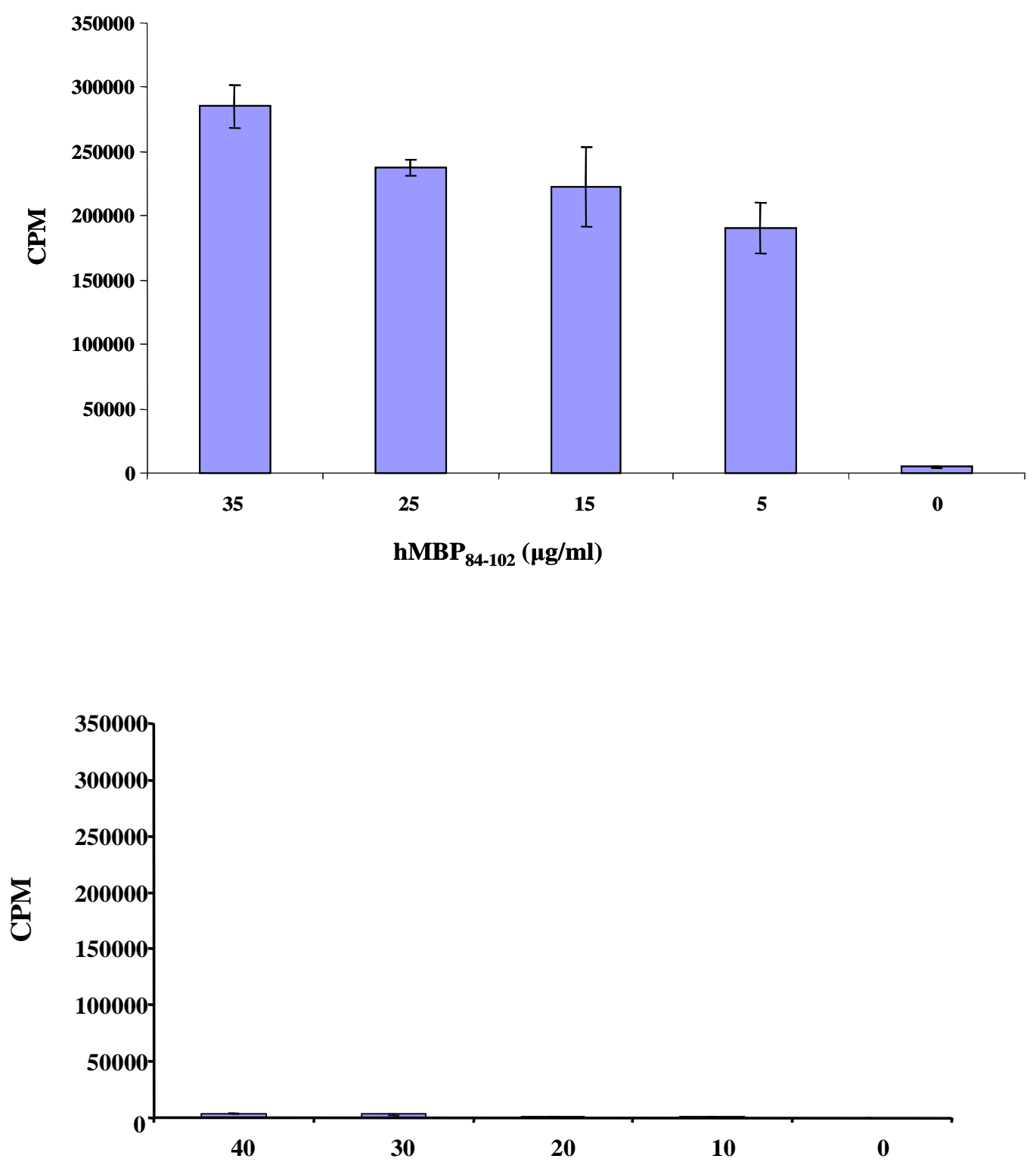

hMBP84-102 $(\mu \mathrm{g} / \mathrm{ml})$

Figure 4-8. Proliferation of TCR/DR2 cells in response to hMBP peptide. T-cells from lymph nodes of TCR/DR2 mice were stimulated with different concentrations of peptide and 72 hours later $1 \mu \mathrm{Ci}\left[{ }^{3} \mathrm{H}\right]$ thymidine was added to each well. Proliferation was measured by liquid scintillation counting. Top figure: in this particular experiment, the transgenic T-cells proliferated robustly in response to peptide stimulation. Bottom figure: in this experiment, the transgenic T-cells did not respond to peptide stimulation. 
suspected that the low expression levels of DR2 gene on B cells from humanized class II transgenic mice (Fug5) were impeding the proper stimulation of hMPB-specific cells, we we tested the use for EL4 thymoma cells transduced with the MBP-DR2 tail-deficient construct as a stimulus. This way the antigen-specific cells would undergo a proper stimulation, but will not suffer the effects that the tail-bearing CR would provide. We showed that EL4-MBP-DR2 cells, though not EL4 cells alone, stimulate lymph node or spleen-derived T-cells from DTG or TTG mice due to expression of hMBP-DR2 (Figure 4-9).

\subsubsection{Stimulation of RMTC by HLA-DRB antibody}

Since RMTC are transduced with CRs containing the DR2 gene, we wanted to first test the ability of our CRs to be stimulated by the specific MHC class II antibody. We used 4G4 T cell hybridoma expressing the CRs. Upon stimulation with the platebound mouse anti-human DR2 antibody, only 4G4-MBP-DR2- $\zeta$ cells responded by IL-2 secretion. This demonstrates that the chimeric receptor bearing the signal transduction domain is the only one able to transmit a functional signal and that deletion of the $\zeta$ cytoplasmic domain impairs this ability (Figure 4-10). 


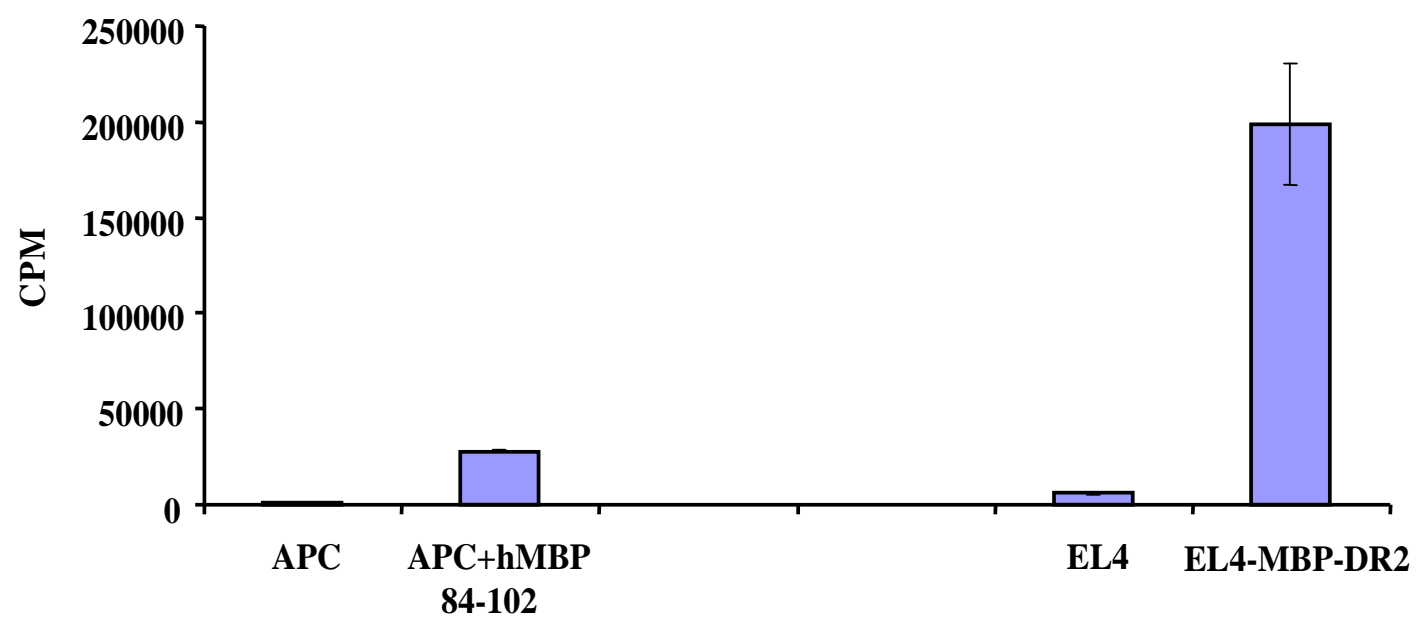

$\square \mathrm{CD8}^{+} \mathrm{ObFug5}$

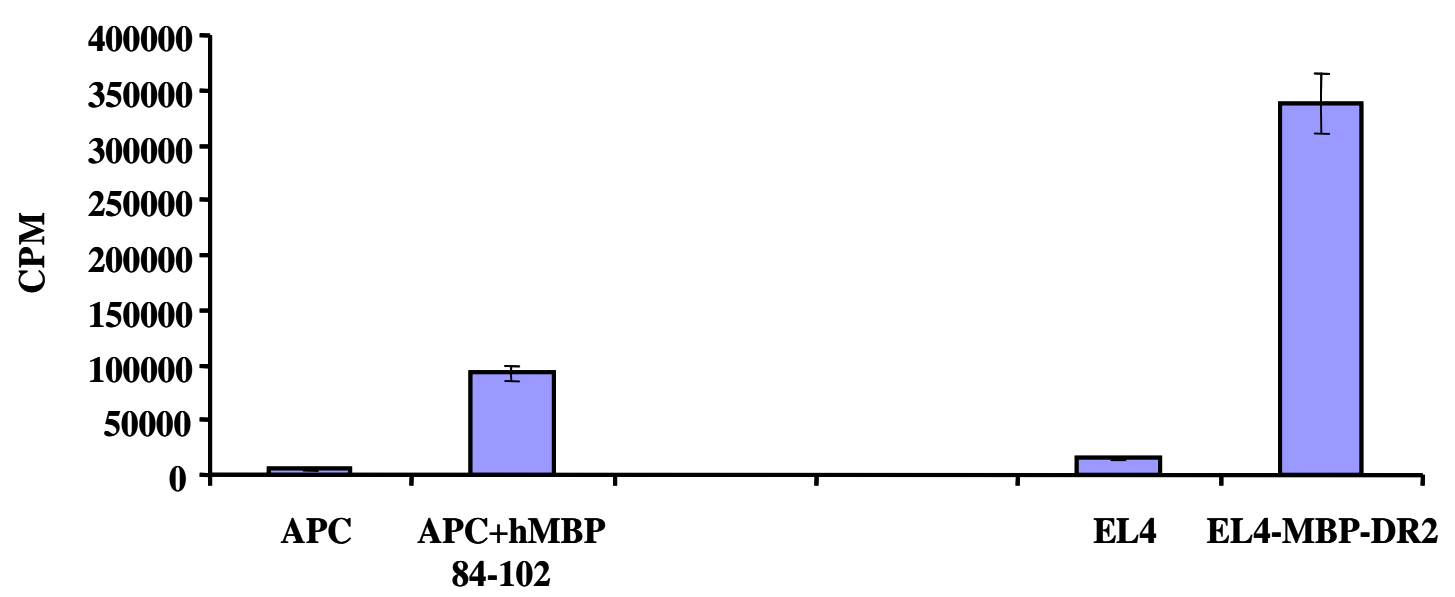

Figure 4-9. $\mathrm{CD4}^{+}$and $\mathrm{CD8}^{+}$TCR/DR2 cells respond to stimulation by EL4-MBPDR2. $\mathrm{CD}^{+}$(top panel) and $\mathrm{CD}^{+}$(bottom panel) cells from lymph nodes and spleens of TCR/DR2 mice were sorted and stimulated with hMBP peptide, EL4 control, or EL4DR2 antigen-presenting cell lines (EL4-MBP-DR2) for 72 hours. Proliferation was assayed by $\left[{ }^{3} \mathrm{H}\right]$ thymidine incorporation. 


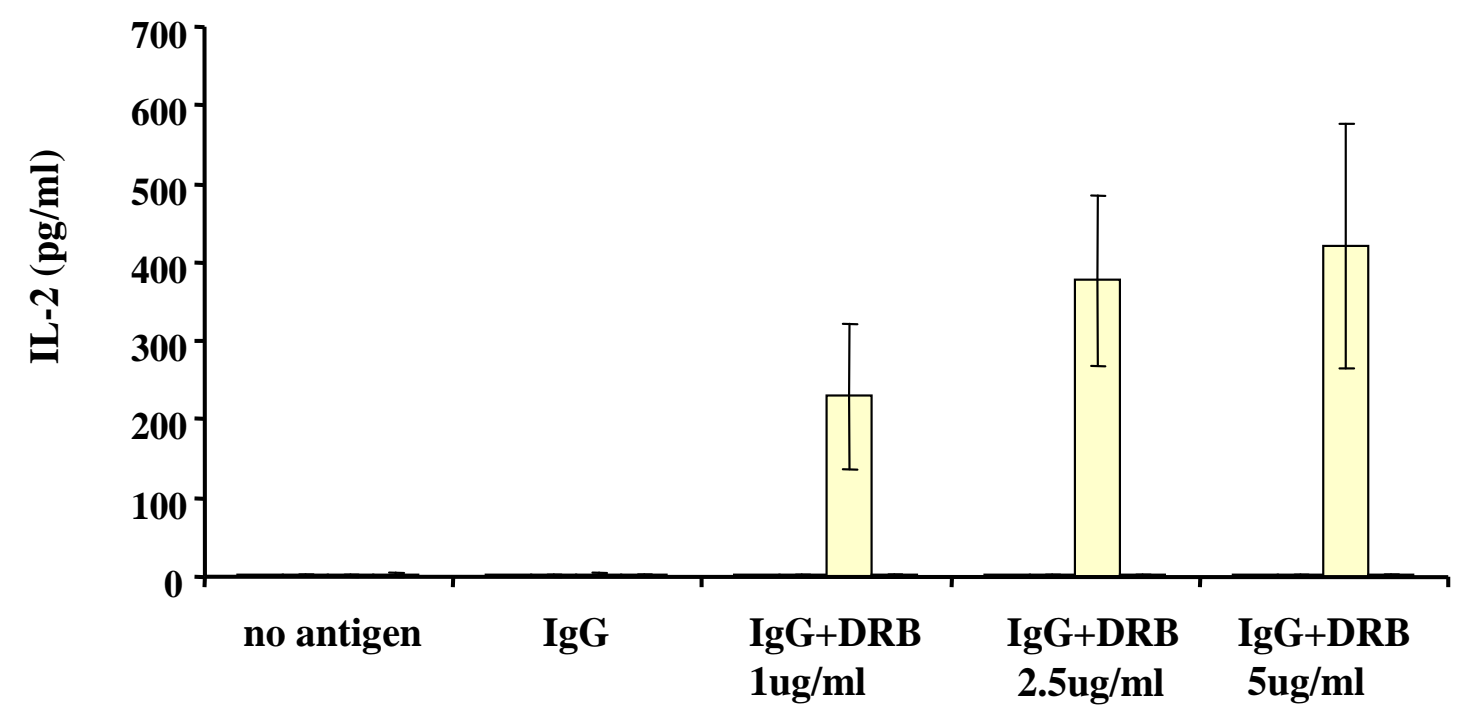

Figure 4-10. Stimulation of RMTC by anti-HLA-DR2 antibody. 4G4 T-cells were transduced with MBP-DR2- $\zeta$, MBP-DR2, and MSCV control retrovirus and cultured overnight on an anti-DR2 antibody-coated plate. 24-hours later, $60 \mu 1$ supernatant was removed and checked for secretion of IL-2. 4G4-MBP-DR2- $\zeta$ cells showed exclusive secretion of IL-2. 


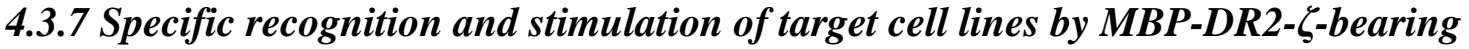 RMTC}

\subsubsection{Recognition of cognate TCR by RMTC}

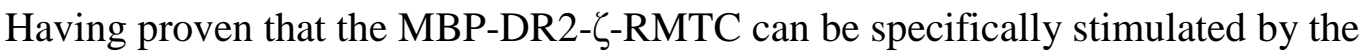
anti-DR2 antibody, we next wanted to test the ability of the RMTC to specifically recognize cognate TCR on the surface of $\mathrm{Ob}$ target hybridoma and transmit a signal in response to this recognition. Upon coculture with irradiated hMBP84-102/DR2-specific Ob hybridoma but not control MBP89-101/I-A ${ }^{\mathrm{s}}$-specific 6F11 hybridoma, MBP-DR2$\zeta$-transduced 4G4 T-cells secreted IL-2 detectable by flow cytometry after intracytoplasmic staining. Although this approach does not provide information about the amount of IL-2 secreted, we were nevertheless able to identify the specific interaction that yielded this cytokine (Ob hybridoma and 4G4-MBP-DR2- $\zeta$ cells) and the specific cell type that produced it (4G4-MBP-DR2- $\zeta$ cells). This was an important piece of information since both the Ob and RMTC cells can produce IL-2 and it proved that the RMTC were specifically responding to cognate TCR stimulation (Figure 4-11).

To analyze similar recognition by $\mathrm{CR}$-transduced primary $\mathrm{CD} 8^{+} \mathrm{T}$-cells, we

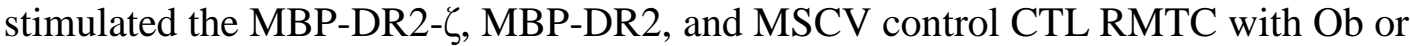
6F11 control and quantitated the secretion of IFN- $\gamma$. Hybridoma do not produce this cytokine, whereas primary T-cells do. This shows that the only cell type producing INF- $\gamma$ would be the tail-bearing RMTC (Figure 4-12). 

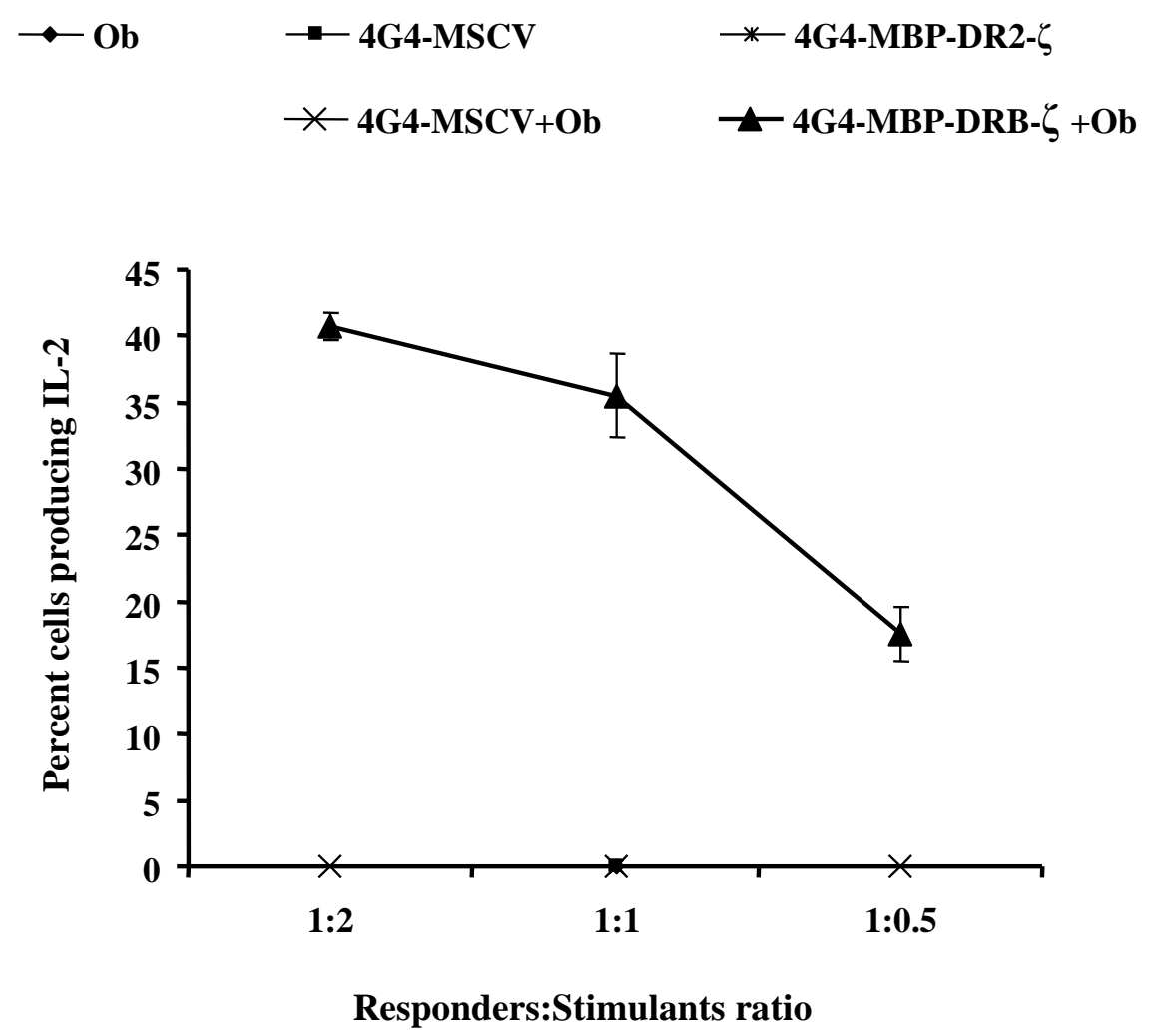

Figure 4-11. Intracellular IL-2 production by 4G4-MBP-DR2- $\zeta$ cells. Ob and $6 \mathrm{~F} 11$ target cells were cocultured with 4G4-RMTC for 8 hours and the number of cells producing IL-2 was assessed by flow cytometry. 4G4 cells transduced with the $\zeta$-bearing chimeric receptor secreted IL-2 in response to stimulation by Ob specific, but not control $6 \mathrm{~F} 11$ hybridoma. 

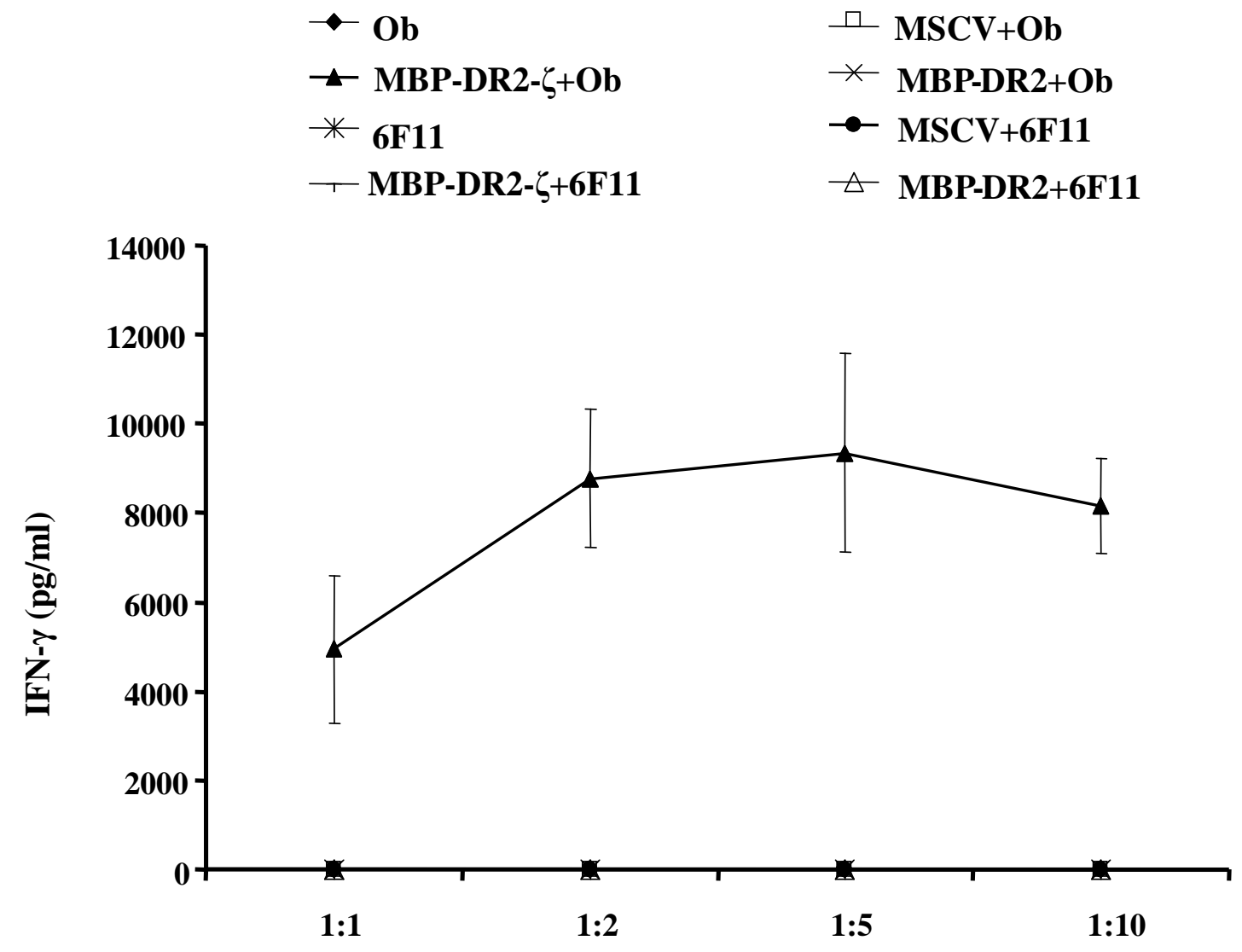

Responders: Stimulants ratio

Figure 4-12. IFN- $\gamma$ secretion by MBP-DR2- $\zeta$ RMTC stimulated by Ob hybridoma. We cocultured target (Ob or 6F11 cells) with RMTC effector cells at 4 different ratios. IFN- $\gamma$ was secreted by MBP-DR2- $\zeta \mathrm{CD}^{+} \mathrm{T}$-cells (but not MBP-DR2 CD8 ${ }^{+}$T-cells or MSCV retroviral vector control cells) upon recognition of cognate TCR on Ob, but not control 6F11 hybridoma. 


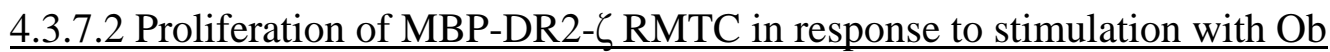

hybridoma

Irradiated $\mathrm{Ob}$ hybridoma were capable of inducing proliferation of $\mathrm{CD} 8^{+} \mathrm{MBP}-$

DR2- $\zeta$ RMTC at all tested ratios of responders to target cells (Figure 4-13). This

experiment, along with the previous ones, proved that RMTC can recognize antigen-

specific T-cells upon cognate TCR-CR interaction and this recognition induces the

RMTC effector functions, including cytokine secretion and proliferation.

\subsubsection{Cytolysis of target cells by RMTC}

The ultimate goal that the RMTC were designed for is the specific cytolysis of antigen-specific cells or cell lines. We used two types of target cells: Ob or 6F11 control hybridoma, and naïve or activated T-cells from TCR/DR2 transgenic mice.

\subsubsection{Cytolysis of Ob target hybridoma by RMTC}

The cytolytic activity of genetically modified $\mathrm{CD} 8^{+} \mathrm{RMTC}$ was evaluated by the release of ${ }^{51} \mathrm{Cr}$ after four hours of coculture of RMTC with ${ }^{51} \mathrm{Cr}$-labeled $\mathrm{Ob}$ or $6 \mathrm{~F} 11$ hybridoma. $\mathrm{CD}^{+}{ }^{+} \mathrm{RMTC}$ bearing the $\zeta$-chimeric receptor were capable of specifically killing over $70 \%$ of antigen-specific target Ob hybridoma, but did not kill 6F11 controls even at effector:target ratio as low as 1:1 cells. Tailless MBP-DR2 RMTC and MSCV control T-cells were not able to kill either targets. This shows that RMTC bearing the $\zeta$ CR are able to specifically and efficiently kill hMBP84-102/DR2 target Ob hybridoma, whereas removal of intracytoplasmic signaling tail renders RMTC unable to perform their killing function (Figure 4-14). 

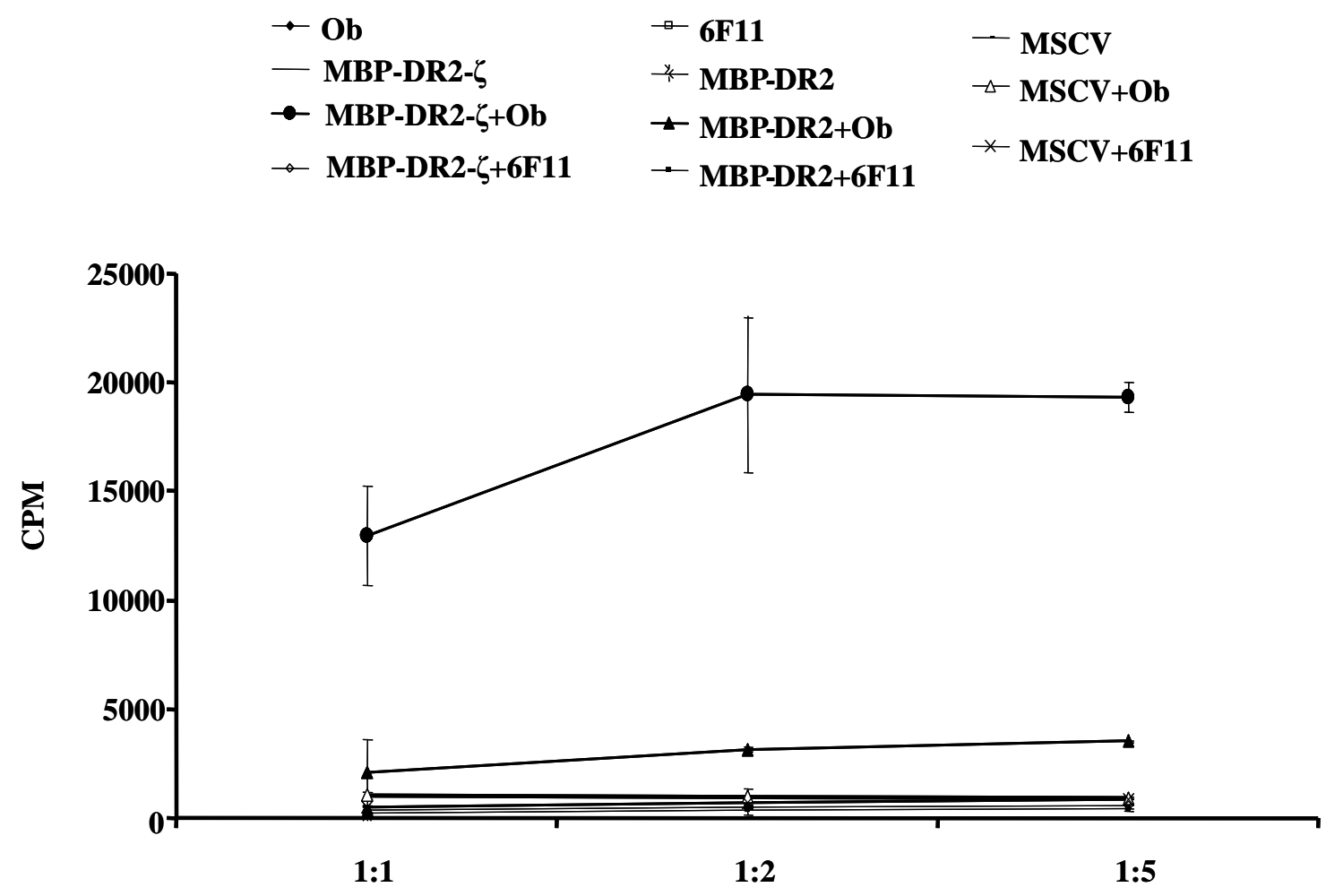

Responders:Stimulants ratio

Figure 4-13. Proliferation of $\mathrm{CD8}^{+} \mathrm{MBP}-\mathrm{DR} 2-\zeta$ RMTC in response to $\mathrm{Ob}$

hybridoma. Irradiated $\mathrm{Ob}$ or control $6 \mathrm{~F} 11$ hybridoma were cocultured in different ratios with $\mathrm{CD}^{+}$RMTC and on day $3,\left[{ }^{3} \mathrm{H}\right]$ thymidine was added and liquid scintillation counting was performed 24 hours later. At any given ratio, Ob hybridoma could only stimulate the proliferation of MBP-DR2- $\zeta$-RMTC, but not the tailless CR-transduced Tcells or MSCV retroviral vector-transduced cells. 


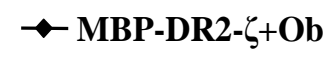

MBP-DR2- $\zeta+6 F 11$ $\rightarrow$ MBP-DR2+Ob

* MBP-DR2+6F11

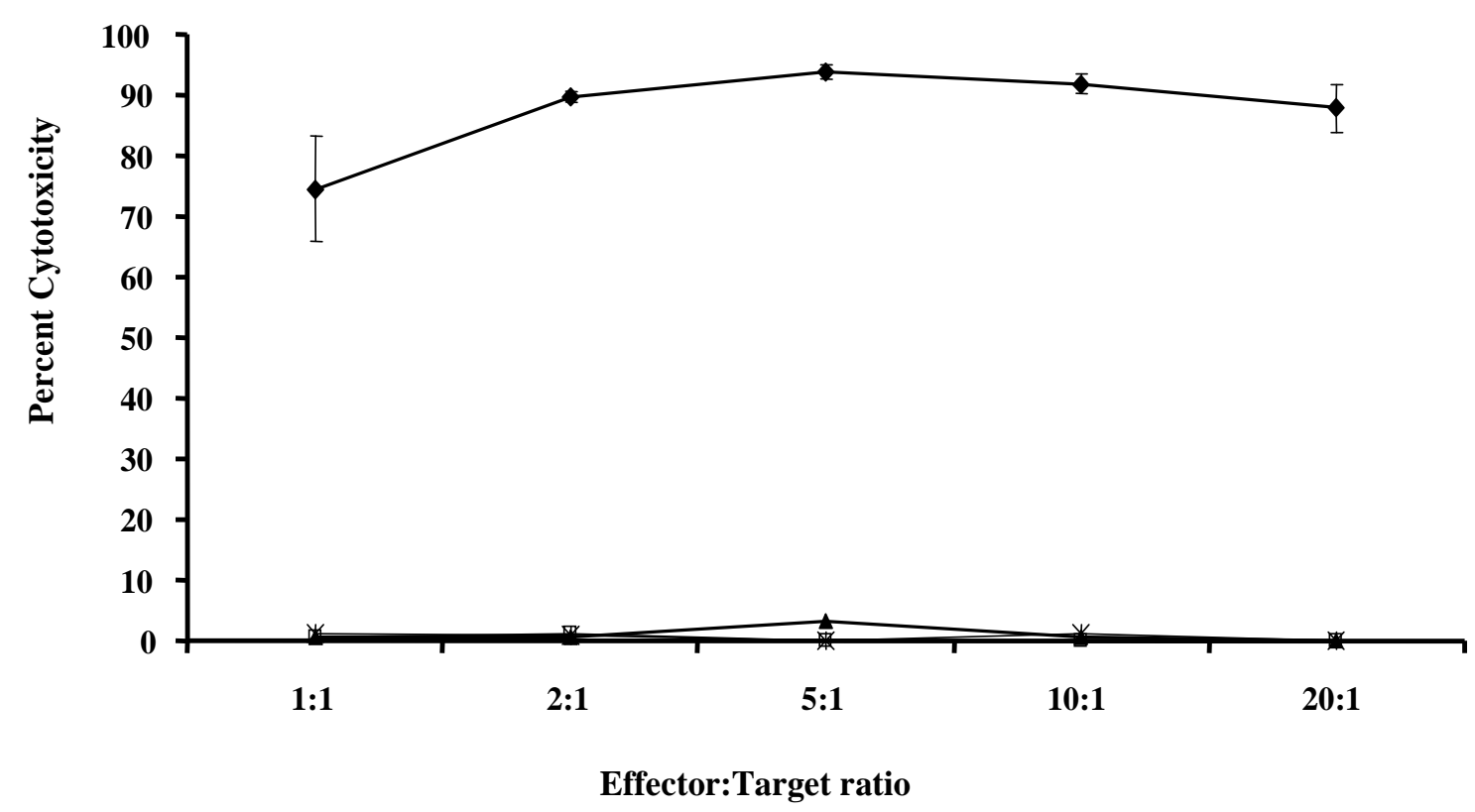

Figure 4-14. Cytolysis of Ob hybridoma by $\mathrm{CDB}^{+}$-MBP-DR2- $\zeta$ RMTC. Ob hMBPspecific or control 6F11 hybridoma were labeled with ${ }^{51} \mathrm{Cr}$ and cocultured with RMTC for four hours. Specific cytolysis was calculated based on chromium release from the dead target cells. $\zeta$-bearing CR-transduced cells were shown to have an exclusive and potent cytolytic activity against MBP-DR2-specific Ob hybridoma, but not control 6F11 cells. 


\subsubsection{Cytolysis of primary antigen-specific T-cells by RMTC}

Based on the ability of RMTC to kill Ob peptide-specific hybridoma, we hypothesized that the same RMTC would also display cytolytic activity against primary antigen-specific T-cells. The results challenged our hypothesis: although $\zeta$-bearing RMTC did indeed kill Ob hybridoma, they failed or poorly killed naïve T-cells from DTG or TTG mice and with very little difference in the cytolytic abilities of the MBPDR2- $\zeta$, MBP-DR2, and MSCV control transduced RMTC (Figure 4-15). A possible explanation would be the fact that, although the residual cells were $\mathrm{hV} \beta 2^{+}$, this does not constitute a direct indication of their antigenic specificity. Indeed, published data using ELISPOT analysis has shown only $0.5 \%$ of T-cells in TCR/DR2 double transgenic mice respond to hMBP84-102 (87). Therefore, we found an alternate approach to avoid stimulation with peptide by stimulating T-cells from these mice with the $\mathrm{DR} 2^{+}$antigenpresenting cell line.

\subsubsection{Cytolysis of primary antigen-specific T-cells cells by RMTC detected by} $\underline{\text { stimulation with DR2 }}{ }^{+}$antigen-presenting cell line

Since primary, naïve antigen-specific cells from DTG animals could not be killed by RMTC probably due to the low number of hMBP84-102-specific cells, we used a different approach to detect residual hMBP-specific T-cells by stimulating target cells with DR2 ${ }^{+}$antigen-presenting cells (EL4-MBP-DR2) as described to selectively expand surviving antigen-specific T-cells. Analysis of residual target cells on day five of stimulation with the hMBP-presenting cell line identified a significantly lower number of peptide-specific cells when they were cocultured with MBP-DR2- $\zeta$ RMTC as opposed to 

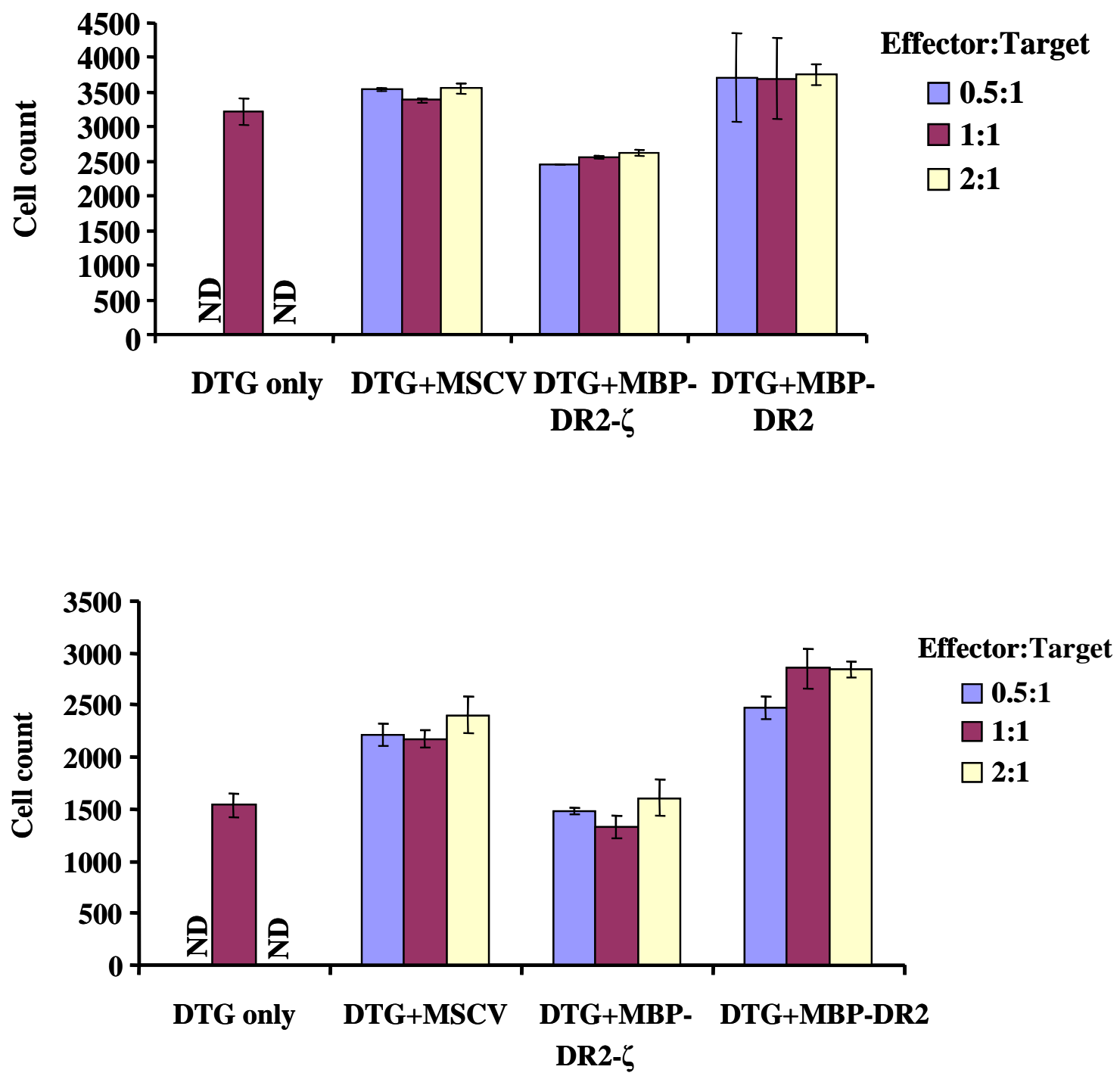

Figure 4-15. $\mathrm{CDB}^{+}$RMTC show limited cytolysis of naïve TCR/DR2 cells from double transgenic (DTG) mice. T-cells from TCR/DR2 DTG mice were cocultured with RMTC in three different ratios overnight. The next day the cells were stained with antihuman V 32 and anti-mouse $\mathrm{CD} 4^{+}$or $\mathrm{CD} 8^{+}$antibody. TruCount beads were added for quantitative assessment of the residual antigen-specific cells. RTMC were not able to efficiently kill primary, hMBP-specific T-cells. Upper panel: overnight cytolysis of CD4 ${ }^{+}$ DTG cells by RMTC; lower panel: overnight cytolysis of CD8 ${ }^{+}$DTG cells by RMTC. 
the number of target cells cocultured with MSCV control or tailless RMTC (Figures 4-16 and 4-17). This leads to two conclusions. The first one is that, by day five after stimulation, the antigen-specific T-cells would undergo clonal expansion, whereas the non-antigen-specific cell would die. The second conclusion is that the significantly lower number of residual CD4 and CD8 antigen-specific cells when naïve target cells were cocultured with MBP-DR2- $\zeta$ CTL is due to killing by CTL as opposed to expansion of target cells were cocultured with MBP-DR2 or MSCV control CTL.

Surprisingly, both $\mathrm{CD} 4^{+}$and $\mathrm{CD} 8^{+}$target cells from TCR/DR2 transgenic animals showed sustained proliferation in response to antigenic stimulation. Human MBP peptide is presented by HLA class II, DR2 gene. The fact that not only CD4 T-cells, but also CD8 T-cells respond to this peptide by proliferation contradicts the dogma of immunology that CD4 T-cells would recognize antigens presented on an MHC class II molecule, whereas CD8 T-cells would recognize antigens complexed with MHC class I (92). This accounts for the coreceptor independence of $\mathrm{CD} 8^{+}$antigen-specific cells from TCR/DR2 transgenic mice and explains their proliferation in response to peptide stimulation.

\subsubsection{Cytolysis of target DTG cell lines by RMTC}

Knowing that peptide-specific cells from DTG mice can be expanded with the $\mathrm{DR}^{+}$antigen-presenting cell line, we were interested in determining whether we could produce hMBP-specific T-cell lines and whether the RMTC could similarly kill these cell lines. $\mathrm{CD} 4^{+}$and $\mathrm{CD} 8^{+} \mathrm{hMBP}$-specific target cell lines were grown as described in Materials and methods (paragraph 4.2.5.2) and cocultured with RMTC. Only the CD4 ${ }^{+}$ MBP-specific cell line was efficiently killed by MBP-DR2- $\zeta$ RMTC, whereas none of the 


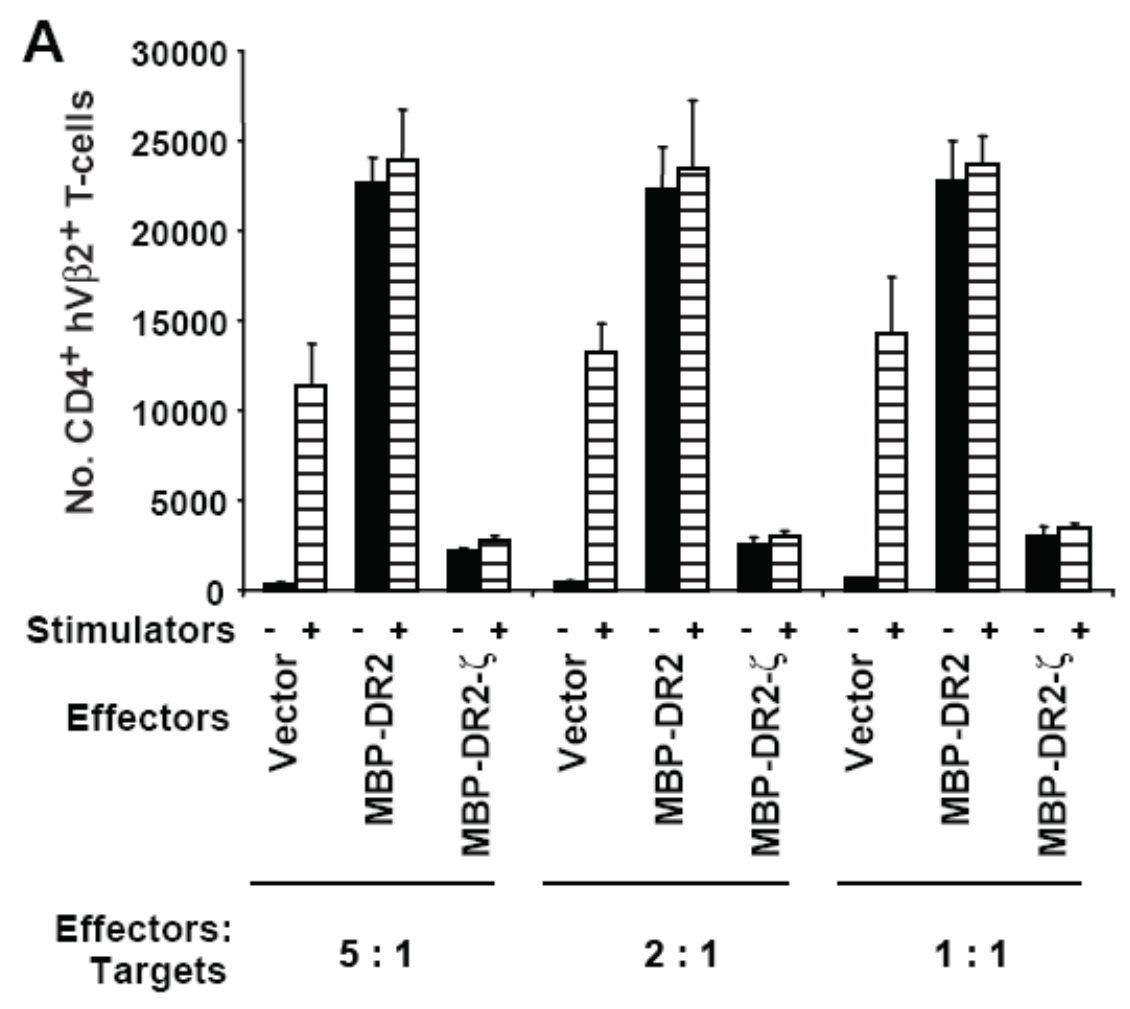

Figure 4-16. Cytolysis of primary CD4 ${ }^{+}$DTG target cells by RMTC measured by stimulation of residual MBP-DR2-specific T-cells. T lymphocytes from DTG mice TCR/DR2 were cocultured with therapeutic cells or MSCV retroviral control in three different ratios in the absence or presence of DR2 ${ }^{+}$antigen-presenting cell line (EL4MBP-DR2) for five days. At the end of the fifth day, the residual cells were stained with $\mathrm{hV} \beta 2$ and mCD4 antibodies and quantitative FACS analysis was performed. We found a considerably lower number of residual $\mathrm{CD}^{+}$peptide-specific cells when these were cocultured with MBP-DR2- $\zeta$ RMTC as opposed to the MSCV control cells or MBP-DR2 RTMC. 


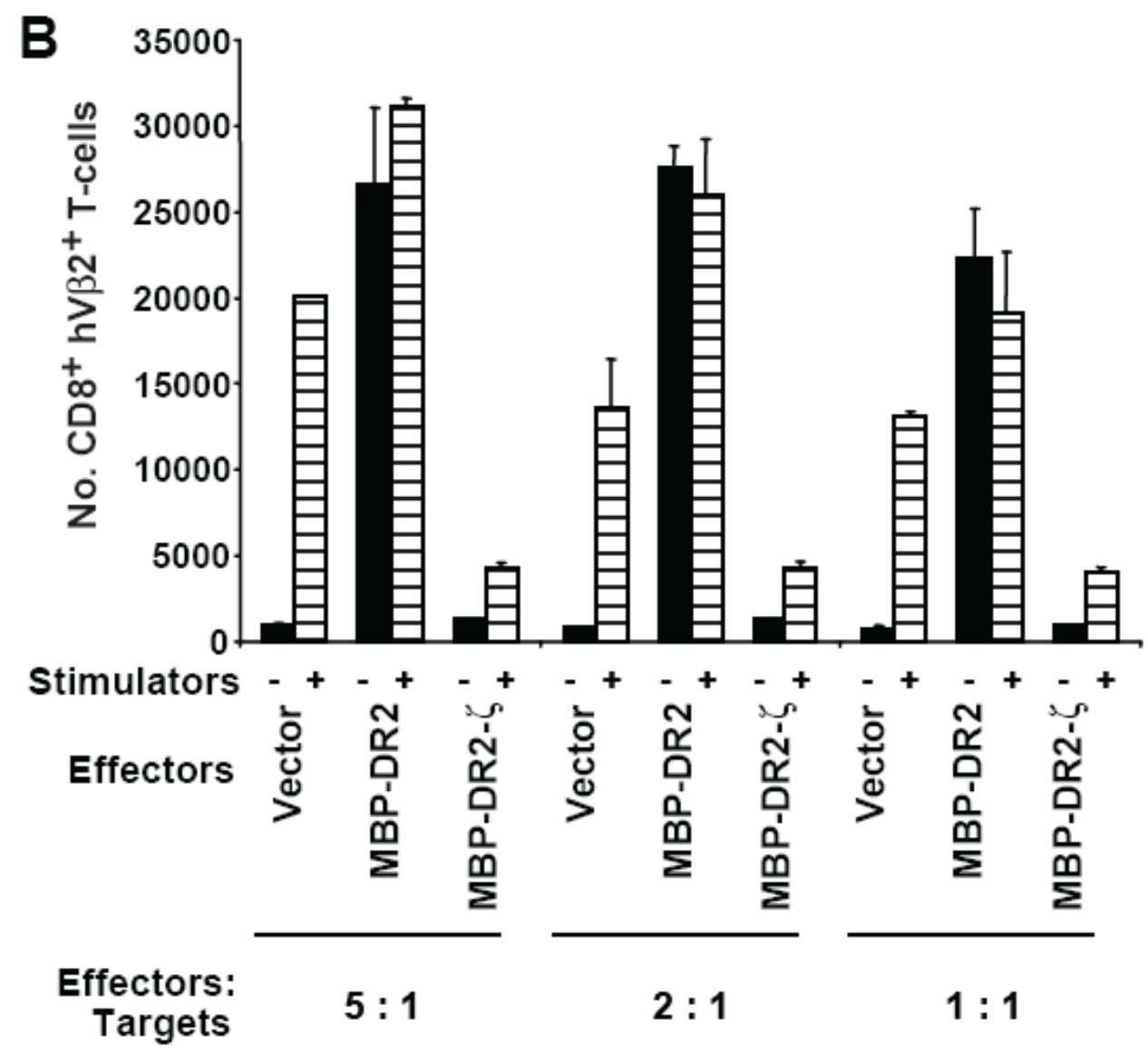

Figure 4-17. Cytolysis of primary $\mathrm{CD8}^{+}$DTG target cells by RMTC measured by stimulation of residual MBP-DR2-specific T-cells. T lymphocytes from DTG mice TCR/DR2 were cocultured with therapeutic cells or MSCV retroviral control in three different ratios in the absence or presence of DR2 ${ }^{+}$antigen-presenting cell line (EL4MBP-DR2) for five days. At the end of the fifth day, the residual cells were stained with $\mathrm{hV} \beta 2$ and mCD4 antibodies and quantitative FACS analysis was performed. We found a considerably lower number of residual $\mathrm{CD}^{+}$peptide-specific cells when these were cocultured with MBP-DR2- $\zeta$ RMTC as opposed to the MSCV control cells or MBP-DR2 RTMC. 
other RTMC (MBP-DR2 and MSCV control) showed this ability. Moreover, both CD4 and CD8 antigen-specific cell lines survived and expanded after six hour-coculture with tailless RMTC or MSCV control cells. Prior data show that RMTC are only able to kill primary $\mathrm{CD}^{+}$antigen-specific T-cells and not stimulated CTL and that activated CD8 ${ }^{+}$ antigen-specific T-cells are resistant to fratricidal lysis (56). Our experience with the antigen-specific T-cell lines from humanized transgenic mice yielded the same results: $\mathrm{CD}^{+}$antigen-specific T-cells can be killed by the tail-bearing RMTC, whereas CD8 ${ }^{+}$ antigen-specific T-cells survived after coculture with MBP-DR2- $\zeta$ RMTC (Figures 4-18 and 4-19).

\subsection{Conclusions}

We developed receptor-modified T-cells that can specifically recognize and target pathologic, antigen-specific $\mathrm{T}$ lymphocytes by transducing chimeric receptors into $4 \mathrm{G} 4 \mathrm{~T}$ cell hybridoma or primary murine T-cells. The MBP-DR2- $\zeta$ CR heterodimer bears an antigen-MHC extracellular domain linked to the functional $\zeta$ signaling moiety from the TCR. The MBP-DR2 CR lacked the cytoplasmic signaling domain. Both constructs were subcloned in MSCV retroviral vector with an IRES site and GFP gene. Their surface expression was proportional to level of expression of the GPF protein. Transduction of 4G4 T cell hybridoma with the $\zeta$-bearing and $\zeta$-deficient retrovirus showed similar surface levels of expression whereas transduction of activated, primary T-cells showed a discrepancy between the two CRs, with a $30 \%$ increase in surface expression of the tailless CR. Upon encounter of cognate TCR on the surface of Ob target hybridoma (specific for hMBP84-102 restricted by HLA-DR2), therapeutic RMTC recognized the 


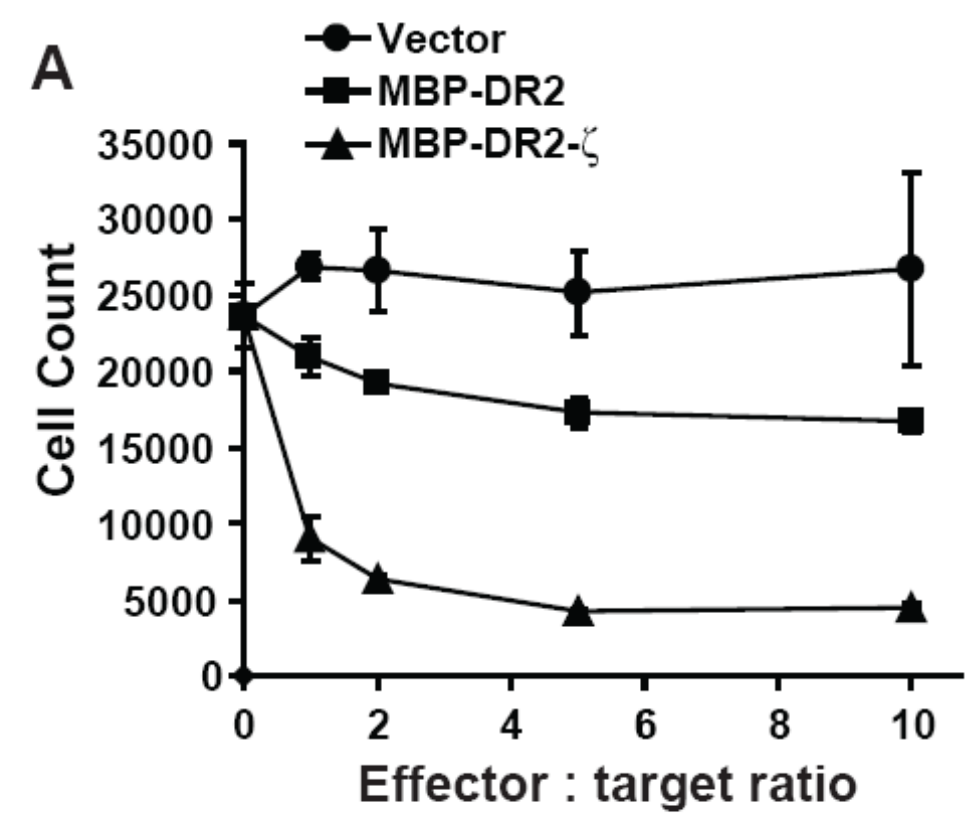

Figure 4-18. Cytolysis of CD4 ${ }^{+}$hMBP-specific cell lines by RMTC. Antigen-specific $\mathrm{CD}^{+}$cell lines were obtained by repeated stimulations of $\mathrm{T}$ lymphocytes from TCR/DR2 DTG mice with EL4-MBP-DR2 cells. Each line was then cocultured with RTMC for six hours and the residual cells were then stained for $\mathrm{hV} \beta 2$ and mCD4 antibodies. MBPDR2- $\zeta$ RMTC were able to efficiently kill the $\mathrm{CD}^{+}{ }^{+}$peptide-specific cell line. 


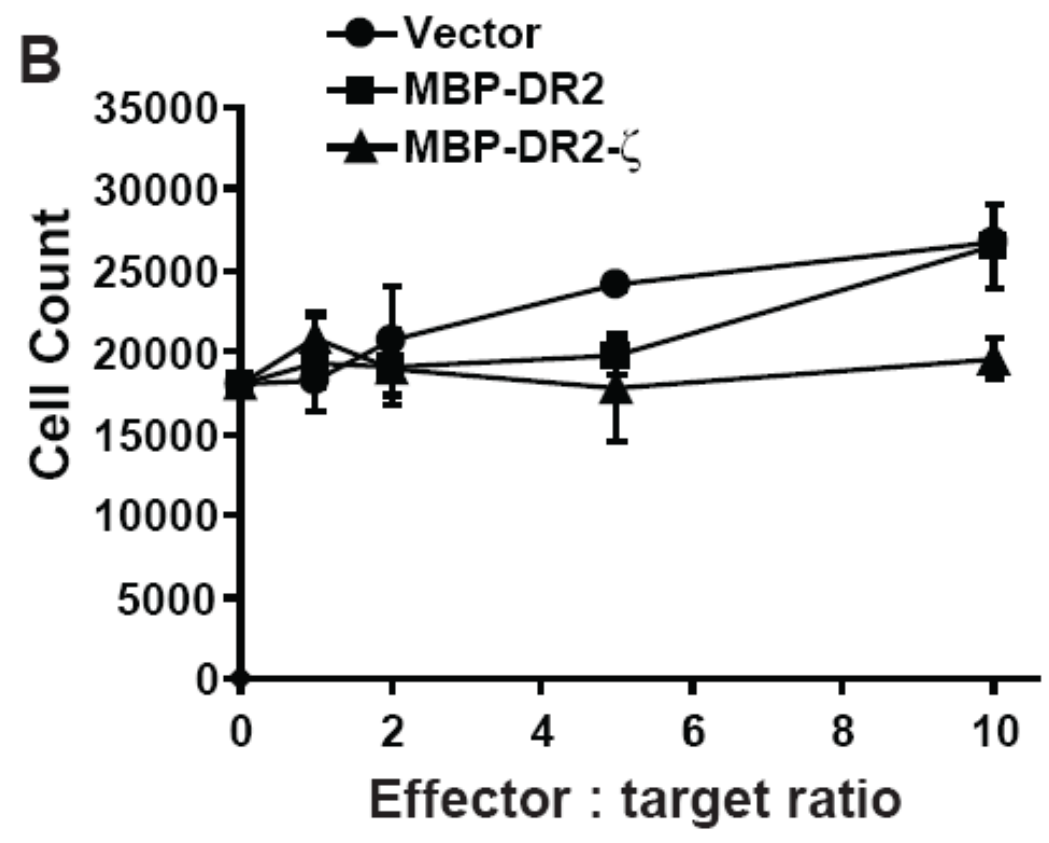

Figure 4-19. Cytolysis of $\mathrm{CD8}^{+}$hMBP-specific cell lines by RMTC. Antigen-specific $\mathrm{CD} 8^{+}$cell lines were obtained by repeated stimulations of $\mathrm{T}$ lymphocytes from DTG mice TCR/DR2 with EL4-MBP-DR2 cells. Each line was then cocultured with RTMC for six hours and the residual cells were then stained for $\mathrm{hV} \beta 2$ and $\mathrm{mCD} 8$ antibodies. Unlike $\mathrm{CD}^{+}$peptide-specific cell lines, the $\mathrm{CD} 8^{+} \mathrm{T}$-cells could not be killed by the MBP-DR2- $\zeta$ RMTC. 
target cells, responded by proliferation, secretion of IL-2 and IFN- $\gamma$, and were able to specifically kill target cells. The in vitro cytolytic ability of CTL RMTC was significant: effector cells killed over $70 \%$ of target cells when cocultured in a 1:1 effector to target ratio and even up to $90 \%$ when five times more effectors were added to the target cells. Cytolysis was only detected in RMTC transduced with the $\zeta$-bearing CRs, but not MSCV control or the $\zeta$-deficient RMTC.

Several attempts to direct RMTC against naïve, antigen-specific T-cells from lymph nodes of TCR/DR2 humanized transgenic mice failed repeatedly in a six hourkilling assay or overnight killing. Analysis of these double transgenic mice revealed that only $0.5 \%$ for their T-cells were peptide-specific cells in an ELISPOT analysis (85). Therefore, the reduced ability of MBP-DR2- $\zeta$ RMTC to specifically kill target cells and the lack of difference between the activity of $\zeta$-bearing and $\zeta$-deficient RMTC and MSCV control was rather due to the very low number of antigen-specific cells that could not be detected and isolated from the non-specific. The latter cells would not be subjected to RMTC lysis, but would interfere in the assay, making identification of specific cytolysis of hMBP-specific T-cell impossible to detect. We adopted an alternate way to stimulate antigen-specific cells using a DR2 ${ }^{+}$antigen-specific cell line (EL4-MBP-DR2) to selectively expand only residual antigen-specific cells. Five days later, we noticed a decreased number of both $\mathrm{CD} 4^{+}$and $\mathrm{CD}^{+}$hMBP-specific T-cells when cocultured with MBP-DR2- $\zeta$ RMTC in the presence of DR2 ${ }^{+}$cell line, but an expansion of these cells when cocultured with MBP-DR2 or MSCV control RMTC. These data show that the $\zeta$ CR-transduced RMTC can also kill naïve antigen-specific T-cells from TCR/DR2 double transgenic mice in the presence of antigen, whereas removal of the signaling domain 
from the CR impeded this ability. Surprisingly, although the humanized transgenic mice have T-cells specific for hMBP presented in the context of DR2, an MHC class II molecule, upon antigenic stimulation, both $\mathrm{CD} 4^{+}$and $\mathrm{CD} 8^{+} \mathrm{T}$-cells from TCR/DR2 mice responded by proliferation. Although fairly rare, this is not an isolate finding and accounts for the coreceptor independence of $\mathrm{CD} 8^{+} \mathrm{TCR} / \mathrm{DR} 2 \mathrm{~T}$-cells.

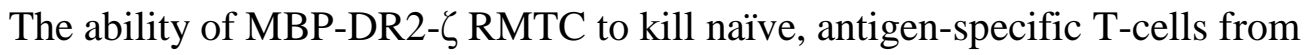
TCr/DR2 mice did not apply in the case of peptide-specific cell lines. Stimulated T lymphocytes behave differently from naïve T lymphocytes. We built a CD4 and a CD8specific cell line by several rounds of antigenic stimulation using DR2 $2^{+}$antigenpresenting cells. Just like naïve CD4 T-cells, peptide-specific $\mathrm{CD}^{+}$cell lines were killed upon in vitro coculture with the tail-bearing RMTC, but not MSCV control or $\zeta$-deficient therapeutic cells. $\mathrm{CD} 8^{+}$hMBP-specific cell line proved resistant to lysis. This fact was demonstrated in the early 1990s and we have also proven that CD8 T-cells acquire resistance to lysis in between day three and seven post stimulation (56). EAE is classically considered a CD4 disease and therefore, the ability of our MBP-DR2- $\zeta$ RMTC to specifically kill both naïve and activated $\mathrm{CD} 4^{+} \mathrm{hMBP}$-specific cell lines are grounds to hypothesize that they might also work in the in vivo mouse model. 


\section{Chapter 5. In vivo activity of RMTC in humanized mice}

\subsection{Introduction}

\subsubsection{Humanized transgenic mice as MS models}

Similar to other autoimmune diseases (rheumatoid arthritis, celiac disease, insulin-dependent diabetes), MS is associated with certain MHC class II genes. The disease is triggered by association of autoantigenic peptides with certain MHC class II; MHC-peptide is recognized by $\mathrm{CD}^{+} \mathrm{T}$-cells that, upon activation, mediate inflammation and destruction of the myelin sheath, ultimately leading to impaired saltatory conduction (93).

In the previous chapter, we described the development of RMTC as therapeutic T lymphocytes bearing humanized chimeric receptors, which can be redirected against antigen-specific T-cells. Upon encounter of target, hMBP-specific T lymphocytes, therapeutic cells will engage the target cells' TCR through their surrogate chimeric receptor, leading to various effector functions, such as proliferation, cytokine secretion, and specific cytolysis of peptide-specific cells.

Once we have shown that RMTC can recognize cognate TCR and exhibit effector functions in response to this recognition, the next step was to test whether this interaction would lead to the same effects in vivo.

In an effort to better understand autoimmune diseases and MS in particular, mice that express disease-relevant MHC II molecules have been developed. These mice were then crossed with mice transgenic for other genes encoding TCRs, human CD4, and 
candidate autoantigens. Since they bear human genes, these mice present similar disease features either spontaneously or after immunization with peptides. Although they are not a perfect representation of the human immunopathology, these animals are, nevertheless, the closest mouse model to study MS-causing mechanisms and symptoms (87).

\subsubsection{Humanized HLA-DR2, TCR, and CD4 mice}

After analyzing autoantigens and MHC molecules associated with MS, studies have shown that one of the immunodominant MBP peptide epitope in $\mathrm{DR} 2^{+}$patients was 84-102. This epitope was identical in humans and mice (94).

Serologic typing of patients serum has identified the common MHC class II molecule as HLA-DRB1*1501 and DRA*0101 that recognized the MBP peptide (86). Based on these data, Fugger and collaborators engineered three humanized transgenic mice. A first mouse bore the human DR2 gene (HLA-DRB1*1501 and DRA*0101) expressed under the control of its native promoters. A second mouse bore a TCR derived from a patient with MS, denoted $\mathrm{Ob}(\mathrm{Ob} .1 \mathrm{~A} 12)$. Because human TCR is difficult to express in mouse cells, the TCR construct comprised human variable domains and mouse constant and regulatory domain. This set-up ensures that the MBP peptide will be able to be recognized and bound by the human MHC class II and, since the constant and signaling domains of the TCR are murine, they could interact with the mouse signaling machinery. These two strains of transgenic mice were crossbred and then also crossed with another transgenic mouse carrying the human CD4 coreceptor. Caveats of this double and triple transgenic mouse model are that only $10-30 \%$ of B-cells were DR2 ${ }^{+}$and only $80 \%$ of T-cells were $\mathrm{V} \beta 2^{+}(87)$. T-cells from these mice showed proliferation and 
IL-2 secretion in response to hMBP84-102 stimulation. Upon immunization with peptide, the mice developed relapsing-remitting or primary progressive EAE similar to MS patients and with similar histopathologic aspect. Single transgenic TCR or HLA-DR2 mice did not develop signs and symptoms of EAE following MBP immunization.

Breeding of the double transgenic TCR/DR2 mice with Rag $2 \%$ mice leads to development of spontaneous EAE due to the complete absence of T and B lymphocytes (including regulatory T-cells) in these knockout mice and repopulation of their immune system with hMBP-specific cells.

\subsection{Materials and methods}

\subsubsection{In vivo cytolysis of $\mathrm{CDH}^{+}$and $\mathrm{CDB}^{+} \mathrm{hMBP}$-specific cell lines by RMTC}

Recombination activating gene 2 knockout mice $(\operatorname{Rag} 2 \%)$ were kept in Helicobacter pylori-free facility of the Animal Research Center at St. Jude Children's Research Hospital. These mice were coinjected with $5 \times 10^{6}$ mixed $\mathrm{CD} 4^{+}$and $\mathrm{CD} 8^{+}$triple transgenic cells from T-cell lines and 20x $10^{6}$ receptor-modified CTLs. CD $4^{+}$and $\mathrm{CD} 8^{+}$ peptide-specific cell lines were obtained by three consecutive stimulations of T-cells from TCR/DR2 transgenic mice with irradiated antigen-presenting cell line (EL4-MBPDR2) every ten days, according to the protocol described in chapter 4.2.5.2. After three days, recipient mice were sacrificed and spleens were processed in a single cell suspension, followed by double staining of splenocytes human V $\beta 2 /$ mouse CD4 and CD8 antibodies and flow cytometry analysis. 


\subsubsection{In vivo cytolysis of naüve antigen-specific T-cells by RMTC}

Irradiated (450 rad) transgenic humanized DR2 mice (strain Fug5) received $10 \times 10^{6}$ freshly isolated T-cells from TCR/DR2 transgenic mice and 30x $10^{6}$ therapeutic RMTC by retroorbital injection. Two irradiated recipient Fug5 mice were used for each group (MSCV control, MBP-DR2- $\zeta$, and MBP-DR2 RMTC).The mice were sacrificed after nine days or the indicated time. Lymph nodes (LN) were collected and processed to obtain a single cell suspension. $10^{5}$ cells/well were stimulated for three days in the presence or absence of antigen ( $2 \times 10^{5}$ EL4-MBP-DR2 cells or EL4 cells, respectively, irradiated at 20,000 rad). 72-hours later, the cells were pulsed with $1 \mu \mathrm{Ci}\left[{ }^{3} \mathrm{H}\right]$ thymidine and harvested on filtermats after 16 hours. Proliferation was measured by liquid scintillation counting of incorporated $\left[{ }^{3} \mathrm{H}\right]$ thymidine. The samples were analyzed in duplicates.

\subsubsection{Prevention of EAE induced by naïve CD4 $4^{+}$-cells from TCR/DR2/hCD4 using RMTC}

Lymph nodes and spleen from ten TCR/DR2/hCD4 transgenic mice were processed in a single cell suspension and stained for PE anti-mouse CD4 microbeads. Cells were then separated by magnetic cell sorting (Miltenyi Biotec Inc., Auburn CA). $13 \times 10^{6}$ pure $\mathrm{CD}^{+} \mathrm{T}$-cells were then transferred into 8 irradiated Fug 5 recipients along with $35 \times 10^{6}$ RMTC. Two days later, the mice were immunized with $300 \mu \mathrm{g}$ hMBP84102 and 400 ng PTx on day 0 and 2. The animals were scored daily for signs of EAE disease and euthanized at score 4 to 5 (complete paralysis or moribund). 


\subsubsection{Prevention of hMBP84-102-induced EAE disease in TCR/DR2 double transgenic}

mice using therapeutic cells

TCR/DR2 transgenic mice were immunized with $300 \mu \mathrm{g}$ hMBP84-102 and at the same time, received the indicated numbers of MBP-DR2- $\zeta$, MBP-DR2 or MSCV control RMTC. 400 ng PTx was injected retroorbitally at the time of peptide injection and on day 2. Mice were followed and scored daily for signs of EAE.

\subsection{Results}

\subsubsection{In vivo cytolysis of $\mathrm{CD}^{+}$and $\mathrm{CDB}^{+} \mathrm{hMBP}$-specific cell lines by $\mathrm{RMTC}$}

This experiment was designed to investigate the in vivo efficacy of therapeutic RMTC in vivo when coinjected with hMBP-specific cell lines into $\mathrm{Rag}^{-}{ }^{-}$mice for three days. Upon interaction with the antigen-specific cell lines, RMTC transduced with chimeric receptor MBP-DR2- $\zeta$ and the MBP-DR2 recognized the specific target cells and exhibited their effector function, namely cytolysis of $\mathrm{CD}^{+}$antigen-specific cells. As expected from previous data, $\mathrm{CD} 8^{+} \mathrm{MBP}$-specific cell line did not undergo cytolysis. Surprisingly, even though MBP-DR2-transduced RMTC failed to show any effector function in vitro due to the absence of the $\zeta$ cytoplasmic signaling tail, in vivo coadoptive transfer of these cells along with MBP-specific cell lines showed almost the same efficacy of killing as the MBP-DR2- $\zeta$ RMTC (Figure 5-1). 


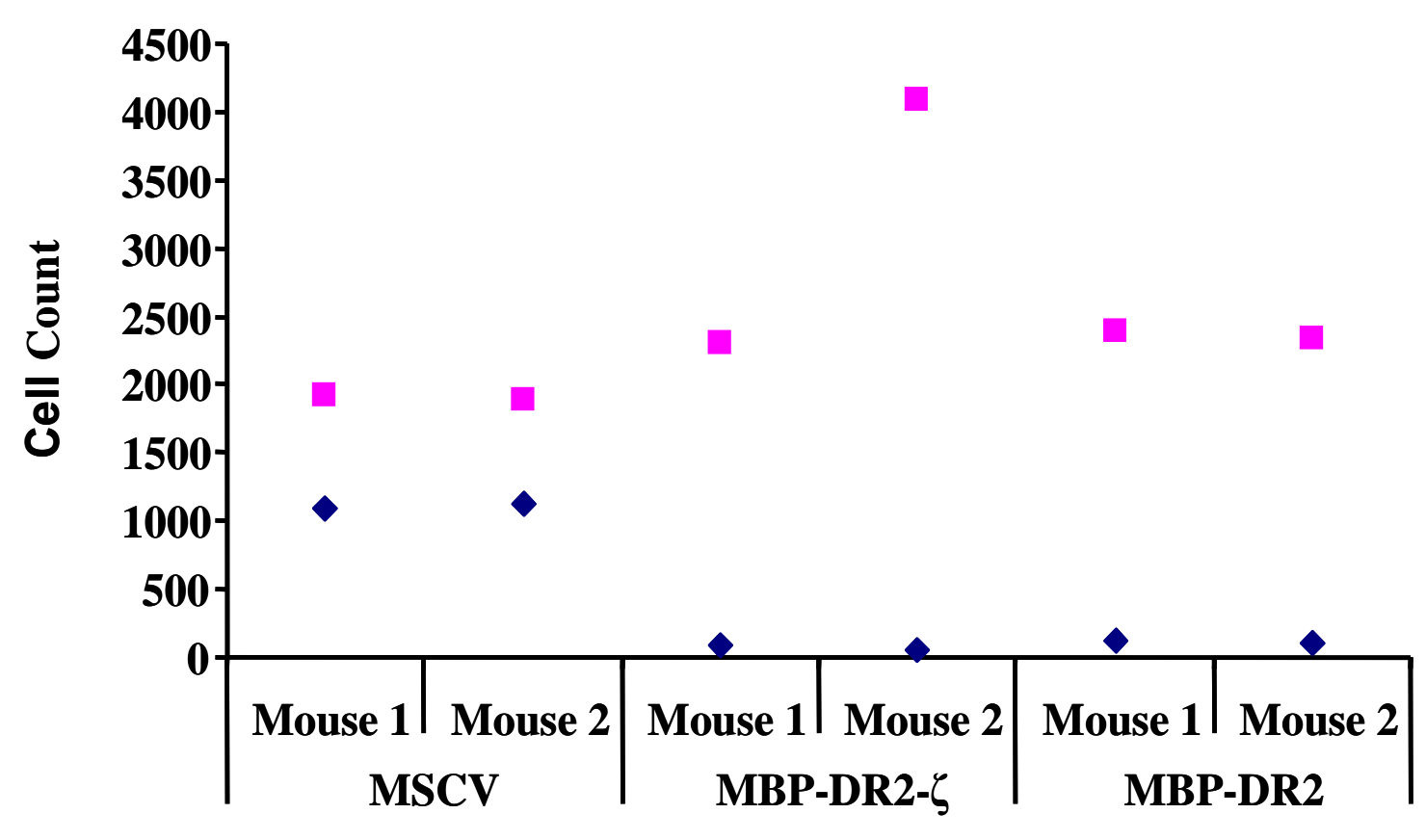

Figure 5-1. In vivo killing of CD4 ${ }^{+} \mathbf{h M B P}$-specific cell line by RMTC. $\mathrm{CD} 4^{+}$and $\mathrm{CD}^{+}{ }^{+} \mathrm{hMBP}$-specific cell lines and RMTC were coinjected into Rag $2 \%$ recipients. Three days later, mice were sacrificed, splenocytes were stained with mouse anti-human V $\beta 2 /$ rat anti-mouse CD4 or CD8 antibodies, and analyzed by FACS. The plot represents the number of cells found in spleens after three days treatment with RMTC. 


\subsubsection{In vivo cytolysis of naüve antigen-specific T-cells by RMTC}

The previous experiment confirmed that $\mathrm{CD} 4^{+}$antigen-specific cell lines could be killed by MBP-DR2- $\zeta$ RMTC, but these effector cells cannot kill CD8 ${ }^{+}$hMBP-specific cell lines. The next step was to find out if whether this also applies to naïve peptidespecific T-cells. We coinjected freshly isolated cells from TCR/DR2 transgenic mice along with RMTC into irradiated recipients, sacrificed them after nine days, and looked for residual peptide-specific cells after stimulation with antigen (irradiated EL4-MBPDR2 cells) or in the absence of antigenic stimulation (irradiated EL4 cells). T-cells from

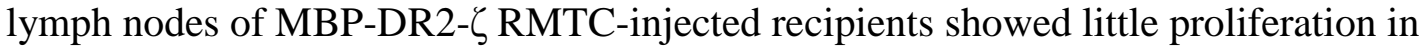
response to antigenic stimulation and decreased number of residual cells compared to the mice that received MBP-DR2 RTMC or MSCV control virus. These results demonstrate that the signaling-competent therapeutic cells effectively eliminate hMBP-specific T-cells in vivo and further demonstrate that signaling through the $\zeta$ cytoplasmic tail is essential for this activity (Figure 5-2).

\subsubsection{Prevention of EAE induced by naïve CD4 ${ }^{+} T$-cells from TCR/DR2/hCD4 using RMTC}

To find out whether EAE induced by adoptive transfer of $\mathrm{CD} 4^{+} \mathrm{T}$-cells from transgenic mice (as described in 5.2.3) could be prevented by MBP-DR2- $\zeta$ RMTC, we followed all three groups of mice daily for signs of disease. The first signs of EAE in the group that received MSCV CTLs became visible after only 7 days and followed a progressive course with very little remission. The mice that received $\zeta$-CR RMTC did not show any signs of disease. This pilot experiment, which requires repetition, shows that 


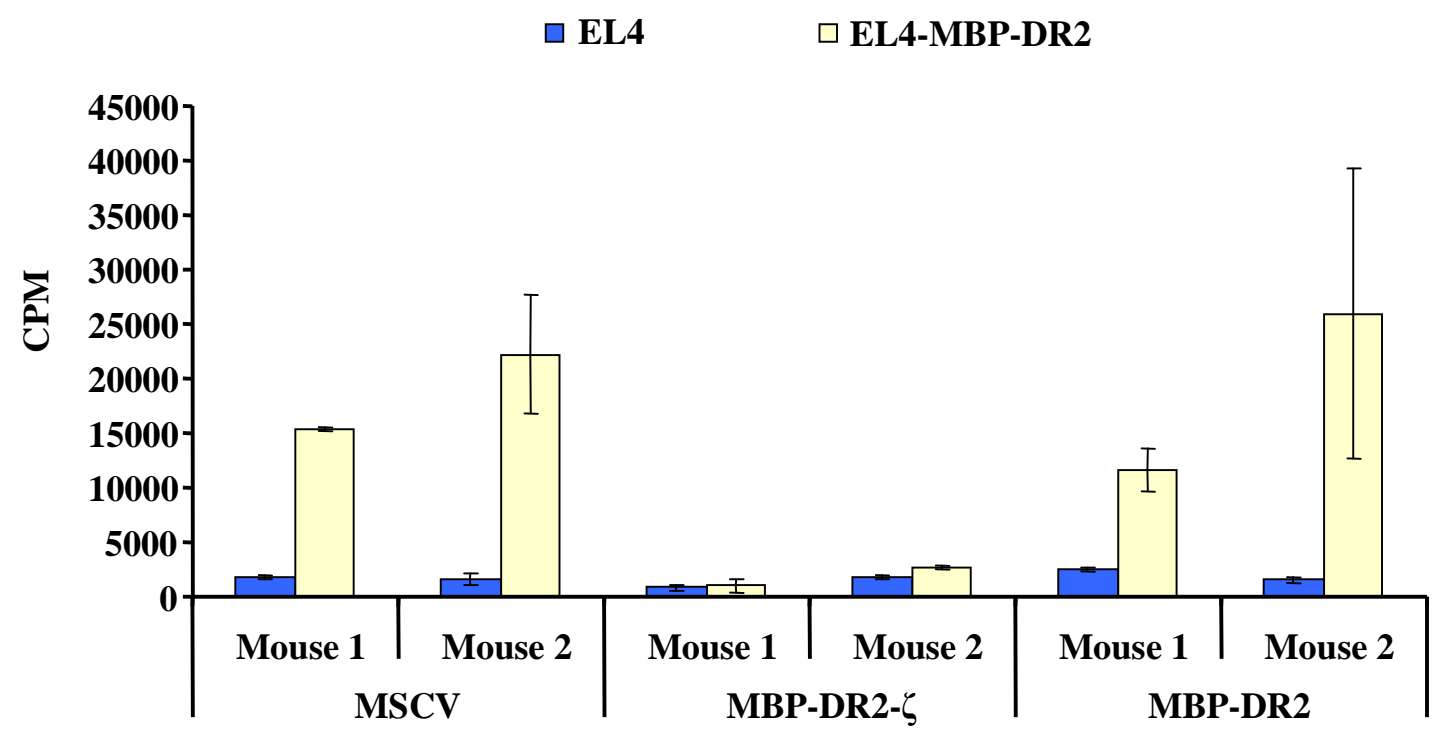

Figure 5-2. Peptide stimulation of T-cells from mice that received RMTC and naïve antigen-specific target cells. Cells from mice that received TCR/DR2 cells and RMTC for nine days were stimulated in the presence/absence of antigen for 72-hours and $\left[{ }^{3} \mathrm{H}\right]$ thymidine was added. 16-hours later, the cells were harvested onto filtermats and proliferation was measured by liquid scintillation counting. hMBP-specific cells from mice that received retroviral vector control and the MBP-DR2 RMTC proliferated in response to antigen as opposed to the ones that received the MBP-DR2- $\zeta$ RMTC. 
upon depletion of $\mathrm{CD} 8^{+} \mathrm{T}$-cells, $\mathrm{CD} 4^{+}$lymphocytes cause and therapeutic RMTC can prevent it. We can conclude that in this particular system EAE is still a CD4-mediated disease and that the MBP-DR2- $\zeta$ RMTC still have therapeutic potential (Figure 5-3 and Table 5-1).

\subsubsection{Prevention of hMBP84-102-induced EAE disease in TCR/DR2 double transgenic mice using therapeutic cells}

This experiment is the first attempt to prevent EAE in the humanized mouse model using humanized chimeric receptor-transduced RMTC. We injected two groups of mice with MSCV control and MBP-DR2- $\zeta$ RTMC and followed them daily for signs of EAE. We first noticed an earlier disease onset in the MSCV control group and also a more aggressive disease course with only mild remission. Contrary to this group, mice that received MBP-DR2- $\zeta$ therapeutic RTMC exhibited a significant delay in the onset of disease as well as a shorter course and complete remission. These in vivo results are confirming our in vitro data and prove the restorative potential of $\zeta$-endowed RMTC (Figure 5-4 and Table 5-2). Even though we did not have any tailless RMTC to inject in the mice (due to a low number of $\zeta$-deficient RMTC that we obtained by in vitro stimulation), this was encouraging data and a further proof that administration of $\zeta$-CRtransduced RMTC might be a valid therapeutic option for EAE treatment in this humanized mouse system. Therefore, we repeated this experiment using $30 \times 10^{6}$ RMTC bearing both chimeric receptors and immunizing the mice two days later. Surprisingly, not only did the MBP-DR2- $\zeta$ RMTC proved beneficial, but so did the tailless therapeutic cells, although not in the same extend as the former ones. This might actually raise 


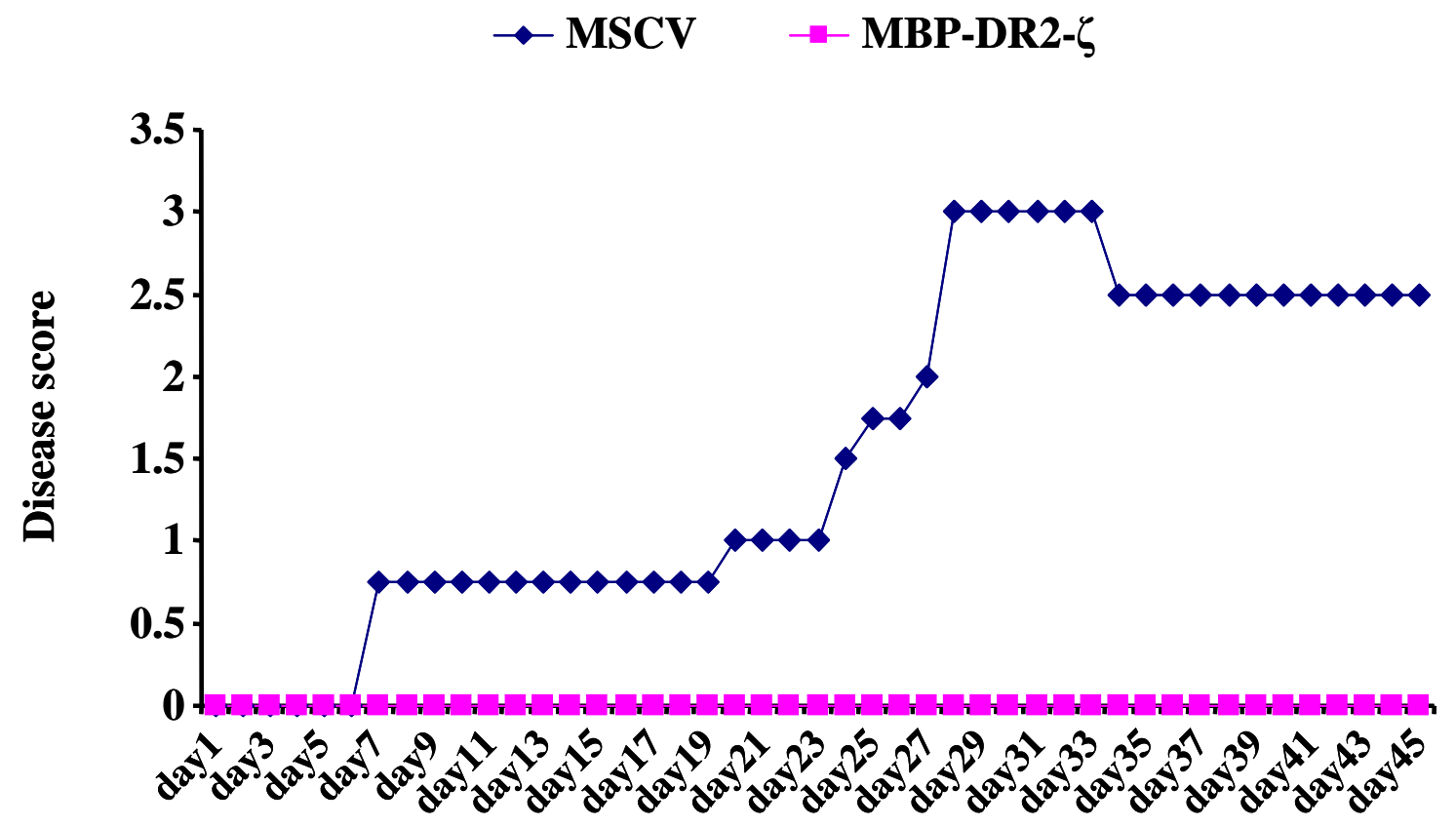

\section{Days after induction}

Figure 5-3. Prevention of $\mathrm{CD4}^{+}$-adoptive transfer EAE with RMTC. Irradiated Fug5 recipients received concomitantly sorted $\mathrm{CD} 4^{+}$target cells from TCR/DR2/hCD4 transgenic mice and therapeutic RMTC followed by immunization with hMBP84-102 peptide and PTx. Recipients of MSCV control cells showed early disease onset and severe course with little remission as opposed to the ones that received therapeutic MBPDR2- $\zeta$ RMTC that were resistant to EAE induction. 
Table 5-1. Clinical manifestations in $\mathrm{CD4}^{+}$adoptive transfer EAE treated with RMTC.

\begin{tabular}{lccc} 
Therapeutic cells & $\begin{array}{c}\text { Disease frequency } \\
\text { (diseased/total) }\end{array}$ & $\begin{array}{c}\text { Mean onset } \\
\text { day } \pm \pm \text { s.d. (range) }\end{array}$ & $\begin{array}{c}\text { Mean maximal } \\
\text { score } \pm \pm \text { s.d. }\end{array}$ \\
\hline MSCV & $4 / 4$ & $9.5 \pm 7$ & $3 \pm 2.3$ \\
MBP-DR2- $\zeta$ & $0 / 0$ & $0 \pm 0$ & $0 \pm 0$ \\
\hline
\end{tabular}



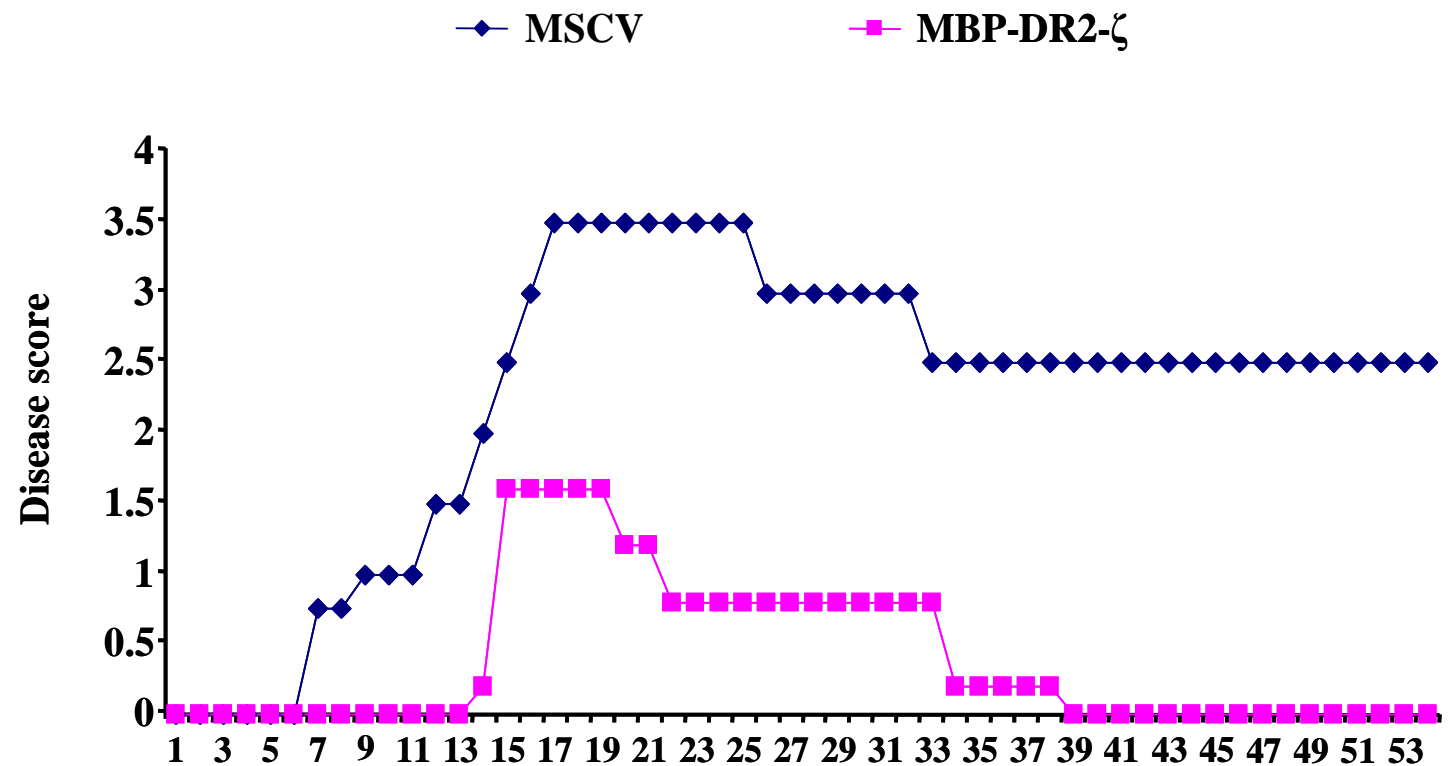

Days after induction

Figure 5-4. Prevention of EAE with $\zeta$-bearing RMTC. TCR/DR2 mice received MBPDR2- $\zeta$ RMTC or MSCV control cells and were immunized with peptide and PTx. Control group showed early onset and severe disease course, whereas treated group had a milder form of disease followed by complete remission. 
Table 5-2. Clinical manifestations in direct induction of EAE with hMBP and treatment with MSCV vector control or $\zeta$-bearing RMTC.

\begin{tabular}{cccc} 
Therapeutic cells & Disease frequency & Mean onset & Mean maximal \\
& (diseased/total) & day $\mathrm{x} \pm$ s.d. (range) & score \pm \pm s.d. \\
\hline MSCV & $4 / 4$ & $8.75 \pm 3.5$ & $3.5 \pm 1.7$ \\
MBP-DR2- $\zeta$ & $4 / 5$ & $11.8 \pm 6.6$ & $1.6 \pm 1.1$ \\
\hline
\end{tabular}


question about the absolute requirement for the cytoplasmic signaling tail in the chimeric receptor for the in vivo treatment of EAE (Figure 5-5 and Table 5-3).

\subsection{Conclusions}

The results obtained from in vitro testing of RMTC encouraged us to move forward to the in vivo system. We coinjected therapeutic RMTC along with either peptide-specific cell lines or naïve antigen-specific cells obtained from the transgenic mice into recipient $\mathrm{Rag}^{-} /$or irradiated DR2 (Fug5) mice. We sacrificed the mice at various intervals, starting from day 3 to 9, and estimated the number of residual CD4 and CD8 antigen-specific T-cells. According to our in vitro data, therapeutic RMTC bearing the $\zeta$-signaling tail should be able to specifically recognize and kill naive antigen-specific T-cells. When we sacrificed recipient mice nine days after coinjection of MBP-DR2- $\zeta$ and naïve target cells, we found a decreased number of $\mathrm{hV} \beta 2^{+} \mathrm{CD} 4^{+} / \mathrm{CD} 8^{+} \mathrm{T}$-cells. Since we have already established that very few of the target cells provided by TCR/DR2 mice are antigen-specific, we stimulated cells from lymph nodes and spleen of recipient mice with antigen as previously described. T-cells from irradiated recipients that received MBP-DR2 and MSCV-control RMTC along with naïve antigen-specific cells showed good proliferation upon peptide stimulation unlike mice that received MBP-DR2- $\zeta$ RMTC. These data resemble the ones we obtained from in vitro experiments and prove that recognition through surrogate chimeric receptor also takes place in the mouse system. Surprisingly, when $\mathrm{Rag}^{\top} /$ recipients received both therapeutic cells and hMBPspecific cell lines, both $\zeta$-bearing and $\zeta$-deficient RMTC were effective in eliminating the $\mathrm{CD}^{+}$peptide-specific cell line. We did not pursue this observation, but the fact that 


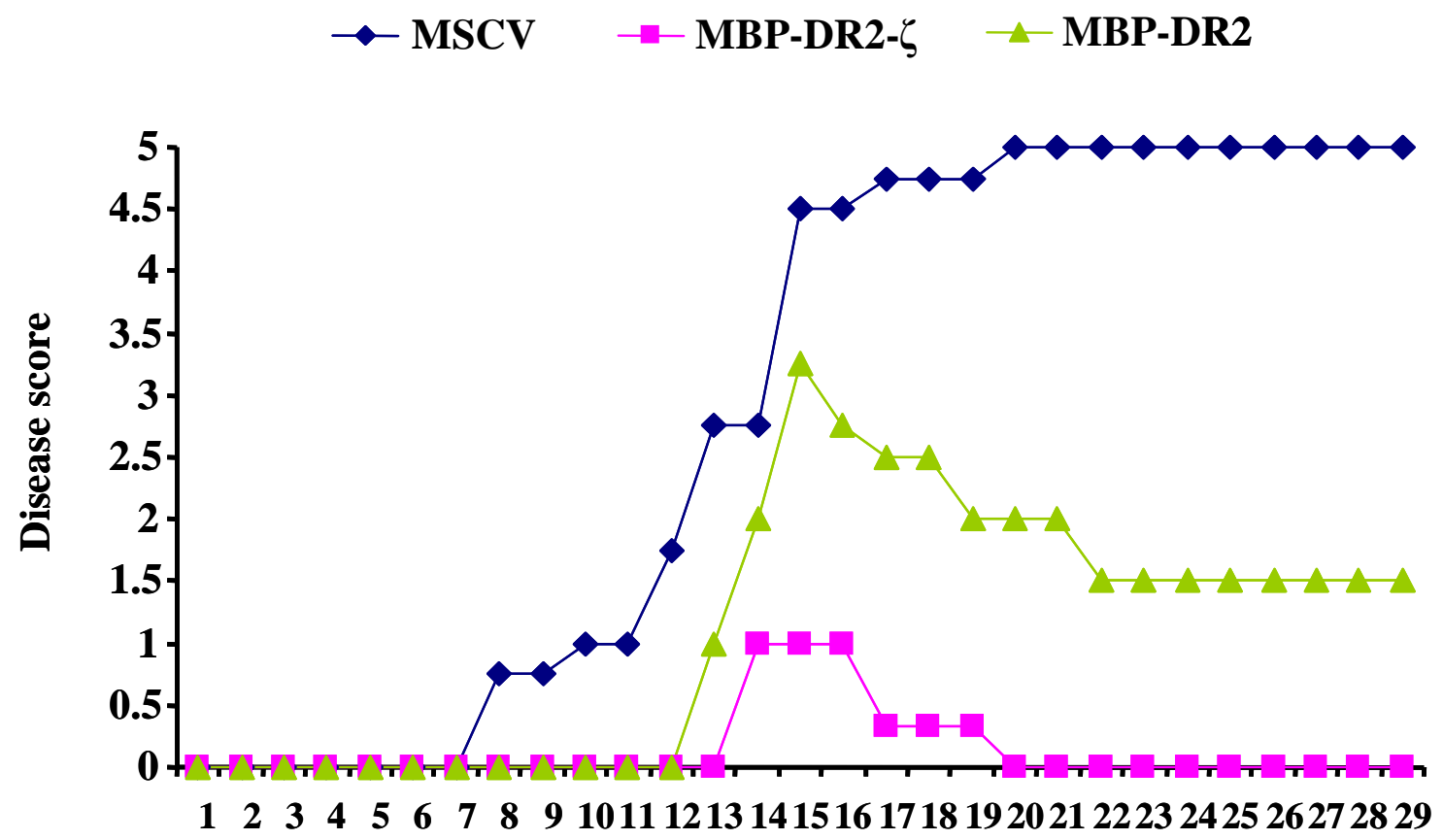

Days after induction

Figure 5-5. Prevention of EAE with $\zeta$-bearing or tailless RMTC. Three groups of TCR/DR2 transgenicmice received MBP-DR2- $\zeta$, MBP-DR2, and MSCV control RTMC and 48 hours later peptide and PTx. Control group showed early onset and severe disease course, whereas $\zeta$-competent therapeutic cells showed a later onset and milder disease followed by complete remission. $\zeta$-deficient therapeutic cells showed an intermediate effect, but still managed to induce remission although not in the same extent as MBP-

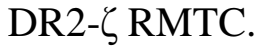


Table 5-3. Clinical manifestations in direct induction of EAE with hMBP and treatment with MSCV control, $\zeta$-bearing, or tailless RMTC.

\begin{tabular}{lccc} 
Therapeutic cells & $\begin{array}{c}\text { Disease frequency } \\
(\text { diseased/total) }\end{array}$ & Mean onset & Mean maximal \\
& $4 / 4$ & $8.5 \pm 1$ & score $\mathrm{d} \pm$ s.d. \\
\hline MSCV & $3 / 3$ & $14 \pm 0$ & $5 \pm 0$ \\
MBP-DR2- $\zeta$ & $4 / 4$ & $13 \pm 0$ & $1 \pm 0$ \\
MBP-DR2 & & & $3 \pm 1.4$ \\
\hline
\end{tabular}


stimulated cells are more prone to Fas-induced apoptosis might provide an explanation for this finding. Just like the in vitro data, RMTC with or without signaling tail were unable to kill the $\mathrm{CD}^{+}$peptide-specific cell line.

We proceeded to test the ability of chimeric receptor-modified T-cells to prevent EAE disease either by direct injection of these cells into recipient, TCR/DR2 mice, followed by immunization with hMBP84-102, or by adoptive transfer of therapeutic cells along with target cells into DR2 ${ }^{+}$mice. Adoptive transfer of both RMTC and freshly isolated, naïve cells from TCR/DR2 mice resulted in worse disease scores and earlier onset of paresis and paralysis in mice that received MSCV or tailless RMTC compared to those that received MBP-DR2- $\zeta$ T-cells. This does not necessarily mean that therapeutic, $\zeta$-bearing RMTC are less potent than they have shown to be in vitro, but rather insufficient number of cells compared to the number of antigen-specific, naïve target cells. Our next step was to sort CD4 and CD8 T-cells from TCR/DR2 transgenic mice and only adoptively transfer $\mathrm{CD} 4^{+}$T-cells and RMTC into irradiated DR2 ${ }^{+}$humanized transgenic mice, followed by immunization with peptide. There was no development of EAE in mice that received MBP-DR2- $\zeta$ RMTC, as opposed to MSCV-control cells. Although we did not have MBP-DR2 RMTC available for this particular experiment, the fact that $\zeta$-CR-transduced effector cells could efficiently eradicate $\mathrm{CD} 4^{+}$target cells is proof that these therapeutic cells are potent not only in vitro but also in vivo in EAE prevention. Finally, upon direct transfer of therapeutic cells into TCR/DR2 mice and induction of EAE disease by peptide immunization, mice that received $\zeta$-bearing RMTC showed later onset of disease with milder course. Quite unexpectedly and unlike our in vitro data, $\zeta$-deficient RMTC recipients have also shown a milder EAE course and later 
onset compared to MSCV control mice, though their potency was lower than the one exhibited by the $\zeta$-bearing therapeutic cells.

All these data lead us to believe that surrogate $\zeta$-chimeric receptor-transduced RMTC as just as effective in killing antigen-specific T-cells from TCR/DR2 mice in vivo as they were in vitro, thus preventing EAE disease. A question that rises naturally is whether these therapeutic cells would also work after the first signs of EAE disease in forestalling the fatal outcome and improving the clinical signs and symptoms, and this may be a topic for future studies. 


\section{Chapter 6. Discussion}

Multiple sclerosis is the most common autoimmune disease involving the white matter of the CNS. It is reported to affect 300,000 Americans with 200 people diagnosed weekly and 2.5 million people worldwide (2). The neurologic signs and symptoms of MS are varied and can also present in other diseases. Double vision, paralysis, tremor, numbness, and gait difficulties are caused by demyelination resulting in impaired nerve conduction; axons and cell bodies are spared early in the disease. The initial disease target is the white matter of the brain and spinal cord, but after a while the gray matter also becomes involved due to secondary degeneration resulting from the loss of the white matter. Axons will eventually be destroyed and replaced by gliosis that gives the sclerotic aspect on MRI.

There are currently several therapies approved for MS. Regardless of the approach, treatment should target both the symptoms and the pathologic cascade.

Gene therapy is the newest approach in the treatment of MS. It has emerged as a direct result of increased knowledge concerning pathologic mechanisms in autoimmune diseases and aims to more specifically target pathologic T-cells than current therapies. Anti-inflammatory cytokine genes can be delivered into the CNS by viral vectors. More recently, our laboratory has developed receptor-modified T-cells bearing chimeric receptors that specifically target autoreactive T-cells. A similar approach has already been used in infectious diseases and cancer.

Chimeric receptors are hybrid molecules that encompass a recognition domain the variable region involved in antigen recognition from $\mathrm{MHC}$ or $\mathrm{Ig}-$ and a signaling 
domain - the intracytoplasmic signaling moieties of the TCR. This new therapeutic approach emerged in the 1980s-1990s when it was found that T-cells endowed with antibody-like abilities and no MHC restriction can be redirected against infected and neoplastic cells. The necessity of having a signaling domain resides in the fact that recognition of antigen by the variable region of the chimeric receptor is not sufficient to activate T-cells. Similar to the cascade of events triggered in a regular T lymphocyte, Tcells bearing chimeric receptors undergo phosphorylation of ITAMs on the receptor's signaling moiety and turn on the transcription of nuclear factors responsible for activation of these cells, thus inducing effector function. Engagement of the TCR constitutes the first signal necessary for activation of $\mathrm{T}$ lymphocytes but for a full activation a second signal is mandatory. This can be through either of several molecules including the CD28 molecule that stimulates the expansion of $\mathrm{CD}^{+}$cells and CD137 (or 4-1BB) that prevents activation-induced cell death of activated T-cells and stimulates the expansion of $\mathrm{CD}^{+}$cells.

The choice of the cytoplasmic signaling domain has long been a critical topic in the design of chimeric receptors. Increasing the number of ITAMs did not necessarily enhance signaling of the CR (95). In an attempt to test the effect of different signaling domains on the potency of signal transduction, several intracytoplasmic domains have been used in a CR engineered to bear the recognition domain of MHC class $\mathrm{I}\left(\mathrm{K}^{\mathrm{b}}\right)$ molecule. The combinations of CD28-zeta, zeta-lck, CD4-zeta, and CD28-zeta-lck signaling domains increased receptor function measured by IL-2 secretion after stimulation with the anti- $\mathrm{K}^{\mathrm{b}}$ specific antibody. Although CRs bearing the intracytoplasmic CD28 moiety showed very high 
efficacy of signal transduction, their surface expression level decreased compared to CRs that only had $\zeta$ signaling tail. We examined the sequence of CD28 and found a previously unrecognized dileucine motif. Dileucine motifs are normally present very close to the membrane in the cytoplasmic tail of transmembrane proteins and are recognized by coat proteins involved in lysosomal targeting. Proteins bearing this amino acid sequence are internalized and transported to the endosome and eventually to the lysosome, decreasing their surface expression. We hypothesized that a leucine to glycine $(\mathrm{L} \rightarrow \mathrm{G})$ mutation in the dileucine motif could potentially not only improve the surface expression of the construct, but also the duration and intensity of the signal transduced. Indeed, when stimulated with the CR-specific antibody, anti- $\mathrm{K}^{\mathrm{b}}$, there was an enhanced production of IL-2 from $\mathrm{CD} 8^{+}$T-cells transduced with the $\mathrm{K}^{\mathrm{b}}-\mathrm{CD} 28(\mathrm{~L}-\mathrm{G})-\zeta$ compared with cells transduced with $\mathrm{K}^{\mathrm{b}}-\zeta \mathrm{CR}$. In addition, there was an increased proliferative response to the antibody and increased cytotoxic activity against $\mathrm{K}^{\mathrm{b}}$-restricted target cells. These data show that a mutation in the dileucine motif of the CD28 intracytoplasmic moiety increases the intensity of the signal transduction events.

The primary rationale for transducing chimeric receptors into $\mathrm{T}$ lymphocytes in MS is the production of therapeutic, receptor-modified T-cells able to tolerize or even kill autoantigen-specific target cells. Autoreactive T-cells are normally stimulated upon encounter of an antigenic peptide bound to MHC on the surface of APCs. Since CRs can bear both an MHC class II recognition domain (extracellular and transmembrane parts) that has the peptide linked to the surface and the intracytoplasmic signal transduction domain, upon transduction into T lymphocytes the cells will acquire both APC and TCR abilities (peptide presentation and $\mathrm{T}$ cell effector function). The encounter between 
effector and target cells bearing the chimeric receptor and a normal TCR respectively, leads to stimulation of both cell populations. Stimulation of RMTC is a critical feature of this system because it leads to the fulfillment of their effector functions: proliferation, cytokine secretion, and cytolysis of target. In a mouse system, therapeutic T-cells transgenic for heterodimeric CRs bearing the recognition domain of MHC class II IA ${ }^{\mathrm{s}} \beta$ chain linked to MBP89-101 and a $\zeta$ signaling tail paired with IA $^{\mathrm{s}} \alpha \underline{\text { chain-}}-\zeta$, were able to express the CR on the surface and were stimulated by MBP89-101-specific 6F11 target hybridoma. They secreted cytokines, proliferated, and managed to kill autoreactive $\mathrm{CD} 4^{+}$ T-cells and were not killed by them. These data show that the two cell types interact via CR-cognate TCR recognition and effector function occurs following this recognition.

The key goal in using RMTC is the prevention and/or treatment of EAE disease. SJL/J mice immunized with MBP89-101 peptide were treated with therapeutic cells or PBS control at the time of disease induction. The treated animals were completely protected from EAE and suppression of disease was antigen-specific. Although protection was more efficient when the animals received therapeutic cells concomitantly with antigenic peptide, administration of cells even after the onset of disease symptoms was still effective, albeit slightly less so and with a delayed kinetic compared with treatment at the time of immunization. The explanation for this may reside in the status of the autoreactive T-cells. In the first scenario, the cells did not experience a previous encounter with antigen and are, therefore, naïve, whereas in the second situation, upon immunization with peptide, target antigen-specific T-cells had already been stimulated, expanded, and mediated some damage to the CNS.

The mechanism by which RTMCs exercise their therapeutic function is inhibition 
of antigen-specific $\mathrm{T}$ cell response of target cells which may occur through the killing of the target cells. In addition to this, pathologic cells are skewed from Th1 to Th2 type and secrete anti-inflammatory cytokines, such as IL-4, instead of IFN- $\gamma$.

In the work described here, we proposed extending our studies in murine EAE by developing a new model for the therapy of MS using humanized chimeric receptors. We designed two constructs based on the same principle that has already been described for CRs. The first construct, MBP-DR2- $\zeta$, had a recognition domain composed of an immunodominant MBP epitope (84-102) linked to the $\beta$ chain of a HLA class II molecule (DR2) and a signaling domain composed of the $\zeta$ cytoplasmic signaling tail. The $\alpha$ chain of DR2 was also linked to $\zeta$. The DR2 HLA molecule is highly associated with MS disease, and T-cells specific for the MBP84-102 epitope can be detected in DR2 patients with MS. We then engineered a second CR, MBP-DR2 that lacked the signaling tails to identify the role of signal transduction for the in vitro and in vivo efficacy of this particular CR. The constructs were each tricistronic, with the $\beta$ and $\alpha$ chains separated by a 17-mer 2A sequence from T. asigna and an IRES linked to GFP. Both constructs were subcloned into MSCV retroviral vector. CRs were transduced in either 4G4 TCRhybridoma or primary murine T lymphocytes from $\mathrm{C} 57 \mathrm{Bl} / 6$ mice, sorted for GFP expression and expanded in complete Bruff medium and IL-2.

The tail-bearing and tailless CRs showed good surface expression on flow cytometry in both 4G4 and primary T-cells when stained with anti-CR specific antibody mouse anti-human DR2. Transduced hybridoma showed similar levels of expression of CR; transduced T-cells showed better expression of the tailless CR compared to the $\zeta$ tailbearing one ( $71.7 \%$ versus $40.4 \%)$. 


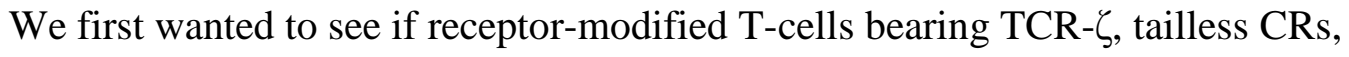
or MSCV retroviral vector control could be stimulated by plate-bound DR2-specific antibody. Only MBP-DR2- $\zeta$ responded to stimulation by secreting IL-2.

We next looked for the ability of CR-transduced T-cells to recognize the cognate TCR on the surface of target cells. The latter were either Ob1A12 hybridoma specific for hMBP84-102/DR2 T-cells or T cell lines from the double or triple transgenic mice, TCR/DR2 and TCR/DR2/hCD4, respectively, with similar specificity. These humanized mice have develop a disease that strongly resembles human MS model; they carry one of the most frequent immunodominant epitope of myelin basic protein, $84-102$, presented by one of the most frequently used class II MHC in MS patients, DRB1*1501. Even though very few of the T-cells in the transgenic mice were MBP-specific in Tg mice bred on a $\mathrm{Rag}^{+/+}$background, on a Rag// background all of the T-cells will express exclusively the Tg receptor and these mice develop spontaneous EAE. We further demonstrated that both CD4 cells and CD8 cells from the double Tg mice responded to MBP peptide restricted to the DR2 (DRB1*1501) HLA molecule. Thus the T cell response in these mice is not coreceptor dependent. As controls we used other targets, specifically 6F11 hybridoma reactive against MBP89-101/IA ${ }^{\mathrm{s}}$ and T-cells from non-Tg mice.

Upon recognition of cognate TCR on the surface of Ob1A12 specific hybridoma but not $6 \mathrm{~F} 11$ controls, RMTC bearing the $\zeta$ signaling tail were stimulated and secreted IL-2 and IFN- $\gamma$. Also, due to the interaction between the two cells, RMTC and target Ob1A12 cells, RMTC proliferated and killed the target cells. The cytolytic effect was specific and very effective as even at a 1:1 ratio effector to target ratio, the RMTC managed to eliminate over $70 \%$ of the targets. It is noteworthy that only the cells bearing 
the cytoplasmic signaling tail were capable of both proliferation and killing upon recognition of cognate TCRs.

Our attempt to demonstrate similar results in cytolysis assays using primary murine antigen-specific cells from lymph nodes or spleens of DTG (TCR/DR2) mice did not yield the same results. In vitro overnight killing assays in which RMTC targeted Tcells from transgenic mice showed very little difference between control and experimental cultures. This seemed be due to the fact that although $80 \%$ of T-cells in the humanized transgenic mice were $\mathrm{hV} \beta 2^{+}$, only $\sim 0.5 \%$ of them are hMBP84-102-specific in ELISPOT assays. Therefore a preponderance of non-antigen-specific cells that cannot be recognized and killed by RMTC were present therefore preventing detection of any target cell lysis.

We approached this problem in two ways. First, we developed a functional assay to overcome it in which we stimulated T-cells with antigen in vitro for five days. The MBP-specific cells survive, get activated, and proliferate in response to peptide, whereas the non-specific cells die. After 5 days we could demonstrate that virtually all surviving T-cells are antigen specific. We treated cultures at the time of antigen-stimulation either with control MSCV-vector transduced RMTC, MBP-DR2 RMTC or MBP-DR2- $\zeta$ RMTC. After 5 days we used quantitative flow cytometry to determine the number of surviving T-cells. This demonstrated that the MBP-specific cells were killed by $\zeta$ bearing RMTC, whereas the target cells cocultured with MSCV control or tailless RMTC proliferated due to peptide stimulation. Surprisingly, these results were true not only for $\mathrm{CD}^{+}$antigen-specific cells, but also for the $\mathrm{CD} 8^{+} \mathrm{MBP}-$ specific cells.

As a second approach, we established T cell lines using T-cells from DTG 
(TCR/DR2) or TTG (TCR/DR2/hCD4) mice stimulated repeatedly with exogenous antigen (irradiated EL4-MBP-DR2 cells) and used them to test the cytolytic ability of RMTC. Results showed that, unlike the previous experiment where therapeutic cells bearing the signaling tail were capable of killing naïve, CD4 and CD8 antigen-specific Tcells, the RMTC could not achieve the same outcome with $\mathrm{CD} 8^{+} \mathrm{hMBP}$-specific cell line. $\mathrm{CD}^{+} \mathrm{T}$-cells develop resistance to lysis between days 3 and 7 after activation and this might explain the lack of potency of the RMTC in killing the $\mathrm{CD}^{+}$cell line (57). It may also impair the therapeutic usefulness of RTMCs in EAE models, such as potentially the TCR/DR2 model of ours, where class II restricted CD8 T-cells may have a pathologic role.

We went on to test our RMTC in vivo. These studies highlighted the in vitro results and provided evidence for the therapeutic applicability of humanized RMTC. Adoptive transfer of freshly isolated lymphocytes from humanized transgenic mice TCR/DR2 along with therapeutic cells into irradiated humanized DR2 recipients showed decreased proliferation upon antigenic stimulation in mice treated with the $\zeta$ - bearing RMTC as opposed to a vigorous proliferative response generated in mice that received MSCV control or tailless RMTC.

Adoptive transfer of antigen-specific cell lines and therapeutic cells into $\operatorname{Rag}^{\top}{ }^{\top}$ mice followed by treatment with RMTC yielded somewhat different results. As we saw in vitro, the number of $\mathrm{CD}^{+}$peptide-specific cell line cells was not diminished by either $\zeta$ - or tailless RMTC when compared with the control treatment. Therefore these cells seemed to be resistant to lysis in vivo, much as they were in vitro. Results with the CD4 cell line were more surprising. Not unexpectedly, the number of residual CD4 peptide- 
specific cells was decreased in mice injected with MBP-DR2- $\zeta$ RMTC. However, mice injected with MBP-DR2 RMTC showed a similar and nearly complete loss of these cells. This in vivo effect of the tailless CR-transduced cells could not be predicted based on any of the in vitro experiments. One potential explanation for the difference is that stimulation by the MBP-DR2 RMTC in vivo induces the MBP-reactive cell line but not naive T-cells to undergo activation induced cell death. However, at the current time experimental evidence to support this does not exist.

The ultimate goal of this project is to prevent or treat EAE in a humanized mouse model. We adopted two approaches. The first one was to adoptively transfer cells from triple transgenic TCR/DR2/hCD4 mice along with therapeutic cells followed by immunization with hMBP and PTx. The mice were observed and scored for EAE disease for 50 days. Recipients of the zeta-bearing therapeutic cells showed later onset of disease symptoms and milder course compared to the ones receiving MSCV control or zetadeficient RMTC. We have also tried to induce EAE by adoptive transfer of $\mathrm{CD}^{+}$cells from TTG mice and therapeutic cells followed by peptide immunization. MBP-DR2- $\zeta$ CTL completely blocked development of EAE disease, whereas mice that received MSCV CTL developed EAE within a week from injection. Since EAE is classically considered a CD4 ${ }^{+}$Th1-induced disease, the efficient elimination of these cells by the zeta-bearing therapeutic cells is an indication of their potency in blocking the disease. We also noticed that the mice that received MSCV control cells along with $\mathrm{CD}^{+}$target cells developed less severe signs of EAE than the mice that received unsorted target cells which might account for the role of $\mathrm{CD}^{+} \mathrm{MBP}-$ specific cells as "inducers" of EAE in this particular mouse model. Due to the lack of humanized MHC class II mice (Fug5) we 
could not repeat these experiments, but this is a task we intend to pursue in the future. We also intend to test the ability of therapeutic cells to block disease induced by adoptive transfer of $\mathrm{CD}^{+}$cells from TCR/DR2/hCD4 transgenic mice.

The second approach for in vivo prevention of EAE disease involved the use of TCR/DR2 double transgenic mice as recipients of therapeutic cells. These mice received active peptide immunization and PTx two days after the transfer of RTMCs. Although these experiments need to be repeated, they yielded very encouraging results: mice that received zeta-bearing RMTC showed a delayed onset of disease, a milder course, and remission compared to the mice that received control CTL. The tailless chimeric receptor-transduced T-cells seemed to be efficient as well, though not in the same extent as the ones bearing the zeta signaling tail. Two more experiments validating these data are ongoing.

These studies are important for several reasons. The use of RMTC expressing humanized CR in humanized transgenic mice brings us one step closer to their use in treating multiple sclerosis in humans. Experimental therapies in humans are limited by both technical and ethical considerations whereas mice have similarities but are simpler to use as disease models. Conversely, although genetically similar, mice are not humans, and some therapeutic approaches that proved beneficial for them have not worked in humans or could even be harmful. HLA-transgenic mice are particularly useful in modeling autoimmune diseases associated with specific HLA alleles, such as MS. Although it is rather difficult to identify exactly the genes responsible for MS, due to the strong linkage disequilibrium, HLA class II has a clear contribution in predisposing to the development of disease (85). HLA is crucial as it governs T cell selection in the thymus 
and antigen presentation in periphery. Upon binding of a TCR to a peptide antigen in the groove of MHC II, a cascade of signal transduction events ensues.

CD4 costimulation may be required as a TCR interacts with MHC. The requirement in the humanized mouse model we have studied is less clear. The class II MHC used in this model is entirely human in sequence. Data from literature are extremely controversial on whether murine CD4 can bind human class II MHC. Some studies demonstrate that an entirely human MHC class II mouse cannot interact with mouse CD4 $(96,97)$. Therefore, in some humanized systems, a mixed, chimeric human/mouse class II molecule was engineered so that murine CD4 coreceptor could bind the $\beta 2$ domain (also murine) of the transgenic HLA II (98). Another set of data seemingly contradicted this requirement. Altman et al. engineered humanized HLA-DR1 transgenic mice and crossbred them with human CD4 transgenic mice (Hu-CD4) and tested the response of $\mathrm{Hu}-\mathrm{CD} 4$ positive versus $\mathrm{Hu}-\mathrm{CD} 4$ negative T-cells to influenza haemagglutinin (HA). T-cells proliferated strongly to this peptide regardless the CD4 status (99). This suggests the possibility of interaction between human HLA class II and mouse CD4; however this was not formally proven. In our experience, using antigenspecific T-cells from double transgenic or triple transgenic mice (including or lacking $\mathrm{Hu}-\mathrm{CD} 4)$ did not make a difference in the experimental results.

Another issue regarding our system is the proliferative response of $\mathrm{CD}^{+}$antigenspecific T-cells to peptide stimulation. EAE is classically considered a CD4 Th1 disease (100) and until recently the research was predominantly focused on this T cell phenotype. One study that used MOG35-55 as the immunodominant epitope showed equal proliferative potential of both CD4 and CD8 cells to this antigen and EAE induction by 
adoptive transfer of CD8 antigen-specific cells (28). On the same note, the inflammatory infiltrate in the brain of the patients with MS incriminated both CD4 and CD8 T cell types along with B cells, and depletion of CD4 cells in MS patients only minimally decreased relapse rates (101). Invasion of the CNS after intravenous delivery of MBPactivated, cytotoxic CD8 cells result in very severe ascending flaccid paralysis with high mortality (102). The aggressive and somewhat different manifestation of disease is a critical aspect for therapy as CD8-induced disease can be dampened by anti-IFN- $\gamma$ antibodies (unlike the CD4-induced disease), while anti-TNF- $\alpha$ does not show any effect on the disease course. It is also noteworthy that perivascular inflammation leading to further demyelination in MS is composed mainly of $\mathrm{CD} 8^{+} \mathrm{T}$-cells as these cells recognize endogenous antigens processed via the MHC class I pathway.

$\mathrm{CD}^{+} \mathrm{T}$-cells have been incriminated as culprits for MS. As a logical consequence, the humanized mice that we used for this project might actually be closer to the human disease than EAE mediated exclusively by CD4 T-cells, as the disease in this model might be caused by both CD4 and CD8 autoreactive T-cells. Since HLA-DR2 mice bear human MHC class II, but not class I, a CD4 response to hMBP-stimulation is to be expected. All our experiments showed a proliferative ability of both $\mathrm{CD} 4^{+}$and $\mathrm{CD}^{+}{ }^{+} \mathrm{MBP}$-specific cells. Under the circumstances, we hypothesized that there are MHC class II-restricted $\mathrm{CD} 8^{+} \mathrm{T}$-cells able to respond to peptide stimulation.

If $\mathrm{CD} 8^{+} \mathrm{T}$-cells play a prominent pathologic role in disease, they might become problematic for our therapeutic approach since we transduced the chimeric receptors into $\mathrm{CD} 8^{+} \mathrm{T}$-cells that can only target and kill $\mathrm{CD}^{+}{ }^{+}$antigen-specific cell lines, but not $\mathrm{CD} 8^{+}$ antigen-specific cell lines. On the other hand, our RMTC are able to kill both cell types as 
long as they are still naïve. Chimeric receptor-transduced CTL can still efficiently kill long-term activated antigen-specific $\mathrm{CD} 4^{+} \mathrm{T}$-cells, whereas activated $\mathrm{CD} 8^{+} \mathrm{MBP}$-specific cells become resistant to lysis. Under these circumstances, we attempted to prevent rather than treat disease by injecting therapeutic cells before EAE induction. This way we ensured very early cytolysis of the majority CD4 and CD8 naïve, antigen-specific cells. Indeed, when treated with zeta-bearing CR-transduced RMTC the mice developed EAE disease later than controls, had less severe disease, and developed a complete remission. A sensible explanation would be that the RMTC inhibited both CD4 and CD8 naïve, antigen-specific cells.

Both in vitro and in vivo experiments using RMTC as a therapeutic tool did not show complete cytolysis or abrogate proliferation of target cells from TCR/DR2 mice. A rational question would be why residual MBP-specific cells could not get activated, proliferate, and induce disease. There are several possible explanations for this issue. One would be that the therapeutic cells are very potent in killing autoreactive T-cells and therefore, the low number of residual target cells would not be able to cause disease. This finding is true for both $\mathrm{CD}^{+}$and $\mathrm{CD}^{+}$population. A second reason refers to the relationship between $\mathrm{CD}^{+}$and $\mathrm{CD}^{+} \mathrm{T}$-cells in the development of EAE disease. If CD4 T-cells are effectively eliminated, CD8 T-cells would not be able to survive without the signals delivered by CD4 T helper cells. Therefore, although the chimeric receptor was transduced into $\mathrm{CD}^{+} \mathrm{CTL}$ that can only target and kill CD4 hMBP-specific cells, their disappearance would also impede survival of $\mathrm{CD} 8^{+} \mathrm{hMBP}-$ specific cells.

The in vivo efficacy of the $\zeta$-deficient RMTC is still an open question. Removal of the cytoplasmic signaling tail impeded signal transduction and recognition of target $\mathrm{T}$ - 
cells by MBP-DR2-CTL. MBP-DR2 therapeutic cells cannot kill hMBP-specific cells. Likewise, they cannot inhibit proliferation of antigen-specific cells after peptide stimulation. The only option left that might explain is the conversion of autoreactive, Th1 cells intoTh2 ones and secretion of anti-inflammatory cytokines (IL-4, 5, 13). This is one of the hypotheses that remain to be proven in the future.

A major limitation in the therapy of EAE is the phenomenon of epitope spread. The disease in primarily induced by a particular antigenic peptide that activates T-cells but due to local inflammation and damage, the T-cell response will extend to other epitopes within the same antigenic protein or different proteins (103). Naïve T-cells can penetrate the inflamed CNS without the need of peripheral activation (104). Dendritic cells in the CNS will then activate these naïve T-cells, thus initiating epitope spread (105). This phenomenon occurs after the peak of acute disease, around day 16 after immunization with a specific peptide and it is inhibited by treatment with IFN- $\beta$ (106). HLA-DR15/MBP85-99 humanized transgenic mice were shown to undergo epitope spread to other HLA-DR15-restricted MBP epitopes. We do not know if this would also happen in our humanized system. Previous reports from our lab using RMTC specific for MBP89-101 reactive T-cells after epitope spread showed symptomatic improvement of EAE-induced mice. In addition lymphocytes from the mice showed decreased proliferation not only to MBP89-101, but also to two PLP epitopes, 139-151 and 178191, that are important in epitope spread (58). Although these constitute encouraging data, we can not assume that humanized chimeric receptor-transduced therapeutic cells would also block epitope spread in our humanized system and this is an important question to be addressed in the future. 
The ultimate goal of this entire project using T-cells transduced with chimeric receptors for the therapy of MS in a humanized mouse model is to eventually use this approach in human clinical trials. Several adjustments should be made for the chimeric receptors to meet the safety requirements of such trials. The GFP gene is suitable for experiments in mice but it induces an immune response in humans (107). Therefore, this gene should be removed as the RMTC can be easily detected and quantified by staining with the DR2 antibody. Moreover, since there is very little data about the side-effects of RMTC presenting an autoantigen, a suicide mechanism would be beneficial so that their prompt elimination becomes readily possible. One way to do this is insertion of herpes simplex virus thymidine kinase gene (hsvTK) into the transduced construct. This converts the dideoxynucleoside prodrug gancyclovir into its phosphorylated form, thus terminating the DNA chain and leading to cell death (108).

In this project we only tested the ability of $\mathrm{CD}^{+} \mathrm{T}$-cells transduced with $\mathrm{CRs}$ to prevent or treat EAE in the humanized system. There are several other variations to this theme. One of them is transduction of CRs into Th2 lymphocytes or regulatory $\mathrm{CD} 4^{+} \mathrm{CD} 25^{+} \mathrm{T}$-cells. These therapeutic cells will not directly kill autoreactive, peptidespecific cells, but rather act through different mechanisms, such as anti-inflammatory cytokines. In this manner they may be more potent than the CD8+ RMTC cells in settings where significant epitope spreading has already taken place. The field is still wide open for the use of CRs and other, more "classical", non antigen-specific approach for the fight for the cure of this disease. 


\section{LIST OF REFERENCES}

1. Nguyen, P., I. Moisini, and T. L. Geiger. 2003. Identification of a murine CD28 dileucine motif that suppresses single-chain chimeric T-cell receptor expression and function. Blood 102:4320-4325.

2. Pugliatti, M., S. Sotgiu, and G. Rosati. 2002. The worldwide prevalence of multiple sclerosis. Clinical Neurology and Neurosurgery 104:182-191.

3. Barcellos, L. F., S. Sawcer, P. P. Ramsay, S. E. Baranzini, G. Thomson, F. Briggs, B. C. A. Cree, A. B. Begovich, P. Villoslada, X. Montalban, A. Uccelli, G. Savettieri, R. R. Lincoln, C. DeLoa, J. L. Haines, M. A. Pericak-Vance, A. Compston, S. L. Hauser, and J. R. Oksenberg. 2006. Heterogeneity at the HLADRB1 locus and risk for multiple sclerosis. Human Molecular Genetics 15:28132824.

4. A. D. Sadovnick, H. Armstrong, G. P. A. Rice, D. Bulman, L. Hashimoto, D. W. Paty, S. A. Hashimoto, S. Warren, W. Hader. T. J. Murray. 1993. A populationbased study of multiple sclerosis in twins: Update. Annals of Neurology 33:281285.

5. Detels, R., B. R. Visscher, R. W. Haile, R. M. Malmgren, J. P. Dudley, and A. H. Coulson. 1978. Multiple sclerosis and age at migration. American Journal of Epidemiology 108:386-393.

6. Confavreux, C., S. Vukusic, T. Moreau, and P. Adeleine. 2000. Relapses and progression of disability in multiple sclerosis. New England Journal of Medicine 343:1430-1438.

7. Calabresi, P. A. 2004. Diagnosis and management of multiple sclerosis. American Family Physician 70:1935-1944.

8. Smith, K. J., and W. I. McDonald. 1999. The pathophysiology of multiple sclerosis: the mechanisms underlying the production of symptoms and the natural history of the disease. Philosophical Transactions of the Royal Society of London 354:1649-1673.

9. Olson, J. K., J. L. Croxford, M. A. Calenoff, M. C. Dal Canto, and S. D. Miller. 2001. A virus-induced molecular mimicry model of multiple sclerosis. Journal of Clinical Investigation 108:311-318.

10. Neuropathology:http://www.neuropathologyweb.org/chapter6/chapter6aMS.html, accessed on January, 2007.

11. Dhib-Jalbut, S., D. L. Arnold, D. W. Cleveland, M. Fisher, R. M. Friedlander, M. M. Mouradian, S. Przedborski, B. D. Trapp, T. Wyss-Coray, and V. Wee Yong. 2006. Neurodegeneration and neuroprotection in multiple sclerosis and other neurodegenerative diseases. Journal of Neuroimmunology 176:198-215.

12. McRae, B. L., C. L. Vanderlugt, M. C. Dal Canto, and S. D. Miller. 1995. Functional evidence for epitope spreading in the relapsing pathology of experimental autoimmune encephalomyelitis. Journal of Experimental Medicine $182: 75-85$. 
13. Herman, A., J. W. Kappler, P. Marrack, and A. M. Pullen. 1991. Superantigens: mechanism of T-cell stimulation and role in immune responses. Annual Review of Immunology 9:745-772.

14. Langrish, C. L., Y. Chen, W. M. Blumenschein, J. Mattson, B. Basham, J. D. Sedgwick, T. McClanahan, R. A. Kastelein, and D. J. Cua. 2005. IL-23 drives a pathogenic T-cell population that induces autoimmune inflammation. Journal of Experimental Medicine. 201:233-240.

15. Gocke, A. R., P. D. Cravens, L. H. Ben, R. Z. Hussain, S. C. Northrop, M. K. Racke, and A. E. Lovett-Racke. 2007. T-bet regulates the fate of Th1 and Th17 lymphocytes in autoimmunity. Journal of Immunology 178:1341-1348.

16. Chen, Y., C. L. Langrish, B. McKenzie, B. Joyce-Shaikh, J. S. Stumhofer, T. McClanahan, W. Blumenschein, T. Churakovsa, J. Low, L. Presta, C. A. Hunter, R. A. Kastelein, and D. J. Cua. 2006. Anti-IL-23 therapy inhibits multiple inflammatory pathways and ameliorates autoimmune encephalomyelitis. Journal of Clinical Investigation. 116:1317-1326.

17. Neumann, H., I. M. Medana, J. Bauer, and H. Lassmann. 2002. Cytotoxic T lymphocytes in autoimmune and degenerative CNS diseases. Trends in Neurosciences 25:313-319.

18. Vass, K., and H. Lassmann. 1990. Intrathecal application of interferon gamma. Progressive appearance of MHC antigens within the rat nervous system. American Journal of Pathology 137:789-800.

19. Rivers, T. M., D. H. Sprunt, and G. P. Berry. 1933. Observation on attempts to produce acute dissmeinated encephalomyelitis in monkeys. Journal of Experimental Medicine 58:39-53.

20. Kabat, E. A., A. Wolf, and A. E. Bezer. 1947. The rapid production of acute disseminated encephalomyelitis in Rhesus monkeys by injection of heterologous and homologous brain tissue with adjuvants. Journal of Experimental Medicine 85:117-130.

21. Paterson, P. Y. 1960. Transfer of allergic encephalomyelitis in rats by means of lymph node cells. Journal of Experimental Medicine 111:119-136.

22. Zamvil, S. S., and L. Steinman. 1990. The T lymphocyte in experimental allergic encephalomyelitis. Annual Review of Immunology 8:579-621.

23. Bergman, R. K., J. J. Munoz, and J. L. Portis. 1978. Vascular permeability changes in the central nervous system of rats with hyperacute experimental allergic encephalomyelitis induced with the aid of a substance from Bordetella pertussis. Infection \& Immunity. 21:627-637.

24. Oliveira-dos-Santos, A. J., A. Ho, Y. Tada, J. J. Lafaille, S. Tonegawa, T. W. Mak, and J. M. Penninger. 1999. CD28 costimulation is crucial for the development of spontaneous autoimmune encephalomyelitis. Journal of Immunology 162:4490-4495.

25. Gold, R., C. Linington, and H. Lassmann. 2006. Understanding pathogenesis and therapy of multiple sclerosis via animal models: 70 years of merits and culprits in experimental autoimmune encephalomyelitis research. Brain 129:1953-1971.

26. Schluesener, H. J., R. A. Sobel, C. Linington, and H. L. Weiner. 1987. A monoclonal antibody against a myelin oligodendrocyte glycoprotein induces 
relapses and demyelination in central nervous system autoimmune disease. Journal of Immunology 139:4016-4021.

27. Erik Wallström, M. Khademi, M. Anderosson, R. Weissert, C. Linington, T. Olsson. 1998. Increased reactivity to myelin oligodendrocyte glycoprotein peptides and epitope mapping in HLA DR2 $(15)^{+}$multiple sclerosis. European Journal of Immunology 28:3329-3335.

28. Ben-Nun, A., H. Wekerle, and I. R. Cohen. 1981. The rapid isolation of clonable antigen-specific $\mathrm{T}$ lymphocyte lines capable of mediating autoimmune encephalomyelitis. European Journal of Immunology 11:195-199.

29. Sun, D., J. N. Whitaker, Z. Huang, D. Liu, C. Coleclough, H. Wekerle, and C. S. Raine. 2001. Myelin antigen-specific $\mathrm{CD} 8^{+} \mathrm{T}$-Cells are encephalitogenic and produce severe disease in C57BL/6 mice. Journal of Immunology 166:7579-7587.

30. Tompkins, S. M., J. Padilla, M. C. Dal Canto, J. P. Ting, L. Van Kaer, and S. D. Miller. 2002. De novo central nervous system processing of myelin antigen is required for the initiation of experimental autoimmune encephalomyelitis. Journal of Immunology 168:4173-4183.

31. Goverman, J., A. Woods, L. Larson, L. P. Weiner, L. Hood, and D. M. Zaller. 1993. Transgenic mice that express a myelin basic protein-specific $\mathrm{T}$ cell receptor develop spontaneous autoimmunity. Cell 72:551-560.

32. Brabb, T., A. W. Goldrath, P. von Dassow, A. Paez, H. D. Liggitt, and J. Goverman. 1997. Triggers of autoimmune disease in a murine TCR transgenic model for multiple sclerosis. Journal of Immunology 159:497-507.

33. Krishnamoorthy, G., H. Lassmann, H. Wekerle, and A. Holz. 2006. Spontaneous opticospinal encephalomyelitis in a double-transgenic mouse model of autoimmune T cell/B cell cooperation. Journal of Clinical Investigation 116:2385-2392.

34. Milligan, N. M., R. Newcombe, and D. A. Compston. 1987. A double-blind controlled trial of high dose methylprednisolone in patients with multiple sclerosis: 1. Clinical effects. Journal of Neurology, Neurosurgery \& Psychiatry 50:511-516.

35. Beck, R. W., P. A. Cleary, J. D. Trobe, D. I. Kaufman, M. J. Kupersmith, D. W. Paty, C. H. Brown, and G. The optic neuritis study. 1993. The effect of corticosteroids for acute optic neuritis on the subsequent development of multiple sclerosis. New England Journal of Medicine 329:1764-1769.

36. Alfons, B., C. K. Bernd, and H. Hans-Peter. 2004. Biologic role of interferon beta in multiple sclerosis. Journal of Neurology V251:ii10-ii14.

37. Mitsdoerffer, M., B. Schreiner, B. C. Kieseier, O. Neuhaus, J. Dichgans, H.-P. Hartung, M. Weller, and H. Wiendl. 2005. Monocyte-derived HLA-G acts as a strong inhibitor of autologous CD4 T cell activation and is upregulated by interferon-[beta] in vitro and in vivo: rationale for the therapy of multiple sclerosis. Journal of Neuroimmunology 159:155-164.

38. Sharief, M. K., and R. Hentges. 1991. Association between tumor necrosis factoralpha and disease progression in patients with multiple sclerosis. New England Journal of Medicine 325:467-472. 
39. Titelbaum, D. S., A. Degenhardt, and R. P. Kinkel. 2005. Anti-tumor necrosis factor alpha-associated multiple sclerosis. American Journal of Neuroradiology 26:1548-1550.

40. Selmaj, K., W. Papierz, A. Glabinski, and T. Kohno. 1995. Prevention of chronic relapsing experimental autoimmune encephalomyelitis by soluble tumor necrosis factor receptor I. Journal of Neuroimmunology 56:135-141.

41. Liu, J., M. W. Marino, G. Wong, D. Grail, A. Dunn, J. Bettadapura, A. J. Slavin, L. Old, and C. C. Bernard. 1998. TNF is a potent anti-inflammatory cytokine in autoimmune-mediated demyelination. Nature Medicine 4:78-83.

42. Cannella, B., Y. L. Gao, C. Brosnan, and C. S. Raine. 1996. IL-10 fails to abrogate experimental autoimmune encephalomyelitis. Journal of Neuroscience Research 45:735-746.

43. Cua, D. J., B. Hutchins, D. M. LaFace, S. A. Stohlman, and R. L. Coffman. 2001. Central nervous system expression of IL-10 inhibits autoimmune encephalomyelitis. Journal of Immunology 166:602-608.

44. Chen, Y., V. K. Kuchroo, J. Inobe, D. A. Hafler, and H. L. Weiner. 1994. Regulatory T-cell clones induced by oral tolerance: suppression of autoimmune encephalomyelitis. Science 265:1237-1240.

45. Sloan-Lancaster, J., B. D. Evavold, and P. M. Allen. 1993. Induction of T-cell anergy by altered T-cell receptor ligand on live antigen presenting cells. Nature 363:156-159.

46. Brocke, S., K. Gijbels, M. Allegretta, I. Ferber, C. Piercy, T. Blankenstein, R. Martin, U. Utz, N. Karin, D. Mitchell, T. Veromaa, A. Waisman, A. Gaur, P. Conlon, N. Ling, P. J. Fairchild, D. C. Wraith, A. O'Garra, C. G. Fathman, and L. Steinman. 1996. Treatment of experimental encephalomyelitis with a peptide analogue of myelin basic protein. Nature 379:343-346.

47. Bielekova, B., B. Goodwin, N. Richert, I. Cortese, T. Kondo, G. Afshar, B. Gran, J. Eaton, J. Antel, J. A. Frank, H. F. McFarland, and R. Martin. 2000.

Encephalitogenic potential of the myelin basic protein peptide (amino acids 8399) in multiple sclerosis: results of a phase II clinical trial with an altered peptide ligand. Nature Medicine 6:1167-1175.

48. Duda, P. W., M. C. Schmied, S. L. Cook, J. I. Krieger, and D. A. Hafler. 2000. Glatiramer acetate (Copaxone) induces degenerate, Th2-polarized immune responses in patients with multiple sclerosis. Journal of Clinical Investigation 105:967-976.

49. Lando, Z., D. Teitelbaum, and R. Arnon. 1979. Effect of cyclophosphamide on suppressor cell activity in mice unresponsive to EAE. Journal of Immunology 123:2156-2160.

50. Aharoni, R., B. Kayhan, R. Eilam, M. Sela, and R. Arnon. 2003. Glatiramer acetate-specific T-cells in the brain express T helper $2 / 3$ cytokines and brainderived neurotrophic factor in situ. Proceedings of the National Academy of Sciences of the United States of America 100:14157-14162.

51. Johnson, K. P., B. R. Brooks, J. A. Cohen, C. C. Ford, J. Goldstein, R. P. Lisak, L. W. Myers, H. S. Panitch, J. W. Rose, and R. B. Schiffer. 1995. Copolymer 1 reduces relapse rate and improves disability in relapsing-remitting multiple 
sclerosis: results of a phase III multicenter, double-blind placebo-controlled trial. The Copolymer 1 multiple sclerosis study group. Neurology 45:1268-1276.

52. Weiner, H. L. 1997. Oral tolerance: immune mechanisms and treatment of autoimmune diseases. Immunology Today 18:335-343.

53. Zhang, J., R. Medaer, P. Stinissen, D. Hafler, and J. Raus. 1993. MHC-restricted depletion of human myelin basic protein-reactive T-cells by T-cell vaccination. Science 261:1451-1454.

54. Yednock, T. A., C. Cannon, L. C. Fritz, F. Sanchez-Madrid, L. Steinman, and N. Karin. 1992. Prevention of experimental autoimmune encephalomyelitis by antibodies against [alpha]4[beta]l integrin. Nature 356:63-66.

55. Hauser, S. L., and H. L. Weiner. 2006. Natalizumab: immune effects and implications for therapy. Annals of Neurology 59:731-732.

56. Martino, G., P. L. Poliani, R. Furlan, P. Marconi, J. C. Glorioso, L. Adorini, and G. Comi. 2000. Cytokine therapy in immune-mediated demyelinating diseases of the central nervous system: a novel gene therapy approach. Journal of Neuroimmunology 107:184-190.

57. Costa, G. L., J. M. Benson, C. M. Seroogy, P. Achacoso, C. G. Fathman, and G. P. Nolan. 2000. Targeting rare populations of murine antigen-specific $\mathrm{T}$ lymphocytes by retroviral transduction for potential application in gene therapy for autoimmune disease. Journal of Immunology 164:3581-3590.

58. Nguyen, P., and T. L. Geiger. 2003. Antigen-specific targeting of CD $8^{+}$T-cells with receptor-modified T lymphocytes. GeneTherapy 10:594-604.

59. Jyothi, M. D., R. A. Flavell, and T. L. Geiger. 2002. Targeting autoantigenspecific T-cells and suppression of autoimmune encephalomyelitis with receptormodified T lymphocytes. Nature Biotechnology 20:1215-1220.

60. Rossig, C., and M. K. Brenner. 2003. Chimeric T-cell receptors for the targeting of cancer cells. Acta Haematologica 110:154-159.

61. Goverman, J., S. M. Gomez, K. D. Segesman, T. Hunkapiller, W. E. Laug, and L. Hood. 1990. Chimeric immunoglobulin T-cell receptor proteins form functional receptors: implications for $\mathrm{T}$ cell receptor complex formation and activation. Cell 60:929-939.

62. Bird, R. E., K. D. Hardman, J. W. Jacobson, S. Johnson, B. M. Kaufman, S. M. Lee, T. Lee, S. H. Pope, G. S. Riordan, and M. Whitlow. 1988. Single-chain antigen-binding proteins. Science 242:423-426.

63. Eshhar, Z., T. Waks, G. Gross, and D. G. Schindler. 1993. Specific activation and targeting of cytotoxic lymphocytes through chimeric single chains consisting of antibody-binding domains and the gamma or zeta subunits of the immunoglobulin and T-cell receptors. Proceedings of the National Academy of Sciences of the United States of America 90:720-724.

64. Tan, L. K., R. J. Shopes, V. T. Oi, and S. L. Morrison. 1990. Influence of the hinge region on complement activation, $\mathrm{C} 1 \mathrm{q}$ binding, and segmental flexibility in chimeric human immunoglobulins. Proceedings of the National Academy of Sciences of the United States of America 87:162-166.

65. Moritz, D., and B. Groner. 1995. A spacer region between the single chain antibody and the CD3 zeta-chain domain of chimeric T-cell receptor components 
is required for efficient ligand binding and signaling activity. Gene Therapy 2:539-546.

66. Mueller, D. L., M. K. Jenkins, and R. H. Schwartz. 1989. Clonal expansion versus functional clonal inactivation: a costimulatory signalling pathway determines the outcome of T-cell antigen receptor occupancy. Annual Review of Immunology 7:445-480.

67. Krause, A., H. F. Guo, J. B. Latouche, C. Tan, N. K. Cheung, and M. Sadelain. 1998. Antigen-dependent CD28 signaling selectively enhances survival and proliferation in genetically modified activated human primary $\mathrm{T}$ lymphocytes. Journal of Experimental Medicine 188:619-626.

68. Hurtado, J. C., Y. J. Kim, and B. S. Kwon. 1997. Signals through 4-1BB are costimulatory to previously activated splenic T-cells and inhibit activationinduced cell death. Journal of Immunology 158:2600-2609.

69. Kootstra, N. A., and I. M. Verma. 2003. Gene therapy with viral vectors. Annual Review of Pharmacology and Toxicology 43:413-439.

70. Mitsuyasu, R. T., P. A. Anton, S. G. Deeks, D. T. Scadden, E. Connick, M. T. Downs, A. Bakker, M. R. Roberts, C. H. June, S. Jalali, A. A. Lin, R. PennathurDas, and K. M. Hege. 2000. Prolonged survival and tissue trafficking following adoptive transfer of CD4zeta gene-modified autologous $\mathrm{CD}^{+}$and $\mathrm{CD} 8^{+}$T-cells in human immunodeficiency virus-infected subjects. Blood 96:785-793.

71. Walker, R. E., C. M. Bechtel, V. Natarajan, M. Baseler, K. M. Hege, J. A. Metcalf, R. Stevens, A. Hazen, R. M. Blaese, C. C. Chen, S. F. Leitman, J. Palensky, J. Wittes, R. T. Davey, Jr., J. Falloon, M. A. Polis, J. A. Kovacs, D. F. Broad, B. L. Levine, M. R. Roberts, H. Masur, and H. C. Lane. 2000. Long-term in vivo survival of receptor-modified syngeneic T-cells in patients with human immunodeficiency virus infection. Blood 96:467-474.

72. Moritz, D., W. Wels, J. Mattern, and B. Groner. 1994. Cytotoxic T lymphocytes with a grafted recognition specificity for ERBB2-expressing tumor cells. Proceedings of the National Academy of Sciences of the United States of America 91:4318-4322.

73. Canevari, S., G. Stoter, F. Arienti, G. Bolis, M. I. Colnaghi, E. M. Di Re, A. M. Eggermont, S. H. Goey, J. W. Gratama, C. H. Lamers, and et al. 1995. Regression of advanced ovarian carcinoma by intraperitoneal treatment with autologous $\mathrm{T}$ lymphocytes retargeted by a bispecific monoclonal antibody. Journal of the National Cancer Institute 87:1463-1469.

74. Bleumer, I., A. Knuth, E. Oosterwijk, R. Hofmann, Z. Varga, C. Lamers, W. Kruit, S. Melchior, C. Mala, S. Ullrich, P. De Mulder, P. F. Mulders, and J. Beck. 2004. A phase II trial of chimeric monoclonal antibody G250 for advanced renal cell carcinoma patients. British Journal of Cancer 90:985-990.

75. Heslop, H. E., C. Y. Ng, C. Li, C. A. Smith, S. K. Loftin, R. A. Krance, M. K. Brenner, and C. M. Rooney. 1996. Long-term restoration of immunity against Epstein-Barr virus infection by adoptive transfer of gene-modified virus-specific T lymphocytes. Nature Medicine 2:551-555.

76. Geiger, T. L., P. Nguyen, D. Leitenberg, and R. A. Flavell. 2001. Integrated src kinase and costimulatory activity enhances signal transduction through singlechain chimeric receptors in T lymphocytes. Blood 98:2364-2371. 
77. Matloubian, M., R. J. Concepcion, and R. Ahmed. 1994. CD4+ T-cells are required to sustain $\mathrm{CD} 8+$ cytotoxic $\mathrm{T}$-cell responses during chronic viral infection. Journal of Virology 68:8056-8063.

78. Bonifacino, J. S., and L. M. Traub. 2003. Signals for sorting of transmembrane proteins to endosomes and lysosomes. Annual Review of Biochemistry 72:395447.

79. Letourneur, F., and R. D. Klausner. 1992. A novel di-leucine motif and a tyrosinebased motif independently mediate lysosomal targeting and endocytosis of CD3 chains. Cell 69:1143-1157.

80. Johnson, K. F., and S. Kornfeld. 1992. A His-Leu-Leu sequence near the carboxyl terminus of the cytoplasmic domain of the cation-dependent mannose 6phosphate receptor is necessary for the lysosomal enzyme sorting function. The Journal of Biological Chemistry 267:17110-17115.

81. Dell'Angelica, E. C., R. Puertollano, C. Mullins, R. C. Aguilar, J. D. Vargas, L. M. Hartnell, and J. S. Bonifacino. 2000. GGAs: A family of ADP ribosylation factor-binding proteins related to adaptors and associated with the Golgi complex. Journal of Cell Biology 149:81-94.

82. Kato, Y., S. Misra, R. Puertollano, J. H. Hurley, and J. S. Bonifacino. 2002. Phosphoregulation of sorting signal-VHS domain interactions by a direct electrostatic mechanism. Nature Structural Biology 9:532-536.

83. Doray, B., K. Bruns, P. Ghosh, and S. A. Kornfeld. 2002. Autoinhibition of the ligand-binding site of GGA1/3 VHS domains by an internal acidic clusterdileucine motif. Proceedings of the National Academy of Sciences of the United States of America 99:8072-8077.

84. Finney, H. M., A. D. G. Lawson, C. R. Bebbington, and A. N. C. Weir. 1998. Chimeric receptors providing both primary and costimulatory signaling in T-cells from a single gene product. Journal of Immunology 161:2791-2797.

85. Kreitman, R. J., W. H. Wilson, J. D. White, M. Stetler-Stevenson, E. S. Jaffe, S. Giardina, T. A. Waldmann, and I. Pastan. 2000. Phase I trial of recombinant immunotoxin anti-Tac(Fv)-PE38 (LMB-2) in patients with hematologic malignancies. Journal of Clinical Oncology 18:1622-1636.

86. Wucherpfennig, K. W., A. Sette, S. Southwood, C. Oseroff, M. Matsui, J. L. Strominger, and D. A. Hafler. 1994. Structural requirements for binding of an immunodominant myelin basic protein peptide to DR2 isotypes and for its recognition by human T-cell clones. Journal of Experimental Medicine 179:279290.

87. Mycko, M. P., H. Waldner, D. E. Anderson, K. D. Bourcier, K. W. Wucherpfennig, V. K. Kuchroo, and D. A. Hafler. 2004. Cross-reactive TCR responses to self antigens presented by different MHC class II molecules. Journal of Immunology 173:1689-1698.

88. Madsen, L. S., E. C. Andersson, L. Jansson, M. Krogsgaard, C. B. Andersen, J. Engberg, J. L. Strominger, A. Svejgaard, J. P. Hjorth, R. Holmdahl, K. W. Wucherpfennig, and L. Fugger. 1999. A humanized model for multiple sclerosis using HLA-DR2 and a human T-cell receptor. Nature Genetics 23:343-347. 
89. de Felipe, P., V. Martin, M. L. Cortes, M. Ryan, and M. Izquierdo. 1999. Use of the $2 \mathrm{~A}$ sequence from foot-and-mouth disease virus in the generation of retroviral vectors for gene therapy. Gene Therapy 6:198-208.

90. Kozak, M. 1986. Point mutations define a sequence flanking the AUG initiator codon that modulates translation by eukaryotic ribosomes. Cell 44:283-292.

91. de Felipe, P., G. A. Luke, L. E. Hughes, D. Gani, C. Halpin, and M. D. Ryan. 2006. E unum pluribus: multiple proteins from a self-processing polyprotein. Trends in Biotechnology 24:68-75.

92. Heemskerk, M. H., M. W. Schilham, H. M. Schoemaker, G. Spierenburg, W. J. Spaan, and C. J. Boog. 1995. Activation of virus-specific major histocompatibility complex class II-restricted $\mathrm{CD}^{+}$cytotoxic T-cells in CD4-deficient mice.

European Journal of Immunology 1109-1112.

93. Steinman, L., R. Martin, C. Bernard, P. Conlon, and J. R. Oksenberg. 2002. Multiple sclerosis: deeper understanding of its pathogenesis reveals new targets for therapy. Annual Review of Neuroscience 25:491-505.

94. Ota, K., M. Matsui, E. L. Milford, G. A. Mackin, H. L. Weiner, and D. A. Hafler. 1990. T-cell recognition of an immunodominant myelin basic protein epitope in multiple sclerosis. Nature 346:183-187.

95. van Oers, N. S. C., P. E. Love, E. W. Shores, and A. Weiss. 1998. Regulation of TCR signal transduction in murine thymocytes by multiple TCR \{zeta\}-chain signaling motifs. Journal of Immunology 160:163-170.

96. Vignali, D. A., J. Moreno, D. Schiller, and G. J. Hammerling. 1992. Speciesspecific binding of CD4 to the beta 2 domain of major histocompatibility complex class II molecules. Journal of Experimental Medicine 175:925-932.

97. Lombardi, G., L. Barber, G. Aichinger, T. Heaton, S. Sidhu, J. R. Batchelor, and R. I. Lechler. 1991. Structural analysis of anti-DR1 allorecognition by using DR1/H-2Ek hybrid molecules. Influence of the beta 2-domain correlates with CD4 dependence. Journal of Immunology 147:2034-2040.

98. Woods, A., H. Y. Chen, M. E. Trumbauer, A. Sirotina, R. Cummings, and D. M. Zaller. 1994. Human major histocompatibility complex class II-restricted T-cell responses in transgenic mice. Journal of Experimental Medicine 180:173-181.

99. Altmann, D. M., D. C. Douek, A. J. Frater, C. M. Hetherington, H. Inoko, and J. I. Elliott. 1995. The T-cell response of HLA-DR transgenic mice to human myelin basic protein and other antigens in the presence and absence of human CD4. Journal of Experimental Medicine 181:867-875.

100. Zamvil, S. S., P. A. Nelson, D. J. Mitchell, R. L. Knobler, R. B. Fritz, and L. Steinman. 1985. Encephalitogenic T-cell clones specific for myelin basic protein. An unusual bias in antigen recognition. Journal of Experimental Medicine 162:2107-2124.

101. Lindsey, J. W., S. Hodgkinson, R. Mehta, D. Mitchell, D. Enzmann, and L. Steinman. 1994. Repeated treatment with chimeric anti-CD4 antibody in multiple sclerosis. Annals of Neurology 36:183-189.

102. Huseby, E. S., D. Liggitt, T. Brabb, B. Schnabel, C. Ohlen, and J. Goverman. 2001. A pathogenic role for myelin-specific $\mathrm{CD}^{+} \mathrm{T}$-cells in a model for multiple sclerosis. Journal of Experimental Medicine 194:669-676. 
103. Vanderlugt, C. L., and S. D. Miller. 2002. Epitope spreading in immune-mediated diseases: implications for immunotherapy. Nature Reviews 2:85-95.

104. Greter, M., F. L. Heppner, M. P. Lemos, B. M. Odermatt, N. Goebels, T. Laufer, R. J. Noelle, and B. Becher. 2005. Dendritic cells permit immune invasion of the CNS in an animal model of multiple sclerosis. Nature Medicine 11:328-334.

105. McMahon, E. J., S. L. Bailey, C. V. Castenada, H. Waldner, and S. D. Miller. 2005. Epitope spreading initiates in the CNS in two mouse models of multiple sclerosis. Nature Medicine 11:335-339.

106. Tuohy, V. K., M. Yu, L. Yin, P. M. Mathisen, J. M. Johnson, and J. A. Kawczak. 2000. Modulation of the IL-10/IL-12 cytokine circuit by interferon-beta inhibits the development of epitope spreading and disease progression in murine autoimmune encephalomyelitis. Journal of Neuroimmunology 111:55-63.

107. Imai, C., and D. Campana. 2004. Genetic modification of T-cells for cancer therapy. Journal of Biological Regulators and Homeostatic Agents 18:62-71.

108. Marktel, S., Z. Magnani, F. Ciceri, S. Cazzaniga, S. R. Riddell, C. Traversari, C. Bordignon, and C. Bonini. 2003. Immunologic potential of donor lymphocytes expressing a suicide gene for early immune reconstitution after hematopoietic Tcell-depleted stem cell transplantation. Blood 101:1290-1298. 


\section{VITA}

Ioana Moisini was born in Iasi, Romania. She attended "Emil Racovita" high school and from September 1988 to July 1994 she was a medical student at the University of Medicine and Pharmacy "Gr. T. Popa” in Iasi, Romania. She graduated with a 9.57 average (on a scale of 1 to 10) and pursued a clinical residency in Pathology

from 1995 to 1999. She passed the Pathology Board exam in November 1999 and worked as an attending at "Socola Psychiatric Hospital" until 2001. In August 2001 Ioana was enrolled as a graduate student in the department of Pathology at University of Tennessee Health Science Center and began working on autoimmune conditions in the laboratory of Dr. Terrence Geiger at St. Jude Children's Research Hospital. She defended her doctoral dissertation in June 2007 and will graduate as Doctor of Philosophy in December 2007. Ioana Moisini has received a postdoctoral fellowship at Albert Einstein University in New York and will continue working on autoimmune conditions at North Shore Jewish Long Island Medical Center/Feinstein Institute, New York. 